\title{
ON SCHMIDT AND SUMMERER PARAMETRIC GEOMETRY OF NUMBERS
}

\author{
DAMIEN ROY
}

\begin{abstract}
Recently, W. M. Schmidt and L. Summerer introduced a new theory which allowed them to recover the main known inequalities relating the usual exponents of Diophantine approximation to a point in $\mathbb{R}^{n}$, and to discover new ones. They first note that these exponents can be computed in terms of the successive minima of a parametric family of convex bodies attached to the given point. Then they prove that the $n$-tuple of these successive minima can in turn be approximated up to bounded difference by a function from a certain class. In this paper, we show that the same is true within a smaller and simpler class of functions which we call rigid systems. We also show that conversely, given a rigid system, there exists a point in $\mathbb{R}^{n}$ whose associated family of convex bodies has successive minima which approximate that rigid system up to bounded difference. As a consequence, the problem of describing the joint spectrum of a family of exponents of Diophantine approximation is reduced to combinatorial analysis.
\end{abstract}

\section{INTRODUCTION}

In two recent outstanding papers [13] and [14, W. M. Schmidt and L. Summerer study the joint behavior of the $n$ successive minima of certain one parameter families of convex bodies in $\mathbb{R}^{n}$, as a function of the parameter. Then, they show how their results can be used to recover important inequalities relating standard exponents of Diophantine approximation attached to points in $\mathbb{R}^{n}$, and they find new ones. The goal of this paper is to simplify and to complete some aspects of their theory. It also aims at promoting their wonderful idea which, the author is convinced, will have a major impact in Diophantine approximation as it provides a new simple way of thinking about problems of simultaneous approximation.

Let $n \geq 2$ be an integer, let $\mathbf{x} \cdot \mathbf{y}$ denote the standard scalar product of vectors $\mathbf{x}, \mathbf{y} \in \mathbb{R}^{n}$, and let $\|\mathbf{x}\|=(\mathbf{x} \cdot \mathbf{x})^{1 / 2}$ denote the Euclidean norm of $\mathbf{x}$. Up to re-scaling, the families of convex bodies considered by Schmidt and Summerer are dual to

$$
\mathcal{C}_{\mathbf{u}}\left(e^{q}\right):=\left\{\mathbf{x} \in \mathbb{R}^{n} ;\|\mathbf{x}\| \leq 1,|\mathbf{x} \cdot \mathbf{u}| \leq e^{-q}\right\} \quad(q \geq 0),
$$

where $\mathbf{u}$ is a given unit vector in $\mathbb{R}^{n}$. However, working with these alternative families brings little difference and, for our purpose, is more convenient. For each $j=1, \ldots, n$ and each $q \geq 0$, we denote by $L_{\mathbf{u}, j}(q)$ the smallest real number $L \geq 0$ such that $e^{L} \mathcal{C}_{\mathbf{u}}\left(e^{q}\right)$ contains at least $j$ linearly independent points of $\mathbb{Z}^{n}$. Then, we group these successive minima into a single map $\mathbf{L}_{\mathbf{u}}:[0, \infty) \rightarrow \mathbb{R}^{n}$ by setting

$$
\mathbf{L}_{\mathbf{u}}(q)=\left(L_{\mathbf{u}, 1}(q), \ldots, L_{\mathbf{u}, n}(q)\right) \quad(q \geq 0) .
$$

2000 Mathematics Subject Classification. Primary 11J13; Secondary 11J82.

Work partially supported by NSERC. 
Schmidt and Summerer establish many properties of this map. We will recall them in the next section. Here we simply mention that each component $L_{\mathbf{u}, j}:[0, \infty) \rightarrow \mathbb{R}$ is continuous and piecewise linear with slopes 0 and 1 , which means that its right derivative is 0 or 1 at each point $q \geq 0$, and the same for its left derivative at each point $q>0$. Equivalently, the graph of $L_{\mathbf{u}, j}$ is a connected polygon composed of line segments of slopes 0 and 1 . We also have $0 \leq L_{\mathbf{u}, 1}(q) \leq \cdots \leq L_{\mathbf{u}, n}(q)$ for each $q \geq 0$, which means that $\mathbf{L}_{\mathbf{u}}$ takes values in the set

$$
\Delta_{n}=\left\{\left(x_{1}, \ldots, x_{n}\right) \in \mathbb{R}^{n} ; x_{1} \leq \cdots \leq x_{n}\right\}
$$

of monotone increasing $n$-tuples of real numbers.

Schmidt and Summerer show that each function $\mathbf{L}_{\mathbf{u}}$ can be approximated up to bounded difference by functions from a certain class (see in the next section). One of our main results is that the same property holds within a simpler class. To describe it, we follow [14, §3], and define the combined graph of a set of real valued functions on an interval $I$ to be the union of their graphs in $I \times \mathbb{R}$. For a function $\mathbf{P}=\left(P_{1}, \ldots, P_{n}\right):[c, \infty) \rightarrow \Delta_{n}$, and a subinterval $I$ of $[c, \infty)$, we define the combined graph of $\mathbf{P}$ above $I$ to be the combined graph of its components $P_{1}, \ldots, P_{n}$ restricted to $I$. If $P$ is continuous and if the real numbers $q \geq c$ at which $P_{1}(q), \ldots, P_{n}(q)$ are not all distinct form a discrete subset of $[c, \infty)$, then the map $\mathbf{P}$ is uniquely determined by its combined graph over the full interval $[c, \infty)$. We also denote by $\Phi_{n}: \mathbb{R}^{n} \rightarrow \Delta_{n}$ the continuous map which lists the coordinates of a point in monotone increasing order. We can now introduce our basic combinatorial object.

Definition 1.1. Let $\delta \in(0, \infty)$ and let $s \in \mathbb{N}^{*} \cup\{\infty\}=\{\infty, 1,2,3, \ldots\}$. A canvas with mesh $\delta$ and cardinality $s$ in $\mathbb{R}^{n}$ is a triple consisting of a sequence of points $\left(\mathbf{a}^{(i)}\right)_{0 \leq i<s}$ in $\Delta_{n}$ together with two sequences of integers $\left(k_{i}\right)_{0 \leq i<s}$ and $\left(\ell_{i}\right)_{0 \leq i<s}$ of the same cardinality $s$ such that, for each index $i$ with $0 \leq i<s$,

(C1) the coordinates $\left(a_{1}^{(i)}, \ldots, a_{n}^{(i)}\right)$ of $\mathbf{a}^{(i)}$ form a strictly increasing sequence of positive integer multiples of $\delta$,

(C2) we have $1 \leq k_{0} \leq \ell_{0}=n$ and $1 \leq k_{i}<\ell_{i} \leq n$ if $i \geq 1$,

(C3) if $i+1<s$, then $k_{i} \leq \ell_{i+1}, a_{\ell_{i+1}}^{(i)}+\delta \leq a_{\ell_{i+1}}^{(i+1)}$ and

$$
\left(a_{1}^{(i)}, \ldots, \widehat{a_{k_{i}}^{(i)}}, \ldots, a_{n}^{(i)}\right)=\left(a_{1}^{(i+1)}, \ldots, \widehat{a_{\ell_{i+1}}^{(i+1)}}, \ldots, a_{n}^{(i+1)}\right)
$$

where the hat on a coordinate means that it is omitted.

Thus, in such a sequence $\left(\mathbf{a}^{(i)}\right)_{0 \leq i<s}$, each point $\mathbf{a}^{(i+1)}$ with $i+1<s$ is obtained from the preceding point $\mathbf{a}^{(i)}$ by replacing one of its coordinates by a larger multiple of $\delta$, different from all other coordinates of $\mathbf{a}^{(i)}$, and then by re-ordering the new $n$-tuple. In particular, this sequence uniquely determines the sequence $\left(k_{i}\right)_{0 \leq i<s}$ up to its last term $k_{s-1}$ if $s<\infty$, and the full sequence $\left(\ell_{i}\right)_{0 \leq i<s}$ since $\ell_{0}=n$. When $s$ is finite, we also define $\ell_{s}=n$ and

$$
\mathbf{a}^{(s)}=\left(a_{1}^{(s)}, \ldots, a_{n-1}^{(s)}, \infty\right) \in \mathbb{R}^{n-1} \times\{\infty\}
$$

so that (C3) holds for $i=s-1$. 
Definition 1.2. To each canvas of mesh $\delta>0$ as in Definition 1.1, we associate the function $\mathbf{P}:\left[q_{0}, \infty\right) \rightarrow \Delta_{n}$ given by

$$
\mathbf{P}(q)=\Phi_{n}\left(a_{1}^{(i)}, \ldots, \widehat{a_{k_{i}}^{(i)}}, \ldots, a_{n}^{(i)}, a_{k_{i}}^{(i)}+q-q_{i}\right) \quad\left(0 \leq i<s, q_{i} \leq q<q_{i+1}\right),
$$

where $q_{i}=a_{1}^{(i)}+\cdots+a_{n}^{(i)}(0 \leq i<s)$ and $q_{s}=\infty$ if $s<\infty$. We say that such a function is a rigid $n$-system with mesh $\delta$ and that $\left(q_{i}\right)_{0 \leq i<s}$ is its sequence of switch numbers.

Since $a_{k_{i}}^{(i)}+q_{i+1}-q_{i}=a_{\ell_{i+1}}^{(i+1)}$ when $i+1<s$, such a map $\mathbf{P}$ is continuous. Its combined graph over an interval $\left[q_{i}, q_{i+1}\right)$ with $0 \leq i<s$ consists of $n-1$ horizontal half-open line segments and one half-open line segment of slope 1 . Their left end-points are the points $\left(q_{i}, a_{j}^{(i)}\right)(1 \leq j \leq n)$ and, if $i+1<s$, their right end-points are $\left(q_{i+1}, a_{j}^{(i+1)}\right)(1 \leq j \leq n)$. In this context, the condition $(\mathrm{C} 2)$, imposed on the canvas, translates into the fact that, for each index $i$ with $1 \leq i<s$, the straight line extending the line segment of slope 1 over $\left[q_{i}, q_{i+1}\right)$ lies to the right of the straight line which extends the line segment of slope 1 over the preceding interval $\left[q_{i-1}, q_{i}\right)$. Figure 1 illustrates this by showing the combined graph of a rigid 5-system with mesh 1 attached to the canvas $\{(1,2,4,5,8),(1,2,4,7,8),(1,4,5,7,8)\}$ of cardinality $s=3$ with $k_{2}=1$.

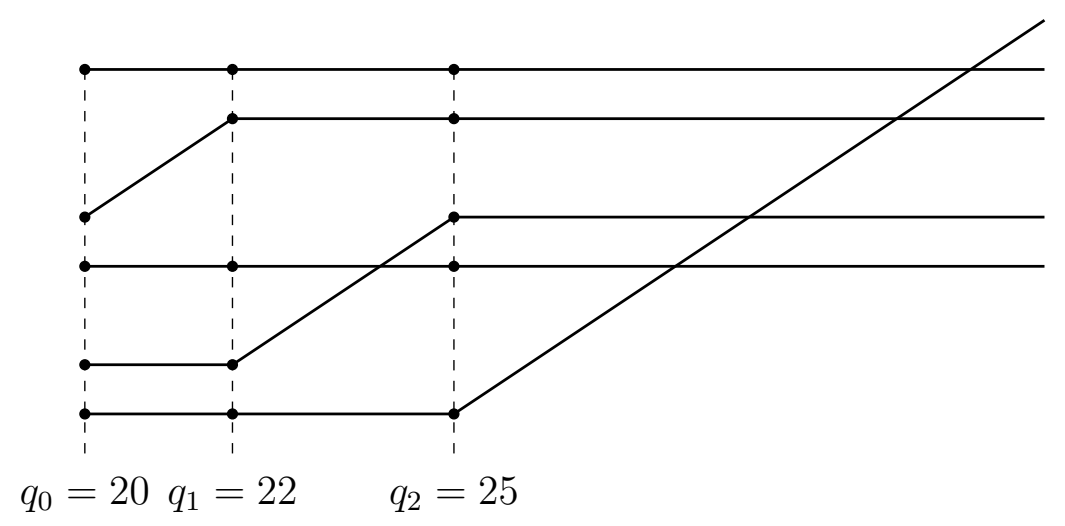

FiguRE 1. The combined graph of a rigid 5-system.

The main goal of this paper is to prove the following result.

Theorem 1.3. Let $n \geq 2$ be an integer and let $\delta \in(0, \infty)$. For each unit vector $\mathbf{u}$ of $\mathbb{R}^{n}$, there exists a rigid system $\mathbf{P}:\left[q_{0}, \infty\right) \rightarrow \Delta_{n}$ with mesh $\delta$ such that $\mathbf{L}_{\mathbf{u}}-\mathbf{P}$ is bounded on $\left[q_{0}, \infty\right)$. Conversely, for each rigid system $\mathbf{P}:\left[q_{0}, \infty\right) \rightarrow \Delta_{n}$ with mesh $\delta$, there exists a unit vector $\mathbf{u}$ in $\mathbb{R}^{n}$ such that $\mathbf{L}_{\mathbf{u}}-\mathbf{P}$ is bounded on $\left[q_{0}, \infty\right)$.

To mention but one application, recall that, to each unit vector $\mathbf{u}$ in $\mathbb{R}^{n}$, one attaches several exponents of Diophantine approximation which measure how well $\mathbf{u}$ can be approximated by rational vector subspaces of $\mathbb{R}^{n}$ of a given dimension $d$ (see [1, 8, 12]). For $d=n-1$, one uses $\tau(\mathbf{u})$, respectively $\hat{\tau}(\mathbf{u})$, defined as the supremum of all real numbers $\tau>0$ for which the system of inequalities

$$
\|\mathbf{x}\| \leq X \quad \text { and } \quad|\mathbf{x} \cdot \mathbf{u}| \leq X^{-\tau}
$$


admits a non-zero solution $\mathbf{x}$ in $\mathbb{Z}^{n}$ for arbitrarily large values of $X$, respectively for all sufficiently large values of $X$. For dimension $d=1$, one uses the dual exponents $\lambda(\mathbf{u})$, respectively $\hat{\lambda}(\mathbf{u})$, defined as the supremum of all $\lambda>0$ such that

$$
\|\mathbf{x}\| \leq X \quad \text { and } \quad\|\mathbf{x} \wedge \mathbf{u}\| \leq X^{-\lambda}
$$

admits a non-zero solution $\mathbf{x}$ in $\mathbb{Z}^{n}$ for arbitrarily large values of $X$, respectively for all sufficiently large values of $X$, where $\|\mathbf{x} \wedge \mathbf{u}\|$ represents the norm of $\mathbf{x} \wedge \mathbf{u}$ for the Euclidean structure of $\bigwedge^{2} \mathbb{R}^{n}$ inherited from $\mathbb{R}^{n}$ (see the next section). With this notation, Theorem 1.3 admits the following consequence.

Corollary 1.4. Let $n \in \mathbb{N}$ with $n \geq 2$ and let $\delta>0$. The map $\theta: \mathbb{R}^{4} \rightarrow \mathbb{R}^{4}$ given by

$$
\theta(\tau, \hat{\tau}, \hat{\lambda}, \lambda)=\left(\frac{1}{\tau+1}, \frac{1}{\hat{\tau}+1}, \frac{\hat{\lambda}}{\hat{\lambda}+1}, \frac{\lambda}{\lambda+1}\right)
$$

establishes a bijection between the set of quadruples $(\tau(\mathbf{u}), \hat{\tau}(\mathbf{u}), \hat{\lambda}(\mathbf{u}), \lambda(\mathbf{u}))$ where $\mathbf{u}$ runs through all unit vectors of $\mathbb{R}^{n}$ with $\mathbb{Q}$-linearly independent coordinates, and the set of quadruples

$$
\left(\liminf _{q \rightarrow \infty} \frac{P_{1}(q)}{q}, \limsup _{q \rightarrow \infty} \frac{P_{1}(q)}{q}, \liminf _{q \rightarrow \infty} \frac{P_{n}(q)}{q}, \limsup _{q \rightarrow \infty} \frac{P_{n}(q)}{q}\right)
$$

where $\mathbf{P}=\left(P_{1}, \ldots, P_{n}\right)$ runs through all rigid n-systems with mesh $\delta$ for which $P_{1}$ is unbounded.

The proof is clear based on the general philosophy developed by Schmidt and Summerer in their above-mentioned papers. Namely, if a unit vector $\mathbf{u}$ and a rigid $n$-system $\mathbf{P}=$ $\left(P_{1}, \ldots, P_{n}\right)$ with mesh $\delta$ are such that the difference $\mathbf{L}_{\mathbf{u}}-\mathbf{P}$ is bounded, then

$$
\liminf _{q \rightarrow \infty} \frac{P_{1}(q)}{q}=\liminf _{q \rightarrow \infty} \frac{L_{\mathbf{u}, 1}(q)}{q}=\frac{1}{\tau(\mathbf{u})+1}
$$

with similar equalities for the three other components of the quadruple (1.1). Moreover, in that case, $\mathbf{u}$ has $\mathbb{Q}$-linearly independent coordinates if and only if $P_{1}$ is unbounded.

The above corollary could easily be extended to deal with all the intermediate exponents of Schmidt and Laurent [8, 12, thereby solving a conjecture of Schmidt and Summerer in [14, $\S 4]$. In the present context, the latter authors show that $\theta$ maps injectively the quadruples $(\tau(\mathbf{u}), \hat{\tau}(\mathbf{u}), \hat{\lambda}(\mathbf{u}), \lambda(\mathbf{u}))$ to those of the form (1.1) where $\mathbf{P}$ runs through the larger set of what they call proper $(n, \gamma)$-systems (see Section 2.5 below). From this, they recover the celebrated Khintchine's and Jarník's transference principles [4, 5, 6] as well as more recent results of Bugeaud, Laurent and Moshchevitin from [1, 7, 8, 10]. They also prove new results [13, 14, 15]. Recently, Laurent gave in [7] a complete description of the joint spectrum of $(\tau, \hat{\tau}, \hat{\lambda}, \lambda)$ in dimension $n=3$. For larger dimension $n \geq 4$, the problem is open and the above corollary reduces it to combinatorial analysis. Note however that the present study does not apply to the more general exponents introduced by German in [2] (see also [12]).

In the next section, we recast in our setting the result of Schmidt and Summerer which approximates the maps $\mathbf{L}_{\mathbf{u}}$ by $(n, \gamma)$-systems. We also present there the intermediate results of geometry of number which are involved in the proof. In Section 3, we use the same 
results to analyze the situation where a rigid system with large mesh is, in comparison, well approximated by the map $\mathbf{L}_{\mathbf{u}}$ for some unit vector $\mathbf{u}$. The results that we obtain there complement Theorem [1.3. They also motivate the constructions of Section 5 by which we prove the second assertion of Theorem 1.3 for rigid systems with sufficiently large mesh (cf. [11] for a special case of this construction). The last piece of the puzzle is provided by Sections 6 and 7 which construct an approximation to an arbitrary $(n, \gamma)$-system by a rigid system with given sufficiently large mesh. The process is first to modify the $(n, \gamma)$-system to make what we call a reduced system (Section 6), then to approximate the resulting system by a step function and finally to construct a canvas out of this data (Section 7). The proof of our main Theorem 1.3 follows in Section 8. The reader may go there directly to get a precise idea on the role of all intermediate results.

\section{The theory of Schmidt And Summerer}

We start by recalling the basic notions and results from geometry of numbers that we will need throughout this paper. In few places, we provide a short argument in order to be able to specify the constants involved. We also present the central result of Schmidt and Summerer theory and its proof, which we adapt to our slightly different (dual) context. We hope that this will help the reader firstly because this makes our account relatively self contained, and secondly because, in the next section, we use the same notions and intermediate results to gather information about the inverse problem raised by this theorem. All results stated below are thus either classical or due to Schmidt and Summerer.

Let $V$ be a real Euclidean vector space of finite dimension $N \geq 1$. We use the following standard terminology (see [3]). By a convex body of $V$, we mean a compact convex neighborhood of 0 , stable under multiplication by -1 . By a lattice $\Lambda$ of $V$, we mean a discrete subgroup of $V$ of rank $N$. Its co-volume is the volume of the parallelepiped spanned by a basis of $\Lambda$ or, equivalently, the volume of $V / \Lambda$.

Suppose that $\mathcal{C}$ is a convex body of $V$, and $\Lambda$ a lattice of $V$. For each $j=1, \ldots, N$, we define the $j$-th minimum of $\mathcal{C}$ with respect to $\Lambda$, denoted $\lambda_{j}(\mathcal{C})$, to be the smallest real number $\lambda>0$ such that $\lambda \mathcal{C}$ contains at least $j$ elements of $\Lambda$ which are linearly independent over $\mathbb{R}$. Although this notation does not refer to the lattice $\Lambda$, this should not cause any ambiguity since, in all situations that we consider, the underlying lattice will be clear from the context. For each $\mathbf{x} \in V$, we further define $\lambda_{\mathbf{x}}(\mathcal{C})$, also denoted $\lambda(\mathbf{x}, \mathcal{C})$, to be the smallest real number $\lambda \geq 0$ such that $\mathbf{x} \in \lambda \mathcal{C}$. Then, there exist elements $\mathbf{x}_{1}, \ldots, \mathbf{x}_{N}$ of $\Lambda$ which are linearly independent over $\mathbb{R}$ and satisfy $\lambda\left(\mathbf{x}_{j}, \mathcal{C}\right)=\lambda_{j}(\mathcal{C})$ for $j=1, \ldots, N$. The function from $V$ to $\mathbb{R}$ mapping a point $\mathbf{x} \in V$ to $\lambda_{\mathbf{x}}(\mathcal{C})$ is called the distance function of $\mathcal{C}$. The notation $\lambda_{\mathbf{x}}(\mathcal{C})$ with the point $\mathbf{x}$ in index stresses the fact that, in the theory of Schmidt and Summerer, the convex body $\mathcal{C}$ is varying.

2.1. A general family of convex bodies. Let $V$ be as above and let $\Lambda$ be a lattice of $V$ with co-volume 1 . We choose a decomposition of $V$ into an orthogonal sum $V=U \perp W$ of two vector subspaces $U$ and $W$ with $W \neq 0$, and put $K=\operatorname{dim}_{\mathbb{R}}(W)$. Motivated by [14], we 
consider the family of convex bodies of $V$ given by

$$
\mathcal{C}(Q):=\left\{\mathbf{x} \in V ;\|\mathbf{x}\| \leq 1 \text { and }\left\|\operatorname{proj}_{W}(\mathbf{x})\right\| \leq Q^{-1}\right\} \quad(Q \geq 1),
$$

where $\operatorname{proj}_{W}$ stands for the orthogonal projection on $W$. For each $j=1, \ldots, N$, we define a function $L_{j}:[0, \infty) \rightarrow \mathbb{R}$ by

$$
L_{j}(q)=\log \lambda_{j}\left(\mathcal{C}\left(e^{q}\right)\right) \quad(q \geq 0) .
$$

Clearly, we have $L_{1}(q) \leq \cdots \leq L_{N}(q)$ for each $q \geq 0$, and so we get a map $\mathbf{L}:[0, \infty) \rightarrow \Delta_{N}$ by putting

$$
\mathbf{L}(q)=\left(L_{1}(q), \ldots, L_{N}(q)\right) \quad(q \geq 0) .
$$

Then, Minkowski's second convex body theorem yields the following result.

Lemma 2.1. For each $q \geq 0$, we have $\left|L_{1}(q)+\cdots+L_{N}(q)-K q\right| \leq N \log (N)$.

Proof. Let $Q \geq 1$. According to Minkowski's second convex body theorem, we have

$$
2^{N} / N ! \leq \lambda_{1}(\mathcal{C}(Q)) \cdots \lambda_{N}(\mathcal{C}(Q)) \operatorname{vol}(\mathcal{C}(Q)) \leq 2^{N} .
$$

To estimate the volume of $\mathcal{C}(Q)$, we choose an orthonormal basis $\left(\mathbf{e}_{1}, \ldots, \mathbf{e}_{K}\right)$ of $W$, extend it to an orthonormal basis $\left(\mathbf{e}_{1}, \ldots, \mathbf{e}_{N}\right)$ of $V$, and form the parallelepiped $P$ given by

$$
P=\left\{\mathbf{x} \in V ; \max _{1 \leq j \leq K}\left|\mathbf{x} \cdot \mathbf{e}_{j}\right| \leq Q^{-1} \text { and } \max _{K<j \leq N}\left|\mathbf{x} \cdot \mathbf{e}_{j}\right| \leq 1\right\} .
$$

Since $N^{-1} P \subset \mathcal{C}(Q) \subset P$, we obtain $(2 / N)^{N} Q^{-K} \leq \operatorname{vol}(\mathcal{C}(Q)) \leq 2^{N} Q^{-K}$. Thus

$$
(N !)^{-1} \leq \lambda_{1}(\mathcal{C}(Q)) \cdots \lambda_{N}(\mathcal{C}(Q)) Q^{-K} \leq N^{N}
$$

and the conclusion follows by taking logarithms.

2.2. Trajectories of points and combined graphs. Let the notation be as in $\$ 2.1$. For each $\mathbf{x} \in V$ and each $Q \geq 1$, we find

$$
\lambda_{\mathbf{x}}(\mathcal{C}(Q))=\lambda(\mathbf{x}, \mathcal{C}(Q))=\max \left\{\|\mathbf{x}\|, Q\left\|\operatorname{proj}_{W}(\mathbf{x})\right\|\right\} .
$$

When $\mathbf{x} \neq 0$, this number is positive, and so we obtain a function $L_{\mathbf{x}}:[0, \infty) \rightarrow \mathbb{R}$ by putting

$$
L_{\mathbf{x}}(q):=\log \lambda_{\mathbf{x}}\left(\mathcal{C}\left(e^{q}\right)\right)=\max \left\{\log \|\mathbf{x}\|, q+\log \left\|\operatorname{proj}_{W}(\mathbf{x})\right\|\right\} \quad(q \geq 0) .
$$

It is continuous and piecewise linear. If $\operatorname{proj}_{W}(\mathbf{x})=0$, it is constant equal to $\log \|\mathbf{x}\|$. Otherwise, it has slope 0 and then 1 . We also note that, if $\mathbf{x}$ and $\mathbf{y}$ are linearly dependent non-zero elements of $V$, then $L_{\mathbf{x}}$ and $L_{\mathbf{y}}$ differ by a constant. In particular, they have the same derivative $L_{\mathbf{x}}^{\prime}(q)=L_{\mathbf{y}}^{\prime}(q)$ at each point $q>0$ at which they are differentiable.

For a fixed non-zero $\mathrm{x}$ in $V$, the function $L_{\mathbf{x}}$ describes the position of $\mathbf{x}$ with respect to the varying family of convex bodies $\mathcal{C}\left(e^{q}\right)$. For this reason, we call its graph the trajectory of $\mathbf{x}$. Explicitly, this is the set $\left\{\left(q, L_{\mathbf{x}}(q)\right) ; 0 \leq q\right\}$. The inclusion

$$
\left\{\left(q, L_{j}(q)\right) ; 0 \leq q, 1 \leq j \leq N\right\} \subseteq\left\{\left(q, L_{\mathbf{x}}(q)\right) ; 0 \leq q, \mathbf{x} \in \Lambda \backslash\{0\}\right\}
$$

may thus be expressed by saying that the combined graph of $L_{1}, \ldots, L_{n}$ is contained in the combined graph of the functions $L_{\mathbf{x}}$ with $\mathbf{x} \in \Lambda \backslash\{0\}$, namely the union of the trajectories of these points. The goal is, in a sense, to compare these two sets. Clearly, we have

$$
L_{1}(q)=\inf \left\{L_{\mathbf{x}}(q) ; \mathbf{x} \in \Lambda \backslash\{0\}\right\} \quad(q \geq 0) .
$$


The next crucial result, due to Schmidt and Summerer, goes a step further.

Lemma 2.2. The functions $L_{1}, \ldots, L_{N}$ are continuous and piecewise linear with slopes 0 and 1 . At each point $q>0$ where $L_{1}$ changes slope from 1 to 0 , we have $L_{1}(q)=L_{2}(q)$.

Proof. Since $\mathcal{C}\left(e^{q_{1}}\right) \supseteq \mathcal{C}\left(e^{q_{2}}\right) \supseteq e^{q_{1}-q_{2}} \mathcal{C}\left(e^{q_{1}}\right)$ for each choice of $q_{2} \geq q_{1} \geq 0$, we have

$$
L_{j}\left(q_{1}\right) \leq L_{j}\left(q_{2}\right) \leq L_{j}\left(q_{1}\right)+\left(q_{2}-q_{1}\right) \quad\left(1 \leq j \leq N, 0 \leq q_{1} \leq q_{2}\right) .
$$

Thus $L_{1}, \ldots, L_{N}$ are continuous. The inequality $\log \|\mathbf{x}\| \leq \lambda_{\mathbf{x}}(q)$, valid for $q \geq 0$ and $\mathbf{x} \in V \backslash\{0\}$, shows that any bounded region of $[0, \infty) \times \mathbb{R}$ meets only finitely trajectories of points $\mathbf{x} \in \Lambda \backslash\{0\}$. As the latter cover the graphs of $L_{1}, \ldots, L_{N}$ and consist of at most two line segments of slope 0 and 1 , we conclude that $L_{1}, \ldots, L_{N}$ are piecewise linear with slopes 0 and 1. Finally, suppose that $L_{1}$ changes slope from 1 to 0 at a point $q>0$. Then, there exist $\epsilon>0$ and two non-zero points $\mathbf{x}$ and $\mathbf{y}$ in $\Lambda$ such that

$$
L_{1}(t)= \begin{cases}L_{\mathbf{x}}(t) & \text { for } t \in[q-\epsilon, q], \\ L_{\mathbf{y}}(t) & \text { for } t \in[q, q+\epsilon]\end{cases}
$$

This implies that $L_{\mathbf{x}}^{\prime}(q)=1$ and $L_{\mathbf{y}}^{\prime}(q)=0$. So, the points $\mathbf{x}$ and $\mathbf{y}$ are linearly independent. As they both belong to $\exp \left(L_{1}(q)\right) \mathcal{C}\left(e^{q}\right)$, we conclude that $L_{2}(q)=L_{1}(q)$.

2.3. The main family of convex bodies. Fix an integer $n \geq 2$ and a unit vector $\mathbf{u}$ of $\mathbb{R}^{n}$. We apply the preceding considerations to the decomposition

$$
\mathbb{R}^{n}=U \perp W \quad \text { where } \quad W=\langle\mathbf{u}\rangle_{\mathbb{R}} \quad \text { and } \quad U:=W^{\perp}=\left\{\mathbf{x} \in \mathbb{R}^{n} ; \mathbf{x} \cdot \mathbf{u}=0\right\},
$$

using the standard integer lattice $\Lambda=\mathbb{Z}^{n}$. Since $\left\|\operatorname{proj}_{W}(\mathbf{x})\right\|=|\mathbf{x} \cdot \mathbf{u}|$ for each $\mathbf{x} \in \mathbb{R}^{n}$, this gives rise to the family of convex bodies

$$
\mathcal{C}_{\mathbf{u}}(Q):=\left\{\mathbf{x} \in \mathbb{R}^{n} ;\|\mathbf{x}\| \leq 1 \text { and }|\mathbf{x} \cdot \mathbf{u}| \leq Q^{-1}\right\} \quad(Q \geq 1)
$$

and its associated map $\mathbf{L}_{\mathbf{u}}=\left(L_{\mathbf{u}, 1}, \ldots, L_{\mathbf{u}, n}\right):[0, \infty) \rightarrow \Delta_{n}$ where $L_{\mathbf{u}, j}(q)=\log \lambda_{j}\left(\mathcal{C}_{\mathbf{u}}\left(e^{q}\right)\right)$. By Lemmas 2.1 and 2.2, the functions $L_{\mathbf{u}, 1}, \ldots, L_{\mathbf{u}, n}$ are continuous, piecewise linear with slopes 0 and 1 , and they satisfy

$$
\left|L_{\mathbf{u}, 1}(q)+\cdots+L_{\mathbf{u}, n}(q)-q\right| \leq n \log (n) \quad(q \geq 0) .
$$

In particular, these functions are monotone increasing. By (2.1), the trajectory of a non-zero point $\mathbf{x} \in \mathbb{Z}^{n}$, with respect to the family of convex bodies $\mathcal{C}_{\mathbf{u}}$, is the graph of the function $L_{\mathbf{x}}:[0, \infty) \rightarrow \mathbb{R}$ given by

$$
L_{\mathbf{x}}(q)=L(\mathbf{x}, q):=\max \{\log \|\mathbf{x}\|, q+\log |\mathbf{x} \cdot \mathbf{u}|\} \quad(q \geq 0) .
$$

Equivalently, we note that $\lambda\left(\mathbf{x}, \mathcal{C}_{\mathbf{u}}(Q)\right)=\max \{\|\mathbf{x}\|, Q|\mathbf{x} \cdot \mathbf{u}|\} \quad(Q \geq 1)$. 
2.4. Families of pseudo-compound convex bodies. Let $\mathbf{u}$ and $U$ be as in $₫ 2.3$. We fix an integer $k \in\{1, \ldots, n\}$ and endow the vector space $V=\bigwedge^{k} \mathbb{R}^{n}$ with the unique structure of Euclidean space such that, for any orthonormal basis $\left(\mathbf{e}_{1}, \ldots, \mathbf{e}_{n}\right)$ of $\mathbb{R}^{n}$, the products $\mathbf{e}_{j_{1}} \wedge \cdots \wedge \mathbf{e}_{j_{k}}$ with $1 \leq j_{1}<\cdots<j_{k} \leq n$ form an orthonormal basis of $\bigwedge^{k} \mathbb{R}^{n}$. We also define $\Lambda=\Lambda^{k} \mathbb{Z}^{n}$ to be the lattice of co-volume 1 spanned by all products $\mathbf{x}_{1} \wedge \cdots \wedge \mathbf{x}_{k}$ with $\mathbf{x}_{1}, \ldots, \mathbf{x}_{k} \in \mathbb{Z}^{n}$. We have the orthogonal sum decomposition

$$
\bigwedge^{k} \mathbb{R}^{n}=U^{(k)} \perp W^{(k)} \quad \text { where } \quad U^{(k)}=\bigwedge^{k} U \text { and } \quad W^{(k)}=\bigwedge^{k-1} U \wedge\langle\mathbf{u}\rangle_{\mathbb{R}}
$$

In accordance with the general construction of $\$ 2.1$, we set

$$
N:=\operatorname{dim}_{\mathbb{R}}\left(\bigwedge^{k} \mathbb{R}^{n}\right)=\left(\begin{array}{l}
n \\
k
\end{array}\right) \quad \text { and } \quad K:=\operatorname{dim}_{\mathbb{R}}\left(W^{(k)}\right)=\left(\begin{array}{l}
n-1 \\
k-1
\end{array}\right)
$$

and, for each $Q \geq 1$, we define

$$
\mathcal{C}_{\mathbf{u}}^{(k)}(Q)=\left\{\omega \in \bigwedge^{k} \mathbb{R}^{n} ;\|\omega\| \leq 1 \text { and }\left\|\operatorname{proj}_{W^{(k)}}(\omega)\right\| \leq Q^{-1}\right\} .
$$

We also form the associated map $\mathbf{L}_{\mathbf{u}}^{(k)}=\left(L_{\mathbf{u}, 1}^{(k)}, \ldots, L_{\mathbf{u}, N}^{(k)}\right):[0, \infty) \rightarrow \Delta_{N}$ given by

$$
L_{\mathbf{u}, j}^{(k)}(q)=\log \lambda_{j}\left(\mathcal{C}_{\mathbf{u}}^{(k)}\left(e^{q}\right)\right) \quad(q \geq 0,1 \leq j \leq N) .
$$

By Lemmas 2.1 and 2.2, its components are continuous piecewise linear functions with slopes 0 and 1 , and they satisfy

$$
\left|L_{\mathbf{u}, 1}^{(k)}(q)+\cdots+L_{\mathbf{u}, N}^{(k)}(q)-K q\right| \leq N \log (N) \quad(q \geq 0) .
$$

Moreover we have the equality $L_{\mathbf{u}, 2}^{(k)}(q)=L_{\mathbf{u}, 1}^{(k)}(q)$ at each point $q>0$ where $L_{\mathbf{u}, 1}^{(k)}$ changes slope from 1 to 0 .

When $k=1$, we have $\bigwedge^{1} \mathbb{R}^{n}=\mathbb{R}^{n}, W^{(1)}=\langle\mathbf{u}\rangle_{\mathbb{R}}, \mathcal{C}_{\mathbf{u}}^{(1)}(Q)=\mathcal{C}_{\mathbf{u}}(Q)(Q \geq 1)$, and $\mathbf{L}_{\mathbf{u}}^{(1)}=\mathbf{L}_{\mathbf{u}}$. In general, we use $\mathcal{C}_{\mathbf{u}}^{(k)}(Q)$ as an approximation of the $k$-th compound convex body of $\mathcal{C}_{\mathbf{u}}(Q)$, namely the convex hull of the exterior products of $k$ elements of $\mathcal{C}_{\mathbf{u}}(Q)$ (see [1, Lemma 3]). The next lemma shows that this compound body is contained in $k \mathcal{C}_{\mathbf{u}}^{(k)}(Q)$.

Lemma 2.3. Let $Q \geq 1$ and let $\mathbf{x}_{1}, \ldots, \mathbf{x}_{k} \in \mathcal{C}_{\mathbf{u}}(Q)$. Then $\mathbf{x}_{1} \wedge \cdots \wedge \mathbf{x}_{k} \in k \mathcal{C}_{\mathbf{u}}^{(k)}(Q)$.

Proof. For $j=1, \ldots, k$, define $\mathbf{y}_{j}=\operatorname{proj}_{U}\left(\mathbf{x}_{j}\right)$ so that $\mathbf{x}_{j}=\mathbf{y}_{j}+\left(\mathbf{x}_{j} \cdot \mathbf{u}\right) \mathbf{u}$. Upon writing $\omega=\mathbf{x}_{1} \wedge \cdots \wedge \mathbf{x}_{k}$, we find

$$
\omega=\mathbf{y}_{1} \wedge \cdots \wedge \mathbf{y}_{k}+\sum_{j=1}^{k}(-1)^{j+k}\left(\mathbf{x}_{j} \cdot \mathbf{u}\right) \mathbf{y}_{1} \wedge \cdots \widehat{\mathbf{y}_{j}} \wedge \cdots \wedge \mathbf{y}_{k} \wedge \mathbf{u}
$$

Thus the sum on the right is $\operatorname{proj}_{W^{(k)}}(\omega)$ and therefore

$$
\left\|\operatorname{proj}_{W^{(k)}}(\omega)\right\| \leq \sum_{j=1}^{k}\left|\mathbf{x}_{j} \cdot \mathbf{u}\right|\left\|\mathbf{y}_{1}\right\| \cdots \widehat{\left\|\mathbf{y}_{j}\right\|} \cdots\left\|\mathbf{y}_{k}\right\| \leq k Q^{-1}
$$

As $\|\omega\| \leq\left\|\mathbf{x}_{1}\right\| \cdots\left\|\mathbf{x}_{k}\right\| \leq 1 \leq k$, we conclude that $\omega \in k \mathcal{C}_{\mathbf{u}}^{(k)}(Q)$. 
According to (2.1), the trajectory of a non-zero point $\omega \in \bigwedge^{k} \mathbb{Z}^{n}$ with respect to the family $\mathcal{C}_{\mathbf{u}}^{(k)}$ is the graph of the function $L_{\omega}:[0, \infty) \rightarrow \mathbb{R}$ given by

$$
L_{\omega}(q)=L(\omega, q):=\max \left\{\log \|\mathbf{x}\|, q+\log \left\|\operatorname{proj}_{W^{(k)}}(\omega)\right\|\right\} \quad(q \geq 0) .
$$

With this notation, the previous lemma generalizes as follows.

Lemma 2.4. Let $\mathbf{y}_{1}, \ldots, \mathbf{y}_{k}$ be linearly independent elements of $\mathbb{Z}^{n}$. We have

$$
L\left(\mathbf{y}_{1} \wedge \cdots \wedge \mathbf{y}_{k}, q\right) \leq L\left(\mathbf{y}_{1}, q\right)+\cdots+L\left(\mathbf{y}_{k}, q\right)+\log (k) \quad(q \geq 0) .
$$

Proof. Fix a choice of $q \geq 0$ and set $\omega=\mathbf{y}_{1} \wedge \cdots \wedge \mathbf{y}_{k}$. For each $j=1, \ldots, k$, we have $\lambda_{j}^{-1} \mathbf{y}_{j} \in$ $\mathcal{C}_{\mathbf{u}}\left(e^{q}\right)$ where $\lambda_{j}:=\exp \left(L\left(\mathbf{y}_{j}, q\right)\right)$. Thus the product $\left(\lambda_{1} \ldots \lambda_{k}\right)^{-1} \omega$ belongs to $k \mathcal{C}_{\mathbf{u}}^{(k)}\left(e^{q}\right)$, and so $L(\omega, q) \leq \log \left(k \lambda_{1} \ldots \lambda_{k}\right)$.

In the case where $k=n$, we have $U^{(n)}=0$. Then, our convex bodies are balls

$$
\mathcal{C}_{\mathbf{u}}^{(n)}(Q)=\left\{\omega \in \bigwedge^{n} \mathbb{R}^{n} ;\|\omega\| \leq Q^{-1}\right\} \quad(Q \geq 1)
$$

So we find $L(\omega, q)=q+\log \|\omega\|$ for any non-zero $\omega \in \bigwedge^{n} \mathbb{R}^{n}$ and any $q \geq 0$. In particular, this gives $L_{\mathbf{u}, 1}^{(n)}(q)=q(q \geq 0)$, and the preceding lemma admits the following consequence.

Lemma 2.5. Let $\mathbf{y}_{1}, \ldots, \mathbf{y}_{n}$ be linearly independent elements of $\mathbb{Z}^{n}$. We have

$$
\log \left\|\mathbf{y}_{1} \wedge \cdots \wedge \mathbf{y}_{n}\right\| \leq L\left(\mathbf{y}_{1}, q\right)+\cdots+L\left(\mathbf{y}_{n}, q\right)-q+\log (n) \quad(q \geq 0) .
$$

The next estimate essentially goes back to Mahler [9] (see also [3, §15.2]).

Lemma 2.6. For each $q \geq 0$, let $\left(S_{\mathbf{u}, 1}^{(k)}(q), \ldots, S_{\mathbf{u}, N}^{(k)}(q)\right)$ denote the sequence of all sums $L_{\mathbf{u}, j_{1}}(q)+\cdots+L_{\mathbf{u}, j_{k}}(q)$ with $1 \leq j_{1}<\cdots<j_{k} \leq n$ written in monotone increasing order. Then, we have

$$
-\log (n) \leq S_{\mathbf{u}, j}^{(k)}(q)-L_{\mathbf{u}, j}^{(k)}(q) \leq 2^{n} n \log (n) \quad(q \geq 0,1 \leq j \leq N) .
$$

Proof. Fix a choice of $q \geq 0$ and choose linearly independent points $\mathbf{y}_{1}, \ldots, \mathbf{y}_{n}$ of $\mathbb{Z}^{n}$ which realize the successive minima of $\mathcal{C}_{\mathbf{u}}\left(e^{q}\right)$ in the sense that $L\left(\mathbf{y}_{j}, q\right)=L_{\mathbf{u}, j}(q)$ for $j=1, \ldots, n$. Then, denote by $\left(\mu_{1}, \ldots, \mu_{N}\right)$ the set of numbers $L\left(\mathbf{y}_{j_{1}} \wedge \cdots \wedge \mathbf{y}_{j_{k}}, q\right)$ arranged in monotone increasing order (with the convention that $1 \leq j_{1}<\cdots<j_{k} \leq n$ ). Since the products $\mathbf{y}_{j_{1}} \wedge \cdots \wedge \mathbf{y}_{j_{k}}$ are linearly independent elements of $\wedge^{k} \mathbb{Z}^{n}$, we have $L_{\mathbf{u}, j}^{(k)}(q) \leq \mu_{j}$ for $j=$ $1, \ldots, N$. By Lemma 2.4, we also have

$$
L\left(\mathbf{y}_{j_{1}} \wedge \cdots \wedge \mathbf{y}_{j_{k}}, q\right) \leq L_{\mathbf{u}, j_{1}}(q)+\cdots+L_{\mathbf{u}, j_{k}}(q)+\log k \quad\left(1 \leq j_{1}<\cdots<j_{k} \leq n\right),
$$

and so $\mu_{j} \leq S_{\mathbf{u}, j}^{(k)}(q)+\log (k)$ for $j=1, \ldots, N$. Combining these two observations gives

$$
L_{\mathbf{u}, j}^{(k)}(q) \leq S_{\mathbf{u}, j}^{(k)}(q)+\log (k) \quad(1 \leq j \leq N) .
$$

On the other hand, the estimates (2.2) and (2.4) yield

$$
\sum_{j=1}^{N}\left(S_{\mathbf{u}, j}^{(k)}(q)+\log (k)-L_{\mathbf{u}, j}^{(k)}(q)\right)=K \sum_{j=1}^{n} L_{\mathbf{u}, j}(q)+N \log (k)-\sum_{j=1}^{N} L_{\mathbf{u}, j}^{(k)}(q) \leq c
$$

where $c=K n \log (n)+N \log (k)+N \log (N)=K n \log (n)+N \log (k N)$. Since $n K=k N \leq n^{k}$, we have $c \leq 2 K n \log (n) \leq 2^{n} n \log (n)$, and the conclusion follows. 
We simply need the following consequence of the previous lemma.

Lemma 2.7. Put $c_{1}=2^{n} n \log (n)$. For each $q \geq 0$, we have

(i) $\left|L_{\mathbf{u}, 1}^{(k)}(q)-L_{\mathbf{u}, 1}(q)-\cdots-L_{\mathbf{u}, k}(q)\right| \leq c_{1}$,

(ii) $\left|L_{\mathbf{u}, 2}^{(k)}(q)-L_{\mathbf{u}, 1}(q)-\cdots-L_{\mathbf{u}, k-1}(q)-L_{\mathbf{u}, k+1}(q)\right| \leq c_{1} \quad$ if $1<k<n$,

(iii) $\left|L_{\mathbf{u}, j}^{(n-1)}(q)+L_{\mathbf{u}, n+1-j}(q)-q\right| \leq c_{1}+n \log (n)$ for $j=1, \ldots, n$.

Proof. The first two estimates follow immediately from Lemma 2.6 because the smallest sum $L_{\mathbf{u}, j_{1}}(q)+\cdots+L_{\mathbf{u}, j_{k}}(q)$ is obtained by choosing $j_{1}=1, \ldots, j_{k}=k$, while the next one, when $1<k<n$, is obtained by choosing $j_{1}=1, \ldots, j_{k-1}=k-1$ and $j_{k}=k+1$. If $k=n-1$, then the $j$-th sum is $S_{\mathbf{u}, j}^{(n-1)}(q)=L_{\mathbf{u}, 1}(q)+\cdots+L_{\mathbf{u}, n}(q)-L_{\mathbf{u}, n+1-j}(q)$ and so (iii) follows from $(2.2)$.

2.5. The approximation theorem of Schmidt and Summerer. Fix an integer $n \geq 2$. The following definition is extracted from [14, §2] and adapted to our context.

Definition 2.8. Let $\gamma, q_{0} \geq 0$. An $(n, \gamma)$-system on the half-line $\left[q_{0}, \infty\right)$ is a function $\mathbf{P}=\left(P_{1}, \ldots, P_{n}\right):\left[q_{0}, \infty\right) \rightarrow \mathbb{R}^{n}$ which satisfies the following conditions.

$(\mathrm{S} 1)-\gamma \leq P_{j}(q) \leq P_{j+1}(q)+\gamma \quad\left(1 \leq j<n, q_{0} \leq q\right)$.

(S2) $P_{j}\left(q_{1}\right) \leq P_{j}\left(q_{2}\right)+\gamma \quad\left(1 \leq j \leq n, q_{0} \leq q_{1} \leq q_{2}\right)$.

(S3) For $j=1, \ldots, n$, the function $M_{j}:=P_{1}+\cdots+P_{j}:\left[q_{0}, \infty\right) \rightarrow \mathbb{R}$ is continuous and piecewise linear with slopes 0 and 1.

(S4) $M_{n}(q)=q \quad\left(q_{0} \leq q\right)$.

(S5) If, for $j \in\{1, \ldots, n-1\}$, the function $M_{j}$ changes slope from 1 to 0 at a point $q>q_{0}$, then $P_{j+1}(q) \leq P_{j}(q)+\gamma$.

Up to the value of $\gamma$, the next result comes from [14, §2]. It shows the fundamental importance of the notion of $(n, \gamma)$-systems. For the sake of completeness and because our context is slightly different, we also recall its proof below.

Theorem 2.9 (Schmidt-Summerer, 2013). Let $\gamma=6 n 2^{n} \log (n)$. For each unit vector $\mathbf{u}$ of $\mathbb{R}^{n}$, there exists an $(n, \gamma)$-system $\mathbf{P}:[0, \infty) \rightarrow \mathbb{R}^{n}$ such that

$$
\sup _{q \geq 0}\left\|\mathbf{P}(q)-\mathbf{L}_{\mathbf{u}}(q)\right\|_{\infty} \leq \gamma .
$$

Proof. Set $M_{0}:=0$ and $M_{k}:=L_{\mathbf{u}, 1}^{(k)}$ for $k=1, \ldots, n$. By Lemma 2.7 (i), we have

$$
\left|M_{k}(q)-L_{\mathbf{u}, 1}(q)-\cdots-L_{\mathbf{u}, k}(q)\right| \leq c_{1} \quad(1 \leq k \leq n, 0 \leq q) .
$$

Then, upon defining $P_{k}=M_{k}-M_{k-1}$ for $k=1, \ldots, n$, we obtain

$$
\left|P_{k}(q)-L_{\mathbf{u}, k}(q)\right| \leq 2 c_{1} \quad(1 \leq k \leq n, 0 \leq q)
$$


So, in order to complete the proof of the theorem, it suffices to show that $\mathbf{P}:=\left(P_{1}, \ldots, P_{n}\right)$ is an $\left(n, 6 c_{1}\right)$-system on $[0, \infty)$. The conditions (S1) and (S2) derive immediately from (2.6) because for each $q \geq 0$ we have

$$
\begin{aligned}
& P_{k}(q) \geq L_{\mathbf{u}, k}(q)-2 c_{1} \geq-2 c_{1}, \\
& P_{k}(q) \leq L_{\mathbf{u}, k}(q)+2 c_{1} \leq L_{\mathbf{u}, k+1}(q)+2 c_{1} \leq P_{k+1}(q)+4 c_{1} \quad(1 \leq k<n),
\end{aligned}
$$

while for $0 \leq q_{1} \leq q_{2}$ we find

$$
P_{k}\left(q_{1}\right) \leq L_{\mathbf{u}, k}\left(q_{1}\right)+2 c_{1} \leq L_{\mathbf{u}, k}\left(q_{2}\right)+2 c_{1} \leq P_{k}\left(q_{2}\right)+4 c_{1} \quad(1 \leq k \leq n) .
$$

For $k=1, \ldots, n$, we also have $P_{1}+\cdots+P_{k}=M_{k}-M_{0}=M_{k}$, and we know that $M_{k}=L_{\mathbf{u}, 1}^{(k)}$ is continuous and piecewise linear with slopes 0 and 1 . Moreover, as noticed in 92.4 , we have $M_{n}(q)=L_{\mathbf{u}, 1}^{(n)}(q)=q$ for each $q \geq 0$. Thus (S3) and (S4) are automatically satisfied. Finally, (S5) also holds because if, for some $k \in\{1, \ldots, n-1\}$, the function $M_{k}=L_{\mathbf{u}, 1}^{(k)}$ changes slope from 1 to 0 at a point $q>0$, then $L_{\mathbf{u}, 2}^{(k)}(q)=L_{\mathbf{u}, 1}^{(k)}(q)$ and so the estimates (i) and (ii) of Lemma 2.7 yield $L_{\mathbf{u}, k+1}(q)-L_{\mathbf{u}, k}(q) \leq 2 c_{1}$, which in turn implies that $P_{k+1}(q) \leq P_{k}(q)+6 c_{1}$.

Note that the properties of an $(n, \gamma)$-system are simpler when $\gamma=0$. For example, the condition (S3) implies that the components of an $(n, \gamma)$-system are continuous piecewise linear functions with slopes $-1,0$ or 1 . However, when $\gamma=0$, it follows from (S2) that these components are monotone increasing and so, possess only the slopes 0 and 1 . Moreover, (S1) shows that an $(n, 0)$-system takes values in $\Delta_{n}$ while this may also fail for a general $(n, \gamma)$-system. A general description of $(n, 0)$-systems is given in [14, §3]. We conclude this section with the following observation which describes the rigid $n$-systems as a subset of the set of $(n, 0)$-systems, leaving its proof to the reader.

Lemma 2.10. Let $\delta \in(0, \infty)$. The rigid $n$-systems of mesh $\delta$ are the $(n, 0)$-systems $\left(P_{1}, \ldots, P_{n}\right):\left[q_{0}, \infty\right) \rightarrow \mathbb{R}^{n}$ with the property that, for $q=q_{0}$ and for each $q>q_{0}$ at which at least one of the functions $P_{1}+\cdots+P_{j}(1 \leq j<n)$ changes slope from 0 to 1 , the numbers $P_{1}(q), \ldots, P_{n}(q)$ are $n$ distinct multiples of $\delta$.

In particular, a rigid system $\left(P_{1}, \ldots, P_{n}\right):\left[q_{0}, \infty\right) \rightarrow \mathbb{R}^{n}$ satisfies

$$
P_{1}(q)+\cdots+P_{n}(q)=q \quad\left(q \geq q_{0}\right) .
$$

This is an important property that we will use repeatedly.

\section{A sPeCial CASE}

In this section, we fix an integer $n \geq 2$ and assume that a rigid $n$-system $\mathbf{P}$ of large mesh is, in comparison, very closely approximated by the map $\mathbf{L}_{\mathbf{u}}$ for some unit vector $\mathbf{u}$ of $\mathbb{R}^{n}$. We derive from this the existence of a sequence of $n$-tuples of integer points with strong properties. This partly explains and motivates the constructions of Section 5 where, given an arbitrary rigid system $\mathbf{P}$ of sufficiently large mesh we construct a unit vector $\mathbf{u}$ such that $\mathbf{P}-\mathbf{L}_{\mathbf{u}}$ is bounded. Our goal is thus to prove the following complement to Theorem 1.3 . 
Theorem 3.1. Let $\delta, \epsilon$ be positive real numbers with $\delta \geq 6\left(n \epsilon+c_{1}\right)$ where $c_{1}$ is as in Lemma 2.7. and let $\mathbf{P}=\left(P_{1}, \ldots, P_{n}\right):\left[q_{0}, \infty\right) \rightarrow \mathbb{R}^{n}$ be a rigid system of mesh $\delta$. Suppose that there exists a unit vector $\mathbf{u} \in \mathbb{R}^{n}$ such that $\left\|\mathbf{P}(q)-\mathbf{L}_{\mathbf{u}}(q)\right\|_{\infty} \leq \epsilon$ for any $q \geq q_{0}$. Consider the sequences $\left(q_{i}\right)_{0 \leq i<s},\left(k_{i}\right)_{0 \leq i<s}$ and $\left(\ell_{i}\right)_{0 \leq i<s}$ attached to $\mathbf{P}$ as in Definition 1.2. and set $q_{s}=\infty$ if $s<\infty$. Then, for each integer $i$ with $0 \leq i<s$, there exists an $n$-tuple of linearly independent integer points $\left(\mathbf{x}_{1}^{(i)}, \ldots, \mathbf{x}_{n}^{(i)}\right)$ with the following properties:

1) $\left\|\mathbf{P}(q)-\Phi_{n}\left(L\left(\mathbf{x}_{1}^{(i)}, q\right), \ldots, L\left(\mathbf{x}_{n}^{(i)}, q\right)\right)\right\|_{\infty} \leq \epsilon \quad\left(q_{i} \leq q<q_{i+1}\right)$,

2) $\left(\mathbf{x}_{1}^{(i)}, \ldots, \widehat{\mathbf{x}_{k_{i}}^{(i)}}, \ldots, \mathbf{x}_{n}^{(i)}\right)=\left(\mathbf{x}_{1}^{(i+1)}, \ldots, \widehat{\mathbf{x}_{\ell_{i+1}}^{(i+1)}}, \ldots, \mathbf{x}_{n}^{(i+1)}\right)$ if $i+1<s$,

3) $\left|\log \left\|\mathbf{x}_{j}^{(i)}\right\|-P_{j}\left(q_{i}\right)\right| \leq \epsilon \quad(j=1, \ldots, n) \quad$ if $i \geq 1$,

4) $\mathbf{x}_{\ell_{i+1}}^{(i+1)} \in\left\langle\mathbf{x}_{1}^{(i)}, \ldots, \mathbf{x}_{\ell_{i+1}}^{(i)}\right\rangle_{\mathbb{R}}$ if $i+1<s$,

5) $0 \leq \log \left|\operatorname{det}\left(\mathbf{x}_{1}^{(i)}, \ldots, \mathbf{x}_{n}^{(i)}\right)\right| \leq n \epsilon+\log (n)$,

6) $\left|\log \left\|\mathbf{x}_{1}^{(i)} \wedge \cdots \wedge \widehat{\mathbf{x}_{k_{i}}^{(i)}} \wedge \cdots \wedge \mathbf{x}_{n}^{(i)}\right\|-\sum_{j \neq k_{i}} \log \left\|\mathbf{x}_{j}^{(i)}\right\|\right| \leq n \epsilon+2 c_{1}$ if $i \geq 1$.

The property 1) means that, over each interval $\left[q_{i}, q_{i+1}\right)$, the combined graph of $\mathbf{P}$ is contained in an $\epsilon$-neighborhood of the union of the trajectories of $\mathbf{x}_{1}^{(i)}, \ldots, \mathbf{x}_{n}^{(i)}$. Together with the hypothesis, it implies that, for each $q$ in that interval, the latter points realize the logarithms of the successive minima of $\mathcal{C}_{\mathbf{u}}\left(e^{q}\right)$ within $2 \epsilon$. Because of the specific form of the combined graph of $\mathbf{P}$, we also infer from 1) that exactly one of the points $\mathbf{x}_{j}^{(i)}$ must have a trajectory of slope 1 over $\left[q_{i}+2 \epsilon, \infty\right)$ while all other points have trajectories of slope 0 over $\left[0, q_{i+1}-2 \epsilon\right.$ ) (see Lemma 3.3). If $i+1<s$, it follows from 2) that this exceptional point must be $\mathbf{x}_{k_{i}}^{(i)}$. Over the next interval $\left[q_{i+1}, q_{i+2}\right)$, its trajectory is replaced by that of a new point $\mathbf{x}_{\ell_{i+1}}^{(i+1)}$ while those of the other points are kept. Figure 3 illustrates this on an example with $n=5$. In this picture the solid lines represent the combined graph of $\mathbf{P}$ and the dotted lines the trajectories of the points $\mathbf{x}_{j}^{(i)}$.

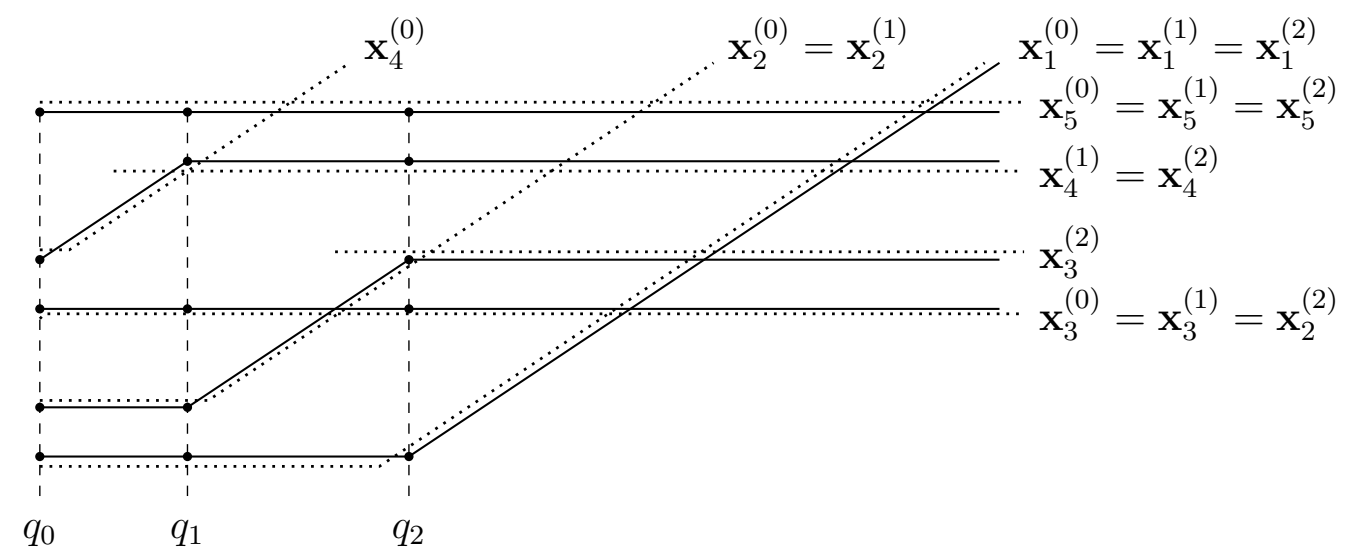

Figure 2. The combined graph of a rigid 5-system and the trajectories of integer points that approximate it within $\epsilon$. 
The properties 3) to 6) are useful additional information about the points $\mathbf{x}_{j}^{(i)}$. For example, 5) tells us that each $n$-tuple $\left(\mathbf{x}_{1}^{(i)}, \ldots, \mathbf{x}_{n}^{(i)}\right)$ spans a subgroup of $\mathbb{Z}^{n}$ with uniformly bounded index, while 6) implies that the angles between any two of the points $\mathbf{x}_{1}^{(i)}, \ldots, \widehat{\mathbf{x}_{k_{i}}^{(i)}}, \ldots, \mathbf{x}_{n}^{(i)}$ are bounded away from 0. In Section 5 , dealing with the inverse problem, we construct $n$-tuples of integer points with stronger properties. In particular, we request that each $n$-tuple $\left(\mathbf{x}_{1}^{(i)}, \ldots, \mathbf{x}_{n}^{(i)}\right)$ is a basis of $\mathbb{Z}^{n}$ and that the points $\mathbf{x}_{1}^{(i)}, \ldots, \widehat{\mathbf{x}_{k_{i}}^{(i)}}, \ldots, \mathbf{x}_{n}^{(i)}$ are almost orthogonal in a sense that is defined in $\$ 4$.

Preliminary observations towards the proof of Theorem 3.1 . Suppose that the hypotheses of Theorem 3.1 are fulfilled. For each $\mathbf{x} \in \mathbb{R}^{n} \backslash\{0\}$ and each $j \in\{1, \ldots, n\}$, we set

$$
L(\mathbf{x}, \infty)=\lim _{q \rightarrow \infty} L(\mathbf{x}, q), \quad L_{\mathbf{u}, j}(\infty)=\lim _{q \rightarrow \infty} L_{\mathbf{u}, j}(q), \quad P_{j}(\infty)=\lim _{q \rightarrow \infty} P_{j}(q) .
$$

We also define

$$
\begin{aligned}
& V_{j}(q)=\left\langle\mathbf{x} \in \mathbb{Z}^{n} \backslash\{0\} ; L(\mathbf{x}, q) \leq L_{\mathbf{u}, j}(q)\right\rangle_{\mathbb{R}} \quad(1 \leq j \leq n, 0 \leq q \leq \infty), \\
& V_{0}(q)=0 \quad(0 \leq q \leq \infty) .
\end{aligned}
$$

The vector space $V_{j}(\infty)$ is interesting only when $L_{\mathbf{u}, j}(\infty)<\infty$ or equivalently when $P_{j}(\infty)<$ $\infty$. In that case, $V_{j}(\infty)$ is orthogonal to $\mathbf{u}$ and we have $L(\mathbf{x}, q)=\log \|\mathbf{x}\| \quad(q \geq 0)$ for any $\mathbf{x} \in V_{j}(\infty) \backslash\{0\}$. Otherwise, we have $V_{j}(\infty)=\mathbb{R}^{n}$. We start with three observations.

Lemma 3.2. Let $j \in\{1, \ldots, n-1\}$. The family of vector spaces $V_{j}(q)$ is constant, with dimension $j$, on any subinterval of $\left[q_{0}, \infty\right)$ on which $P_{j+1}(q)>P_{j}(q)+2 \epsilon$.

Proof. Since $\left\|\mathbf{P}(q)-\mathbf{L}_{\mathbf{u}}(q)\right\|_{\infty} \leq \epsilon$ for each $q \geq q_{0}$, the condition $P_{j+1}(q)>P_{j}(q)+2 \epsilon$ implies that $L_{\mathbf{u}, j+1}(q)>L_{\mathbf{u}, j}(q)$ and thus $\operatorname{dim}_{\mathbb{R}} V_{j}(q)=j$. For any given $q \geq q_{0}$ with this property, there exists a connected neighborhood $I$ of $q$ in $\left[q_{0}, \infty\right)$ and a real number $r$ such that $L_{\mathbf{u}, j}(t)<r<L_{\mathbf{u}, j+1}(t)$ for each $t \in I$. Then we have

$$
V_{j}(t)=\left\langle\mathbf{x} \in \mathbb{Z}^{n} \backslash\{0\} ; L(\mathbf{x}, t)<r\right\rangle_{\mathbb{R}} \quad(t \in I)
$$

and so the family $V_{j}(t)$ is monotone decreasing on $I$. As it has constant dimension $j$, it is therefore constant. Thus the family $V_{j}(q)$ is locally constant and therefore constant on any subinterval of $\left[q_{0}, \infty\right)$ on which $P_{j+1}>P_{j}+2 \epsilon$.

Lemma 3.3. Let $i$ be an integer with $0 \leq i<s$. Put $k=k_{i}$. Then, there exists a point $\mathbf{x} \in \mathbb{Z}^{n}$ with $\mathbf{x} \notin V_{k-1}\left(q_{i}\right)$ such that

$$
\left|L(\mathbf{x}, t)-\left(P_{k}\left(q_{i}\right)+\max \left\{0, t-q_{i}\right\}\right)\right| \leq \epsilon \quad(t \geq 0) .
$$

In particular, the trajectory of $\mathbf{x}$ has slope 0 on $\left[0, q_{i}-2 \epsilon\right]$ if $i \geq 1$ and slope 1 on $\left[q_{i}+2 \epsilon, \infty\right)$.

Proof. Let $I=\left\{t \geq q_{0} ;\left|t-q_{i}\right|<\delta-2 \epsilon\right\}$. If $k \geq 2$, we have $P_{k}\left(q_{i}\right) \geq P_{k-1}\left(q_{i}\right)+\delta$ and so $P_{k}(t)>P_{k-1}(t)+2 \epsilon$ for any $t \in I$. By Lemma 3.2, this implies that $V_{k-1}(t)=V_{k-1}\left(q_{i}\right)$ has dimension $k-1$ for each $t \in I$. This is also true if $k=1$ because then $V_{k-1}(t)=0$ for each $t \geq 0$. 
Since $\operatorname{dim}_{\mathbb{R}} V_{k-1}\left(q_{i}\right)=k-1$, there exists $\mathbf{x} \in \mathbb{Z}^{n}$ with $\mathbf{x} \notin V_{k-1}\left(q_{i}\right)$ such that $L\left(\mathbf{x}, q_{i}\right)=$ $L_{\mathbf{u}, k}\left(q_{i}\right)$. We claim that this point satisfies the condition (3.1). For each $t \in I$, we have $\mathbf{x} \notin V_{k-1}(t)$, and therefore

$$
L(\mathbf{x}, t) \geq L_{\mathbf{u}, k}(t) \geq P_{k}(t)-\epsilon \quad(t \in I) .
$$

As $L(\mathbf{x}, t)$ has slope 0 and 1 , we also have

$$
L(\mathbf{x}, t) \leq L\left(\mathbf{x}, q_{i}\right)+\max \left\{0, t-q_{i}\right\} \quad(t \geq 0) .
$$

By construction, the function $P_{k}$ is constant on $\left[q_{i}-\delta, q_{i}\right]$ if $i \geq 1$, and has slope 1 on $\left[q_{i}, q_{i}+\delta\right]$. Since $L\left(\mathbf{x}, q_{i}\right)=L_{\mathbf{u}, k}\left(q_{i}\right) \leq P_{k}\left(q_{i}\right)+\epsilon$, the previous inequality yields

$$
L(\mathbf{x}, t) \leq P_{k}\left(q_{i}\right)+\max \left\{0, t-q_{i}\right\}+\epsilon=P_{k}(t)+\epsilon \quad(t \in I) .
$$

Thus, (3.1) holds for each $t \in I$. Since $\delta>4 \epsilon$, this implies that $L(\mathbf{x}, t)$ is constant on $\left[0, q_{i}-2 \epsilon\right]$ if $i \geq 1$, and that it has slope 1 on $\left[q_{i}+2 \epsilon, \infty\right)$. Thus the validity of (3.1) extends from $I$ to the whole interval $[0, \infty)$.

Lemma 3.4. Suppose that $P_{j}(\infty)<\infty$ for some index $j$ with $1 \leq j<n$. Then $V_{j}(\infty)$ has dimension $j$ and there exists a real number $q \geq 0$ such that $V_{j}(t)=V_{j}(\infty)$ for each $t \geq q$. Moreover, there exists a point $\mathbf{x} \in \mathbb{Z}^{n} \cap V_{j}(\infty)$ with $\mathbf{x} \notin V_{j-1}(\infty)$ such that $\left|L(\mathbf{x}, t)-P_{j}(\infty)\right| \leq$ $\epsilon$ for each $t \geq 0$.

Proof. Given a real number $q \geq 0$, there are finitely many non-zero points $\mathbf{x} \in \mathbb{Z}^{n}$ such that $L(\mathbf{x}, t) \leq L_{\mathbf{u}, j}(t)$ for some $t \geq q$ because this implies that $\log \|\mathbf{x}\| \leq P_{j}(t)+\epsilon \leq P_{j}(\infty)+\epsilon$. Moreover, the set of these points $\mathbf{x}$ is monotone decreasing as a function of $q$. Choose $q$ so that it has minimal cardinality. Then, all points $\mathbf{x}$ with the above property have $L(\mathbf{x}, t)$ constant, equal to $\log \|\mathbf{x}\|$, for $t \geq q$. Thus, $L_{\mathbf{u}, j}$ is also constant on $[q, \infty)$ and we find

$$
V_{j}(t)=\left\langle\mathbf{x} \in \mathbb{Z}^{n} \backslash\{0\} ; \mathbf{x} \cdot \mathbf{u}=0, \log \|\mathbf{x}\| \leq L_{\mathbf{u}, j}(\infty)\right\rangle_{\mathbb{R}}=V_{j}(\infty)
$$

for each $t \geq q$. If $q$ is sufficiently large, we also have $P_{j+1}(q)>P_{j}(q)+2 \epsilon$ because $P_{j+1}(\infty)>$ $P_{j}(\infty)+\delta$. Then, $V_{j}(\infty)=V_{j}(q)$ has dimension $j$ according to Lemma 3.2 .

By the above, we also have $\operatorname{dim}_{\mathbb{R}} V_{j-1}(\infty)=j-1$ if $j \geq 2$, because then $P_{j-1}(\infty)<\infty$. This remains true if $j=1$ since $V_{0}(\infty)=0$. Thus $V_{j}(\infty) \neq V_{j-1}(\infty)$. Then, assuming $q$ sufficiently large so that $V_{j-1}(q)=V_{j-1}(\infty)$, we have $V_{j}(q) \neq V_{j-1}(q)$ and consequently there exists $\mathbf{x} \in \mathbb{Z}^{n} \cap V_{j}(q)$ with $\mathbf{x} \notin V_{j-1}(q)$ such that $L(\mathbf{x}, q)=L_{\mathbf{u}, j}(q)$. This point has the requested property because $L(\mathbf{x}, t)=L_{\mathbf{u}, j}(\infty)$ for $t \geq 0$, and $\left|L_{\mathbf{u}, j}(\infty)-P_{j}(\infty)\right| \leq \epsilon$.

Thanks to Lemmas 3.2 and 3.4 , we note that $\operatorname{dim}_{\mathbb{R}} V_{j}\left(q_{i}\right)=j$ for each pair of integers $i$ $(\neq \infty)$ and $j$ with $0 \leq i \leq s$ and $0 \leq j \leq n$.

Construction of the points $\mathrm{x}_{j}^{(i)}$ and proof of Properties 1-3). We denote by $\mathcal{S}$ the set of all maximal horizontal line segments of positive length contained in the combined graph of $\mathbf{P}$, together with the point $\left(q_{0}, P_{k_{0}}\left(q_{0}\right)\right)$ (which is thus the only line segment of length 0 in $\mathcal{S}$.

To each $S \in \mathcal{S}$ we associate a point $\mathbf{x}_{S} \in \mathbb{Z}^{n}$ in the following way. If $S$ is bounded, its right end-point is $\left(q_{i}, P_{k_{i}}\left(q_{i}\right)\right)$ for some integer $i$ with $0 \leq i<s$. Then, we choose for $\mathbf{x}_{S}$ any point $\mathbf{x}$ with the property stated in Lemma [3.3. Otherwise, $S$ is contained in $[0, \infty) \times\left\{P_{j}(\infty)\right\}$ 
for some index $j$ with $1 \leq j<n$ such that $P_{j}(\infty)<\infty$. Then, we choose for $\mathbf{x}_{S}$ any point $\mathbf{x}$ with the property stated in Lemma 3.4.

Finally, for each pair of integers $i$ and $j$ with $0 \leq i<s$ and $1 \leq j \leq n$, there exists a unique line segment $S$ in $\mathcal{S}$ containing the point $\left(q_{i}, P_{j}\left(q_{i}\right)\right)$ and we define $\mathbf{x}_{j}^{(i)}=\mathbf{x}_{S}$. In the degenerate case where $s<\infty$, we have $P_{j}(\infty)<\infty$ for each $j=1, \ldots, n-1$. Then, for those $j$, we define $\mathbf{x}_{j}^{(s)}=\mathbf{x}_{S}$ where $S$ is the unique unbounded line segment of $\mathcal{S}$ contained in $[0, \infty) \times\left\{P_{j}(\infty)\right\}$. By construction, these points $\mathbf{x}_{1}^{(s)}, \ldots, \mathbf{x}_{n-1}^{(s)}$ are linearly independent.

With these definitions, Property 2) in Theorem 3.1 is automatically satisfied. More precisely we have

$$
\left(\mathbf{x}_{1}^{(i)}, \ldots, \widehat{\mathbf{x}_{k_{i}}^{(i)}}, \ldots, \mathbf{x}_{n}^{(i)}\right)=\left(\mathbf{x}_{1}^{(i+1)}, \ldots, \widehat{\mathbf{x}_{\ell_{i+1}}^{(i+1)}}, \ldots, \mathbf{x}_{n}^{(i+1)}\right) \quad(0 \leq i<s)
$$

upon defining $\ell_{s}=n$ if $s<\infty$. Property 1) is also clear in view of the description of the trajectories of the points $\mathbf{x}$ given by Lemmas 3.3 and 3.4, as illustrated on Figure 3 for $n=5$. Indeed, for any fixed index $i$ with $0 \leq i<s$, we have

$$
\begin{aligned}
& \sup _{q_{i} \leq t<q_{i+1}}\left|L\left(\mathbf{x}_{k_{i}}^{(i)}, t\right)-P_{k_{i}}\left(q_{i}\right)-\left(t-q_{i}\right)\right| \leq \epsilon, \\
& \sup _{q_{i} \leq t<q_{i+1}}\left|L\left(\mathbf{x}_{j}^{(i)}, t\right)-P_{j}\left(q_{i}\right)\right| \leq \epsilon \quad\left(1 \leq j \leq n, j \neq k_{i}\right) .
\end{aligned}
$$

Since $\mathbf{P}(t)=\Phi_{n}\left(P_{1}\left(q_{i}\right), \ldots, \widehat{P_{k_{i}}\left(q_{i}\right)}, \ldots, P_{n}\left(q_{i}\right), P_{k_{i}}\left(q_{i}\right)+t-q_{i}\right)$ for each $t \in\left[q_{i}, q_{i+1}\right)$, and since $\left\|\Phi(\mathbf{p})-\Phi\left(\mathbf{p}^{\prime}\right)\right\|_{\infty} \leq\left\|\mathbf{p}-\mathbf{p}^{\prime}\right\|_{\infty}$ for any $\mathbf{p}, \mathbf{p}^{\prime} \in \mathbb{R}^{n}$, this yields 1$)$. Moreover, if $i \geq 1$, we have $\left|L\left(\mathbf{x}_{j}^{(i)}, t\right)-P_{j}\left(q_{i}\right)\right| \leq \epsilon$ for each $t \in\left[0, q_{i}\right]$ and each $j=1, \ldots, n$. Upon setting $=0$, this yields Property 3$)$.

Linear independence of the $n$-tuples $\left(\mathbf{x}_{1}^{(i)}, \ldots, \mathbf{x}_{n}^{(i)}\right)$. This is the most delicate part of the argument. It will come out of the last of the following four lemmas.

Lemma 3.5. Let $i, j$ be integers with $0 \leq i<s$ and $0 \leq j<n$. If $P_{j+1}$ is constant on $\left[q_{i}, \infty\right]$, then $V_{j}\left(q_{i}\right)=V_{j}(\infty)$. If $P_{j+1}$ is constant on $\left[q_{i}, q_{r}\right]$ for some integer $r$ with $i \leq r<s$, then $V_{j}\left(q_{i}\right)=V_{j}\left(q_{r}\right)$.

Proof. If $j=0$, this is clear since $V_{0}(q)=0$ for any $q \in[0, \infty]$. Suppose that $j \geq 1$. In the first case, we choose $q \geq q_{i}$ sufficiently large so that, by Lemma 3.4, we have $V_{j}(q)=V_{j}(\infty)$. In the second case, we set $q=q_{r}$. In both cases, we have $P_{j+1}(t) \geq P_{j}(t)+\delta$ for each $t \in\left[q_{i}, q\right]$ and so, by Lemma [3.2, we conclude that $V_{j}\left(q_{i}\right)=V_{j}(q)$.

Lemma 3.6. Let $i, j$ be integers with $0 \leq i \leq s$ and $0 \leq j \leq n$, and let $\omega$ be a generator of the one-dimensional vector space $\bigwedge^{j} V_{j}\left(q_{i}\right)$.

1) If $0<i \leq s$ and $j<\ell_{i}$, then $L(\omega, t)$ is constant on $\left[0, q_{i}-\delta / 3\right]$ (understood as $[0, \infty]$ if $i=s$, since in that case $\left.q_{s}=\infty\right)$.

2) If $0 \leq i<s$ and $j \geq k_{i}$, then $L(\omega, t)$ has slope 1 on $\left[q_{i}+\delta / 3, \infty\right)$.

Proof. Since $V_{j}\left(q_{i}\right)$ has dimension $j$, its $j$-th exterior power has dimension 1 . For any other generator $\omega^{\prime}$ of the latter vector space, the difference $L(\omega, t)-L\left(\omega^{\prime}, t\right)$ is constant. So, we 
may assume that $\omega=\mathbf{y}_{1} \wedge \cdots \wedge \mathbf{y}_{j}$ where $\mathbf{y}_{1}, \ldots, \mathbf{y}_{j}$ are linearly independent points of $\mathbb{Z}^{n}$ such that $L\left(\mathbf{y}_{m}, q_{i}\right)=L_{\mathbf{u}, m}\left(q_{i}\right)$ for $m=1, \ldots, j$.

If $i=s$ and $j<\ell_{s}=n$, then $\mathbf{y}_{1}, \ldots, \mathbf{y}_{j}$ belong to $U=\mathbf{u}^{\perp}$ (because $V_{j}(\infty) \subseteq U$ ) and so $\omega \in \bigwedge^{j} U$. By virtue of formula (2.5) with $k$ replaced by $j$, this implies that $L(\omega, t)=\log \|\omega\|$ is constant for all $t \geq 0$.

From now on, we may therefore assume that $i<s$, and so $q_{i}<\infty$. As $\omega$ is a non-zero element of $\bigwedge^{j} \mathbb{Z}^{n}$ we have

$$
L(\omega, t) \geq L_{\mathbf{u}, 1}^{(j)}(t) \quad(t \geq 0) .
$$

By Lemma 2.7 (i), we also have

$$
L_{\mathbf{u}, 1}^{(j)}(t) \geq L_{\mathbf{u}, 1}(t)+\cdots+L_{\mathbf{u}, j}(t)-c_{1} \geq M_{j}(t)-j \epsilon-c_{1} \quad\left(t \geq q_{0}\right),
$$

where $M_{j}=P_{1}+\cdots+P_{j}$. On the other hand, since $L(\omega, t)$ has slopes 0 and 1 , it satisfies

$$
L(\omega, t) \leq L\left(\omega, q_{i}\right)+\max \left\{0, t-q_{i}\right\} \quad(t \geq 0),
$$

while Lemma 2.4 gives

$$
\begin{aligned}
L\left(\omega, q_{i}\right) \leq & L\left(\mathbf{y}_{1}, q_{i}\right)+\cdots+L\left(\mathbf{y}_{j}, q_{i}\right)+\log n \\
& =L_{\mathbf{u}, 1}\left(q_{i}\right)+\cdots+L_{\mathbf{u}, j}\left(q_{i}\right)+\log n \leq M_{j}\left(q_{i}\right)+j \epsilon+c_{1} .
\end{aligned}
$$

Combining these estimates, we conclude that

$$
\left|L(\omega, t)-M_{j}(t)\right| \leq\left|M_{j}\left(q_{i}\right)+\max \left\{0, t-q_{i}\right\}-M_{j}(t)\right|+(\delta / 6) \quad\left(t \geq q_{0}\right)
$$

since $j \epsilon+c_{1} \leq \delta / 6$. If $0<i<s$ and $j<\ell_{i}$, then $M_{j}$ is constant on $\left[q_{i}-\delta\right.$, $\left.q_{i}\right]$ and the latter estimate gives $\left|L(\omega, t)-M_{j}\left(q_{i}\right)\right| \leq \delta / 6$ for each $t \in\left[q_{i}-\delta, q_{i}\right]$. So, $L(\omega, t)$ is constant on $\left[0, q_{i}-\delta / 3\right]$. If $0 \leq i<s$ and $j \geq k_{i}$, then $M_{j}$ has slope 1 on $\left[q_{i}, q_{i}+\delta\right]$ and the same estimate yields $\left|L(\omega, t)-M_{j}(t)\right| \leq \delta / 6$ for each $t \in\left[q_{i}, q_{i}+\delta\right]$. Then, $L(\omega, t)$ has slope 1 on $\left[q_{i}+\delta / 3, \infty\right)$.

Lemma 3.7. Let $i, j \in \mathbb{N}$ with $0 \leq i<s$ and $k_{i} \leq j<\ell_{i+1}$. Then, $V_{j}\left(q_{i}\right) \neq V_{j}\left(q_{i+1}\right)$.

Proof. Suppose on the contrary that $V_{j}\left(q_{i}\right)=V_{j}\left(q_{i+1}\right)$ and let $\omega$ be a generator of the $j$-th exterior power of this vector space. By Lemma 3.6 applied successively to the points $q_{i}$ and $q_{i+1}$, the function $L(\omega, t)$ has slope 1 on $\left[q_{i}+\delta / 3, \infty\right)$ and is constant on $\left[0, q_{i+1}-\delta / 3\right]$. As $q_{i+1} \geq q_{i}+\delta$, this is impossible.

Lemma 3.8. For each pair of integers $i, j$ with $0 \leq i<s$ and $0 \leq j \leq n$, we have $V_{j}\left(q_{i}\right)=\left\langle\mathbf{x}_{1}^{(i)}, \ldots, \mathbf{x}_{j}^{(i)}\right\rangle_{\mathbb{R}}$. In particular $\mathbf{x}_{1}^{(i)}, \ldots, \mathbf{x}_{n}^{(i)}$ are linearly independent for each index $i$ with $0 \leq i<s$.

Proof. The second assertion follows from the first because $V_{n}\left(q_{i}\right)=\mathbb{R}^{n}$ for each $i$. We prove the first assertion by induction on $j$. For $j=0$, it is clear since $V_{0}\left(q_{i}\right)=0$ for each $i$. Suppose that it holds for some integer $j$ with $0 \leq j<n$. Fix an arbitrary index $i$ with $0 \leq i<s$. Since $V_{j+1}\left(q_{i}\right)$ has dimension $j+1$ and contains $V_{j}\left(q_{i}\right)$ as a vector subspace of dimension $j$, we are reduced to showing that $\mathbf{x}_{j+1}^{(i)} \in V_{j+1}\left(q_{i}\right) \backslash V_{j}\left(q_{i}\right)$, in order to complete the induction step. If $j<n-1$, we find that

$$
L\left(\mathbf{x}_{j+1}^{(i)}, q_{i}\right) \leq P_{j+1}\left(q_{i}\right)+\epsilon<P_{j+2}\left(q_{i}\right)-\epsilon \leq L_{\mathbf{u}, j+2}\left(q_{i}\right)
$$


where the first inequality comes from (3.3) and (3.4). Thus, we have $\mathbf{x}_{j+1}^{(i)} \in V_{j+1}^{(i)}$ if $j<n-1$. If $j=n-1$, the conclusion is the same because $V_{n}\left(q_{i}\right)=\mathbb{R}^{n}$. So, it remains to show that $\mathbf{x}_{j+1}^{(i)} \notin V_{j}\left(q_{i}\right)$.

Let $S$ be the line segment in $\mathcal{S}$ which contains the point $\left(q_{i}, P_{j+1}\left(q_{i}\right)\right)$ so that $\mathbf{x}_{j+1}^{(i)}=\mathbf{x}_{S}$. If $P_{j+1}$ is constant on $\left[q_{i}, \infty\right)$, then Lemma 3.5 gives $V_{j}\left(q_{i}\right)=V_{j}(\infty)$ and, by construction, we have $\mathbf{x}_{S} \notin V_{j}(\infty)$. We may therefore assume the existence of a largest integer $r$ with $i \leq r<s$ such that $P_{j+1}$ is constant on $\left[q_{i}, q_{r}\right]$. Then the same lemma shows that $V_{j}\left(q_{i}\right)=V_{j}\left(q_{r}\right)$. By construction, we also have $\mathbf{x}_{j+1}^{(i)}=\mathbf{x}_{j+1}^{(r)}=\mathbf{x}_{S}$. Thus, upon replacing $i$ by $r$ if necessary, we may assume that $P_{j+1}$ is non-constant on $\left[q_{i}, q_{i+1}\right]$. This means that $k_{i} \leq j+1 \leq \ell_{i+1}$. If $j+1=k_{i}$, then $\left(q_{i}, P_{j+1}\left(q_{i}\right)\right)$ is the right end-point of $S$ and, by construction, we have $\mathbf{x}_{S} \notin V_{j}\left(q_{i}\right)$. So, we may further assume that $k_{i} \leq j<\ell_{i+1}$. By Lemma 3.7, this implies that $V_{j}\left(q_{i}\right) \neq V_{j}\left(q_{i+1}\right)$. However, by the induction hypothesis, we have

$$
\begin{aligned}
V_{j}\left(q_{i}\right) & =\left\langle\mathbf{x}_{1}^{(i)}, \ldots, \mathbf{x}_{j}^{(i)}\right\rangle_{\mathbb{R}}, \\
V_{j}\left(q_{i+1}\right) & =\left\langle\mathbf{x}_{1}^{(i+1)}, \ldots, \mathbf{x}_{j}^{(i+1)}\right\rangle_{\mathbb{R}}=\left\langle\mathbf{x}_{1}^{(i)}, \ldots, \widehat{\mathbf{x}_{k_{i}}^{(i)}}, \ldots, \mathbf{x}_{j+1}^{(i)}\right\rangle_{\mathbb{R}}
\end{aligned}
$$

where the second expression for $V_{j}\left(q_{i+1}\right)$ comes from (3.2) (if $i+1=s$, the second formula is not part of the induction hypothesis but follows from the definition of the points $\left.\mathbf{x}_{1}^{(s)}, \ldots, \mathbf{x}_{n-1}^{(s)}\right)$. From this, we conclude that $\mathbf{x}_{j+1}^{(i)} \notin V_{j}\left(q_{i}\right)$.

Proof of Property 4). Let $i \in \mathbb{N}$ with $i+1<s$, and put $\ell=\ell_{i+1}$. By Lemma 3.8, we have $\mathbf{x}_{\ell}^{(i+1)} \in V_{\ell}\left(q_{i+1}\right)$, and we simply need to show that $\mathbf{x}_{\ell}^{(i+1)} \in V_{\ell}\left(q_{i}\right)$. If $\ell=n$, this is clear since $V_{n}\left(q_{i}\right)=\mathbb{R}^{n}$. Otherwise, the function $P_{\ell+1}$ is constant on $\left[q_{i}, q_{i+1}\right]$. So, Lemma 3.5 gives $V_{\ell}\left(q_{i}\right)=V_{\ell}\left(q_{i+1}\right)$ and the result follows.

Proof of Property 5). By Lemma 3.8, the integer $\left|\operatorname{det}\left(\mathbf{x}_{1}^{(i)}, \ldots, \mathbf{x}_{n}^{(i)}\right)\right|=\left\|\mathbf{x}_{1}^{(i)} \wedge \cdots \wedge \mathbf{x}_{n}^{(i)}\right\|$ is positive for each $i$. So, its logarithm is bounded below by 0. By Lemma 2.5 together with the estimates (3.3)-(3.4) and the formula (2.7), it is also bounded above by

$$
\log (n)-q_{i}+\sum_{j=1}^{n} L\left(\mathbf{x}_{j}^{(i)}, q_{i}\right) \leq \log (n)-q_{i}+\sum_{j=1}^{n}\left(P_{j}\left(q_{i}\right)+\epsilon\right)=n \epsilon+\log (n) .
$$

Proof of Property 6). Fix an index $i$ with $1 \leq i<s$. We set $k=k_{i}$ and define

$$
\mathbf{y}_{j}=\mathbf{x}_{1}^{(i)} \wedge \cdots \wedge \mathbf{x}_{j}^{(i)} \wedge \cdots \wedge \mathbf{x}_{n}^{(i)} \quad(1 \leq j \leq n) .
$$

Since $\mathbf{y}_{k}, \ldots, \mathbf{y}_{n}$ are $n-k+1$ linearly independent elements of $\bigwedge^{n-1} \mathbb{Z}^{n}$, we have

$$
L_{\mathbf{u}, n-k+1}^{(n-1)}(t) \leq \max \left\{L\left(\mathbf{y}_{k}, t\right), \ldots, L\left(\mathbf{y}_{n}, t\right)\right\} \quad(t \geq 0) .
$$

We use once again the fact that the functions $P_{j}$ with $j \neq k$ are constant on the interval $\left[q_{i}, q_{i}+\delta\right]$, while $P_{k}(t)=P_{k}\left(q_{i}\right)+t-q_{i}$ for each $t \in\left[q_{i}, q_{i}+\delta\right]$. In view of (3.3) and (3.4), this implies that

$$
\left|L\left(\mathbf{x}_{j}^{(i)}, t\right)-P_{j}(t)\right| \leq \epsilon \quad\left(1 \leq j \leq n, q_{i} \leq t \leq q_{i}+\delta\right)
$$


From now on, we restrict to values of $t$ in the smaller interval $\left[q_{i}, q_{i}+\delta / 2\right]$ as this ensures that $P_{j}(t) \geq P_{k}(t)+\delta / 2$ for $j=k+1, \ldots, n$. For each $j=1, \ldots, n$, Lemma 2.4 gives

$$
\begin{aligned}
L\left(\mathbf{y}_{j}, t\right) & \left.\leq \log (n)+L\left(\mathbf{x}_{1}^{(i)}, t\right)+\cdots+\widehat{L\left(\mathbf{x}_{j}^{(i)}, t\right.}\right)+\cdots+L\left(\mathbf{x}_{n}^{(i)}, t\right) \\
& \leq c_{1}+(n-1) \epsilon+P_{1}(t)+\cdots+\widehat{P_{j}(t)}+\cdots+P_{n}(t) \\
& =c_{1}+(n-1) \epsilon+t-P_{j}(t) \quad(\text { by (2.7) }),
\end{aligned}
$$

while Lemma 2.7 (iii) yields

$$
L_{\mathbf{u}, n-k+1}^{(n-1)}(t) \geq t-L_{\mathbf{u}, k}(t)-2 c_{1} \geq t-P_{k}(t)-2 c_{1}-\epsilon .
$$

As $n \epsilon+3 c_{1}<\delta / 2$, this implies that $L_{\mathbf{u}, n-k+1}^{(n-1)}(t)>L\left(\mathbf{y}_{j}, t\right)$ for $j=k+1, \ldots, n$. Therefore, we must have $L_{\mathbf{u}, n-k+1}^{(n-1)}(t) \leq L\left(\mathbf{y}_{k}, t\right)$, which in turn, in view of the above estimates, implies that

$$
t-P_{k}(t)-2 c_{1}-\epsilon \leq L\left(\mathbf{y}_{k}, t\right) \leq c_{1}+(n-1) \epsilon+t-P_{k}(t) \quad\left(q_{i} \leq t \leq q_{i}+\delta / 2\right) .
$$

As the interval $\left[q_{i}, q_{i}+\delta / 2\right]$ has length greater than $3 c_{1}+n \epsilon$ and as the difference $t-P_{k}(t)$ is constant on this interval, the function $L\left(\mathbf{y}_{k}, t\right)$, having slope 0 then 1 , must be constant on $\left[0, q_{i}\right]$ and so we obtain

$$
\log \left\|\mathbf{y}_{k}\right\|=L\left(\mathbf{y}_{k}, q_{i}\right) \geq q_{i}-P_{k}\left(q_{i}\right)-2 c_{1}-\epsilon .
$$

On the other hand, we have

$$
\log \left\|\mathbf{y}_{k}\right\| \leq \sum_{j \neq k} \log \left\|\mathbf{x}_{j}^{(i)}\right\| \leq \sum_{j \neq k}\left(P_{j}\left(q_{i}\right)+\epsilon\right)=(n-1) \epsilon+q_{i}-P_{k}\left(q_{i}\right)
$$

using Property 3) and Formula (2.7). Then 6) follows.

\section{Distance AND height of SUBSPACES}

We gather here definitions and preliminary results that we will need in the next section to deal with the inverse problem to Schmidt's and Summerer's theory.

Let $n \geq 2$ be an integer. We say that a vector subspace $V$ of $\mathbb{R}^{n}$ is defined over $\mathbb{Q}$ if it spanned by elements of $\mathbb{Q}^{n}$. If $V \neq 0$, this is equivalent to asking that $V \cap \mathbb{Z}^{n}$ is a lattice in $V$. Then, following Schmidt in [12], we define the height of $V$, denoted $H(V)$, to be the co-volume of this lattice in $V$. It is given by the formula

$$
H(V)=\left\|\mathbf{x}_{1} \wedge \cdots \wedge \mathbf{x}_{m}\right\|
$$

where $\left(\mathbf{x}_{1}, \ldots, \mathbf{x}_{m}\right)$ is any basis of $V \cap \mathbb{Z}^{n}$, using the natural Euclidean norm on $\bigwedge^{m} \mathbb{R}^{n}$ (see \$2). In particular, we note that $H\left(\mathbb{R}^{n}\right)=1$. We also set $H(0)=1$. The next result is well known.

Lemma 4.1. Let $\left(\mathbf{x}_{1}, \ldots, \mathbf{x}_{n}\right)$ be a basis of $\mathbb{Z}^{n}$ and let $\mathbf{u}$ be a unit vector of $\mathbb{R}^{n}$ perpendicular to $V:=\left\langle\mathbf{x}_{1}, \ldots, \mathbf{x}_{n-1}\right\rangle_{\mathbb{R}}$. Then, we have $H(V)=\left|\mathbf{x}_{n} \cdot \mathbf{u}\right|^{-1}$.

Proof. We find $1=\left\|\mathbf{x}_{1} \wedge \cdots \wedge \mathbf{x}_{n}\right\|=\left\|\mathbf{x}_{1} \wedge \cdots \wedge \mathbf{x}_{n-1}\right\|\left|\mathbf{x}_{n} \cdot \mathbf{u}\right|=H(V)\left|\mathbf{x}_{n} \cdot \mathbf{u}\right|$. 
We define the (projective) distance between two non-zero points $\mathbf{x}$ and $\mathbf{y}$ in $\mathbb{R}^{n}$ by

$$
\operatorname{dist}(\mathbf{x}, \mathbf{y}):=\frac{\|\mathbf{x} \wedge \mathbf{y}\|}{\|\mathbf{x}\|\|\mathbf{y}\|}
$$

It represents the sine of the acute angle between the lines spanned by $\mathbf{x}$ and $\mathbf{y}$ in $\mathbb{R}^{n}$. As a function on $\left(\mathbb{R}^{n} \backslash\{0\}\right)^{2}$, it is continuous and symmetric. It also satisfies the triangle inequality:

$$
\operatorname{dist}(\mathbf{x}, \mathbf{z}) \leq \operatorname{dist}(\mathbf{x}, \mathbf{y})+\operatorname{dist}(\mathbf{y}, \mathbf{z}) \quad\left(\mathbf{x}, \mathbf{y}, \mathbf{z} \in \mathbb{R}^{n} \backslash\{0\}\right) .
$$

For any non-zero point $\mathbf{x} \in \mathbb{R}^{n}$ and any non-zero subspace $V$ of $\mathbb{R}^{n}$, we define the distance from $\mathbf{x}$ to $V$ by

$$
\operatorname{dist}(\mathbf{x}, V):=\inf \{\operatorname{dist}(\mathbf{x}, \mathbf{y}) ; \mathbf{y} \in V \backslash\{0\}\}=\inf \left\{\operatorname{dist}(\mathbf{x}, \mathbf{y}) ; \mathbf{y} \in S^{n} \cap V\right\},
$$

where $S^{n}$ denotes the unit sphere of $\mathbb{R}^{n}$. Since $S^{n} \cap V$ is compact and since dist $(\mathbf{x}, \mathbf{y})$ is a continuous function of $\mathbf{y} \in \mathbb{R}^{n} \backslash\{0\}$, this infimum is in fact a minimum, achieved by at least one point $\mathbf{y}$ in $S^{n} \cap V$. We also define $\operatorname{dist}(\mathbf{x}, 0)=1$ to be consistent with the following result.

Lemma 4.2. Let $\mathbf{x} \in \mathbb{R}^{n} \backslash\{0\}$ and let $V$ be any subspace of $\mathbb{R}^{n}$. Then, we have

$$
\operatorname{dist}(\mathbf{x}, V)=\frac{\left\|\operatorname{proj}_{V^{\perp}}(\mathbf{x})\right\|}{\|\mathbf{x}\|} .
$$

Moreover, for any subspace $U$ of $\mathbb{R}^{n}$ containing $V$, we have $\operatorname{dist}(\mathbf{x}, V) \geq \operatorname{dist}(\mathbf{x}, U)$.

Proof. As the formula gives $\operatorname{dist}(\mathbf{x}, 0)=1$, we may assume that $V \neq 0$. Since $\mathbb{R}^{n}=V \perp V^{\perp}$, we have $\bigwedge^{2} \mathbb{R}^{n}=\bigwedge^{2} V \perp\left(V^{\perp} \wedge V\right) \perp \bigwedge^{2} V^{\perp}$. In particular $\bigwedge^{2} V$ and $V^{\perp} \wedge V$ are orthogonal subspaces of $\mathbb{R}^{n}$. Write $\mathbf{x}=\mathbf{v}+\mathbf{w}$ with $\mathbf{v} \in V$ and $\mathbf{w} \in V^{\perp}$ so that $\mathbf{w}=\operatorname{proj}_{V^{\perp}}(\mathbf{x})$. For any $\mathbf{y} \in V \backslash\{0\}$, we find

$$
\operatorname{dist}(\mathbf{x}, \mathbf{y})=\frac{\|(\mathbf{v} \wedge \mathbf{y})+(\mathbf{w} \wedge \mathbf{y})\|}{\|\mathbf{x}\|\|\mathbf{y}\|} \geq \frac{\|\mathbf{w} \wedge \mathbf{y}\|}{\|\mathbf{x}\|\|\mathbf{y}\|}=\frac{\|\mathbf{w}\|}{\|\mathbf{x}\|}
$$

with equality if and only if $\mathbf{v} \wedge \mathbf{y}=0$. As there exists $\mathbf{y} \in V \backslash\{0\}$ satisfying the latter condition, this proves the first assertion. The second one is clear.

Finally, given non-zero subspaces $V_{1}$ and $V_{2}$ of $\mathbb{R}^{n}$, we define the distance from $V_{1}$ to $V_{2}$ by

$$
\operatorname{dist}\left(V_{1}, V_{2}\right):=\sup \left\{\operatorname{dist}\left(\mathbf{x}, V_{2}\right) ; \mathbf{x} \in V_{1} \backslash\{0\}\right\}=\sup \left\{\operatorname{dist}\left(\mathbf{x}, V_{2}\right) ; \mathbf{x} \in S^{n} \cap V_{1}\right\},
$$

Since $S^{n} \cap V_{1}$ is compact and since the above lemma shows that $\operatorname{dist}\left(\mathbf{x}, V_{2}\right)$ is a continuous function of $\mathbf{x}$ on $\mathbb{R}^{n} \backslash\{0\}$, this supremum is also achieved by some point $\mathbf{x} \in S^{n} \cap V_{1}$. Note that this distance is not, in general, a symmetric function of $V_{1}$ and $V_{2}$. However, it satisfies the following property.

Lemma 4.3. Let $\mathbf{x} \in \mathbb{R}^{n} \backslash\{0\}$ and let $V_{1}, V_{2}$ be non-zero subspaces of $\mathbb{R}^{n}$. Then, we have

$$
\operatorname{dist}\left(\mathbf{x}, V_{2}\right) \leq \operatorname{dist}\left(\mathbf{x}, V_{1}\right)+\operatorname{dist}\left(V_{1}, V_{2}\right) \text {. }
$$

Moreover, if $V$ is also a non-zero subspace of $\mathbb{R}^{n}$, then

$$
\operatorname{dist}\left(V, V_{2}\right) \leq \operatorname{dist}\left(V, V_{1}\right)+\operatorname{dist}\left(V_{1}, V_{2}\right) .
$$


Proof. Choose $\mathbf{y} \in S^{n} \cap V_{1}$ such that $\operatorname{dist}\left(\mathbf{x}, V_{1}\right)=\operatorname{dist}(\mathbf{x}, \mathbf{y})$ and $\mathbf{z} \in S^{n} \cap V_{2}$ such that $\operatorname{dist}\left(\mathbf{y}, V_{2}\right)=\operatorname{dist}(\mathbf{y}, \mathbf{z})$. Then

$$
\begin{aligned}
\operatorname{dist}\left(\mathbf{x}, V_{2}\right) \leq \operatorname{dist}(\mathbf{x}, \mathbf{z}) & \leq \operatorname{dist}(\mathbf{x}, \mathbf{y})+\operatorname{dist}(\mathbf{y}, \mathbf{z}) \\
& =\operatorname{dist}\left(\mathbf{x}, V_{1}\right)+\operatorname{dist}\left(\mathbf{y}, V_{2}\right) \leq \operatorname{dist}\left(\mathbf{x}, V_{1}\right)+\operatorname{dist}\left(V_{1}, V_{2}\right) .
\end{aligned}
$$

The second inequality follows from this by choosing $\mathbf{x} \in V \operatorname{such}$ that $\operatorname{dist}\left(V, V_{2}\right)=\operatorname{dist}\left(\mathbf{x}, V_{2}\right)$ and then using $\operatorname{dist}\left(\mathbf{x}, V_{1}\right) \leq \operatorname{dist}\left(V, V_{1}\right)$.

The next lemma shows a case where the distance between two vector subspaces is a symmetric function of the subspaces.

Lemma 4.4. Let $V_{1}, V_{2}$ be non-zero subspaces of co-dimension 1 inside a subspace $U$ of $\mathbb{R}^{n}$. For $i=1,2$, choose a unit vector $\mathbf{u}_{i}$ in $U \cap V_{i}^{\perp}$. Then, we have

$$
\operatorname{dist}\left(V_{1}, V_{2}\right)=\operatorname{dist}\left(\mathbf{u}_{1}, \mathbf{u}_{2}\right) \text {. }
$$

Proof. For the first assertion, we may assume that $V_{1} \neq V_{2}$ because otherwise $\operatorname{dist}\left(V_{1}, V_{2}\right)=$ $0=\operatorname{dist}\left(\mathbf{u}_{1}, \mathbf{u}_{2}\right)$. Under this hypothesis, we define $W:=V_{1} \cap V_{2}$ and, for $j=1,2$, we choose a unit vector $\mathbf{v}_{j}$ in $V_{j} \cap W^{\perp}$. Then $\left(\mathbf{u}_{1}, \mathbf{v}_{1}\right)$ and $\left(\mathbf{u}_{2}, \mathbf{v}_{2}\right)$ are two orthonormal bases of $U \cap W^{\perp}$ and so

$$
\operatorname{dist}\left(\mathbf{u}_{1}, \mathbf{u}_{2}\right)=\left\|\mathbf{u}_{1} \wedge \mathbf{u}_{2}\right\|=\left|\mathbf{v}_{1} \cdot \mathbf{u}_{2}\right|
$$

Let $\mathbf{x}$ be a non-zero element of $V_{1}$. Upon writing $\mathbf{x}=\mathbf{w}+a \mathbf{v}_{1}$ with $\mathbf{w} \in W$ and $a \in \mathbb{R}$, we find, using Lemma 4.2 ,

$$
\operatorname{dist}\left(\mathbf{x}, V_{2}\right)=\frac{\left\|\operatorname{proj}_{V_{2}^{\perp}}\left(\mathbf{w}+a \mathbf{v}_{1}\right)\right\|}{\left\|\mathbf{w}+a \mathbf{v}_{1}\right\|}=\frac{|a|\left|\mathbf{v}_{1} \cdot \mathbf{u}_{2}\right|}{\left\|\mathbf{w}+a \mathbf{v}_{1}\right\|} \leq\left|\mathbf{v}_{1} \cdot \mathbf{u}_{2}\right|
$$

with equality if and only if $\mathbf{w}=0$. Thus, $\operatorname{dist}\left(V_{1}, V_{2}\right)=\left|\mathbf{v}_{1} \cdot \mathbf{u}_{2}\right|=\operatorname{dist}\left(\mathbf{u}_{1}, \mathbf{u}_{2}\right)$.

Definition 4.5. We say that a non-empty sequence $\left(\mathbf{x}_{1}, \ldots, \mathbf{x}_{m}\right)$ of vectors of $\mathbb{R}^{n}$ is almost orthogonal if it is linearly independent and satisfies

$$
\operatorname{dist}\left(\mathbf{x}_{j},\left\langle\mathbf{x}_{1}, \ldots, \mathbf{x}_{j-1}\right\rangle_{\mathbb{R}}\right) \geq 1-\frac{1}{2^{j-1}} \quad(2 \leq j \leq m) .
$$

Thus, any sequence $(\mathbf{x})$ consisting of just one non-zero vector $\mathbf{x} \in \mathbb{R}^{n}$ is almost orthogonal. By Lemma 4.2, it follows that any non-empty subsequence of an almost orthogonal sequence is almost orthogonal. We conclude this section with two more facts linked with this notion.

Lemma 4.6. Let $\left(\mathbf{x}_{1}, \ldots, \mathbf{x}_{m}\right)$ be an almost orthogonal primitive $m$-tuple of points of $\mathbb{Z}^{n}$ and let $U:=\left\langle\mathbf{x}_{1}, \ldots, \mathbf{x}_{m}\right\rangle_{\mathbb{R}}$. Then, we have

$$
e^{-2}\left\|\mathbf{x}_{1}\right\| \cdots\left\|\mathbf{x}_{m}\right\| \leq H(U) \leq\left\|\mathbf{x}_{1}\right\| \cdots\left\|\mathbf{x}_{m}\right\|
$$

Proof. Since $H(U)=\left\|\mathbf{x}_{1} \wedge \cdots \wedge \mathbf{x}_{m}\right\|$, the upper bound is clear. For the lower bound, we use induction to show that

$$
\left\|\mathbf{x}_{1} \wedge \cdots \wedge \mathbf{x}_{k}\right\| \geq\left\|\mathbf{x}_{1}\right\| \cdots\left\|\mathbf{x}_{k}\right\| \prod_{j=2}^{k}\left(1-2^{1-j}\right) \quad(1 \leq k \leq m) .
$$


Since $\prod_{j=2}^{m}\left(1-2^{1-j}\right) \geq \prod_{j=2}^{\infty} \exp \left(-2^{2-j}\right)=e^{-2}$, this will complete the proof. For $k=1$, the inequality (4.1) is clear. Suppose that it holds for some integer $k$ with $1 \leq k<m$. Set $V=\left\langle\mathbf{x}_{1}, \ldots, \mathbf{x}_{k}\right\rangle_{\mathbb{R}}$. Using Lemma 4.2, we find

$$
\begin{aligned}
\left\|\mathbf{x}_{1} \wedge \cdots \wedge \mathbf{x}_{k+1}\right\| & =\left\|\mathbf{x}_{1} \wedge \cdots \wedge \mathbf{x}_{k}\right\|\left\|\operatorname{proj}_{V^{\perp}}\left(\mathbf{x}_{k+1}\right)\right\| \\
& =\left\|\mathbf{x}_{1} \wedge \cdots \wedge \mathbf{x}_{k}\right\|\left\|\mathbf{x}_{k+1}\right\| \operatorname{dist}\left(\mathbf{x}_{k+1}, V\right) \\
& =\left\|\mathbf{x}_{1} \wedge \cdots \wedge \mathbf{x}_{k}\right\|\left\|\mathbf{x}_{k+1}\right\|\left(1-2^{-k}\right) .
\end{aligned}
$$

So, (4.1) also holds with $k$ replaced by $k+1$.

Lemma 4.7. Let $k, \ell, m \in\{1, \ldots, n\}$ with $k<\ell \leq m$, let $U$ be a subspace of $\mathbb{R}^{n}$ of dimension $m$ defined over $\mathbb{Q}$, and let $\left(\mathbf{y}_{1}, \ldots, \mathbf{y}_{m}\right)$ be a basis of $U \cap \mathbb{Z}^{n}$. Define

$$
V_{1}=\left\langle\mathbf{y}_{1}, \ldots, \widehat{\mathbf{y}_{\ell}}, \ldots, \mathbf{y}_{m}\right\rangle_{\mathbb{R}} \text { and } V_{2}=\left\langle\mathbf{y}_{1}, \ldots, \widehat{\mathbf{y}_{k}}, \ldots, \mathbf{y}_{m}\right\rangle_{\mathbb{R}}
$$

Then, we have

$$
\operatorname{dist}\left(V_{1}, V_{2}\right)=\frac{H\left(V_{1} \cap V_{2}\right) H(U)}{H\left(V_{1}\right) H\left(V_{2}\right)} .
$$

Moreover, if $\left(\mathbf{y}_{1}, \ldots, \widehat{\mathbf{y}_{\ell}}, \ldots, \mathbf{y}_{m}\right)$ and $\left(\mathbf{y}_{1}, \ldots, \widehat{\mathbf{y}_{k}}, \ldots, \mathbf{y}_{m}\right)$ are almost orthogonal, then

$$
\operatorname{dist}\left(V_{1}, V_{2}\right) \leq \frac{e^{4} H(U)}{\left\|\mathbf{y}_{1}\right\| \cdots\left\|\mathbf{y}_{m}\right\|}
$$

Proof. Set $W=V_{1} \cap V_{2}=\left\langle\mathbf{y}_{1} \wedge \cdots \wedge \widehat{\mathbf{y}_{k}} \wedge \cdots \wedge \widehat{\mathbf{y}_{\ell}} \wedge \cdots \wedge \mathbf{y}_{m}\right\rangle_{\mathbb{R}}$. For $j=1,2$, we choose unit vectors $\mathbf{u}_{j}$ in $U \cap V_{j}^{\perp}$ and $\mathbf{v}_{j}$ in $V_{j} \cap W^{\perp}$. Then $\left(\mathbf{u}_{1}, \mathbf{v}_{1}\right)$ and $\left(\mathbf{u}_{2}, \mathbf{v}_{2}\right)$ are orthonormal bases of $U \cap W^{\perp}$. So, $\mathbf{v}_{1} \wedge \mathbf{v}_{2}= \pm \mathbf{u}_{1} \wedge \mathbf{u}_{2}$ and, by Lemma 4.4, we obtain $\left\|\mathbf{v}_{1} \wedge \mathbf{v}_{2}\right\|=\operatorname{dist}\left(V_{1}, V_{2}\right)$.

Define $\omega=\mathbf{y}_{1} \wedge \cdots \wedge \widehat{\mathbf{y}_{k}} \wedge \cdots \wedge \widehat{\mathbf{y}_{\ell}} \wedge \cdots \wedge \mathbf{y}_{m}$, so that $H(W)=\|\omega\|$. Upon writing $\mathbf{y}_{k}=\mathbf{w}_{1}+a_{1} \mathbf{v}_{1}$ and $\mathbf{y}_{\ell}=\mathbf{w}_{2}+a_{2} \mathbf{v}_{2}$ with $\mathbf{w}_{1}, \mathbf{w}_{2} \in W$ and $a_{1}, a_{2} \in \mathbb{R}$, we find

$$
\begin{aligned}
H\left(V_{j}\right) & =\left\|\omega \wedge\left(\mathbf{w}_{j}+a_{j} \mathbf{v}_{j}\right)\right\|=\|\omega\|\left|a_{j}\right| \quad(j=1,2), \\
H(U) & =\left\|\omega \wedge\left(\mathbf{w}_{1}+a_{1} \mathbf{v}_{1}\right) \wedge\left(\mathbf{w}_{2}+a_{2} \mathbf{v}_{2}\right)\right\| \\
& =\|\omega\|\left\|a_{1} a_{2} \mathbf{v}_{1} \wedge \mathbf{v}_{2}\right\|=\|\omega\|\left|a_{1} a_{2}\right| \operatorname{dist}\left(V_{1}, V_{2}\right),
\end{aligned}
$$

and (4.2) follows because $a_{1} a_{2} \neq 0$.

If $\left(\mathbf{x}_{1}, \ldots, \widehat{\mathbf{x}_{\ell}}, \ldots, \mathbf{x}_{m}\right)$ and $\left(\mathbf{y}_{1}, \ldots, \widehat{\mathbf{y}_{k}}, \ldots, \mathbf{y}_{m}\right)$ are almost orthogonal, Lemma 4.6 gives

$$
H\left(V_{1}\right) \geq \frac{\left\|\mathbf{y}_{1}\right\| \ldots\left\|\mathbf{y}_{m}\right\|}{e^{2}\left\|\mathbf{y}_{\ell}\right\|} \quad \text { and } \quad H\left(V_{2}\right) \geq \frac{\left\|\mathbf{y}_{1}\right\| \ldots\left\|\mathbf{y}_{m}\right\|}{e^{2}\left\|\mathbf{y}_{k}\right\|} .
$$

Then (4.3) follows from (4.2) using $H(W)=\|\omega\| \leq\left(\left\|\mathbf{y}_{1}\right\| \ldots\left\|\mathbf{y}_{m}\right\|\right) /\left(\left\|\mathbf{y}_{k}\right\|\left\|\mathbf{y}_{\ell}\right\|\right)$.

\section{THE INVERSE PROBLEM FOR RIGID SYSTEMS}

The goal of this section is to prove a partial converse to Schmidt's and Summerer's theorem 2.9 for rigid systems with large mesh. Motivated by Theorem 3.1, we start by constructing recursively a sequence of bases of $\mathbb{Z}^{n}$ with several strong properties. The key to the recurrence is provided by the following lemma. 
Lemma 5.1. Let $h, k, \ell \in\{1, \ldots, n\}$ with $h \leq \ell$ and $k<\ell$, let $\left(\mathbf{x}_{1}, \ldots, \mathbf{x}_{n}\right)$ be a basis of $\mathbb{Z}^{n}$, and let $A$ be a real number with $A \geq 2^{\ell}\left(\left\|\mathbf{x}_{1}\right\|+\cdots+\left\|\mathbf{x}_{\ell}\right\|\right)$. Then, there exists a basis $\left(\mathbf{y}_{1}, \ldots, \mathbf{y}_{n}\right)$ of $\mathbb{Z}^{n}$ satisfying

1) $\left(\mathbf{y}_{1}, \ldots, \widehat{\mathbf{y}_{\ell}}, \ldots, \mathbf{y}_{n}\right)=\left(\mathbf{x}_{1}, \ldots, \widehat{\mathbf{x}_{h}}, \ldots, \mathbf{x}_{n}\right)$

2) $\mathbf{y}_{\ell} \in \mathbf{x}_{h}+\left\langle\mathbf{x}_{1}, \ldots, \widehat{\mathbf{x}_{h}}, \ldots, \mathbf{x}_{\ell}\right\rangle_{\mathbb{Z}}$,

3) $A \leq\left\|\mathbf{y}_{\ell}\right\| \leq 2 A$,

4) $\operatorname{dist}\left(\mathbf{y}_{\ell},\left\langle\mathbf{y}_{1}, \ldots, \widehat{\mathbf{y}_{k}}, \ldots, \mathbf{y}_{\ell-1}\right\rangle_{\mathbb{R}}\right) \geq 1-\frac{1}{2^{\ell-1}}$.

Moreover, if $\left(\mathbf{x}_{1}, \ldots, \widehat{\mathbf{x}_{h}}, \ldots, \mathbf{x}_{\ell}\right)$ is almost orthogonal, then $\left(\mathbf{y}_{1}, \ldots, \widehat{\mathbf{y}_{k}}, \ldots, \mathbf{y}_{\ell}\right)$ is almost orthogonal.

Note that the condition 4) is stronger than needed in order to establish that the sequence $\left(\mathbf{y}_{1}, \ldots, \widehat{\mathbf{y}_{k}}, \ldots, \mathbf{y}_{\ell}\right)$ is almost orthogonal. In view of the definition, a lower bound of $1-1 / 2^{\ell-2}$ would suffice instead of $1-1 / 2^{\ell-1}$. However, this stronger requirement will show its importance later. Note also that 4 ) trivially holds when $\ell=2$.

Proof. We use 1) as a definition of the vectors $\mathbf{y}_{1}, \ldots, \widehat{\mathbf{y}}_{\ell}, \ldots, \mathbf{y}_{n}$. Then, $\left(\mathbf{y}_{1}, \ldots, \mathbf{y}_{n}\right)$ is a basis of $\mathbb{Z}^{n}$ for any choice of $\mathbf{y}_{\ell}$ in $\mathbf{x}_{h}+\left\langle\mathbf{x}_{1}, \ldots, \widehat{\mathbf{x}_{h}}, \ldots, \mathbf{x}_{n}\right\rangle_{\mathbb{Z}}$. A fortiori, it is a basis of $\mathbb{Z}^{n}$ for any choice of $\mathbf{y}_{\ell}$ satisfying 2). To prove the existence of a point $\mathbf{y}_{\ell}$ satisfying 2 ), 3) and 4 ), we first observe that the hypothesis $h \leq \ell$ yields

$$
\left(\mathbf{y}_{1}, \ldots, \mathbf{y}_{\ell-1}\right)=\left(\mathbf{x}_{1}, \ldots, \widehat{\mathbf{x}_{h}}, \ldots, \mathbf{x}_{\ell}\right) .
$$

Consider the chain of subspaces $W \subset V \subset U$ of relative codimension 1 given by

$$
\begin{aligned}
W & =\left\langle\mathbf{y}_{1}, \ldots, \widehat{\mathbf{y}_{k}}, \ldots, \mathbf{y}_{\ell-1}\right\rangle_{\mathbb{R}}, \\
V & =\left\langle\mathbf{y}_{1}, \ldots, \mathbf{y}_{\ell-1}\right\rangle_{\mathbb{R}}=\left\langle\mathbf{x}_{1}, \ldots, \widehat{\mathbf{x}_{h}}, \ldots, \mathbf{x}_{\ell}\right\rangle_{\mathbb{R}}, \\
U & =\left\langle\mathbf{x}_{1}, \ldots, \mathbf{x}_{\ell}\right\rangle_{\mathbb{R}} .
\end{aligned}
$$

Choose unit vectors $\mathbf{u} \in U \cap V^{\perp}$ and $\mathbf{v} \in V \cap W^{\perp}$. Then $\{\mathbf{u}, \mathbf{v}\}$ is an orthonormal basis of $U \cap W^{\perp}$ and we have $\operatorname{proj}_{V^{\perp}}\left(\mathbf{x}_{h}\right)=c \mathbf{u}$ where $c=\mathbf{x}_{h} \cdot \mathbf{u}$. Put $B=(3 / 2) A$. Since the vector $c \mathbf{u}+B \mathbf{v}-\mathbf{x}_{h}$ belongs to $V$, we can write

$$
c \mathbf{u}+B \mathbf{v}=\mathbf{x}_{h}+\sum_{j=1}^{\ell-1} c_{j} \mathbf{y}_{j}
$$

for some $c_{1}, \ldots, c_{\ell-1} \in \mathbb{R}$. We choose

$$
\mathbf{y}_{\ell}:=\mathbf{x}_{h}+\sum_{j=1}^{\ell-1}\left\lceil c_{j}\right\rceil \mathbf{y}_{j}=c \mathbf{u}+B \mathbf{v}+\sum_{j=1}^{\ell-1} \epsilon_{j} \mathbf{y}_{j}
$$

where $\epsilon_{j}=\left\lceil c_{j}\right\rceil-c_{j} \in[0,1)$ for $j=1, \ldots, \ell-1$. Then, the condition 2) is fulfilled and

$$
\left|\left\|\mathbf{y}_{\ell}\right\|-B\right| \leq\left\|\mathbf{y}_{\ell}-B \mathbf{v}\right\| \leq|c|+\sum_{j=1}^{\ell-1}\left\|\mathbf{y}_{j}\right\| \leq \sum_{j=1}^{\ell}\left\|\mathbf{x}_{j}\right\| \leq \frac{B}{2^{\ell}}
$$


so that $\left(1-2^{-\ell}\right) B \leq\left\|\mathbf{y}_{\ell}\right\| \leq\left(1+2^{-\ell}\right) B$. Since $\ell \geq 2$, this shows that 3$)$ holds. Moreover, we find that

$$
\left\|\operatorname{proj}_{W^{\perp}}\left(\mathbf{y}_{\ell}\right)\right\| \geq\left|\mathbf{y}_{\ell} \cdot \mathbf{v}\right|=\left|B+\epsilon_{k} \mathbf{y}_{k} \cdot \mathbf{v}\right| \geq B-\left\|\mathbf{y}_{k}\right\| \geq\left(1-2^{-\ell}\right) B
$$

and therefore

$$
\operatorname{dist}\left(\mathbf{y}_{\ell}, W\right)=\frac{\left\|\operatorname{proj}_{W^{\perp}}\left(\mathbf{y}_{\ell}\right)\right\|}{\left\|\mathbf{y}_{\ell}\right\|} \geq \frac{1-2^{-\ell}}{1+2^{-\ell}} \geq 1-\frac{1}{2^{\ell-1}} .
$$

Thus $\mathbf{y}_{\ell}$ also satisfies 4$)$. Finally, if $\left(\mathbf{x}_{1}, \ldots, \widehat{\mathbf{x}_{h}}, \ldots, \mathbf{x}_{\ell}\right)=\left(\mathbf{y}_{1}, \ldots, \mathbf{y}_{\ell-1}\right)$ is almost orthogonal, then its subsequence $\left(\mathbf{y}_{1}, \ldots, \widehat{\mathbf{y}_{k}}, \ldots, \mathbf{y}_{\ell-1}\right)$ is almost orthogonal, and by 4$)$ we conclude that $\left(\mathbf{y}_{1}, \ldots, \widehat{\mathbf{y}_{k}}, \ldots, \mathbf{y}_{\ell}\right)$ is almost orthogonal.

The next lemma provides us with an initial basis of $\mathbb{Z}^{n}$ to start the recurrence.

Lemma 5.2. Let $k$ be an integer with $1 \leq k \leq n$ and let $A_{1}, \ldots, A_{n}$ be real numbers satisfying $A_{1} \geq 8$ and $A_{j} \geq 2^{j+3} A_{j-1}$ for $j=2, \ldots, n$. Then there exists a basis $\left(\mathbf{y}_{1}, \ldots, \mathbf{y}_{n}\right)$ of $\mathbb{Z}^{n}$ which satisfies the following conditions

(i) $\left(\mathbf{y}_{1}, \ldots, \mathbf{y}_{n-1}\right)$ and $\left(\mathbf{y}_{1}, \ldots, \widehat{\mathbf{y}_{k}}, \ldots, \mathbf{y}_{n}\right)$ are almost orthogonal,

(ii) $A_{j} \leq\left\|\mathbf{y}_{j}\right\| \leq 2 A_{j}$ for $j=1, \ldots, n$,

(iii) $\operatorname{dist}\left(\mathbf{y}_{n},\left\langle\mathbf{y}_{1}, \ldots, \widehat{\mathbf{y}_{k}}, \ldots, \mathbf{y}_{n-1}\right\rangle_{\mathbb{R}}\right) \geq 1-1 / 2^{n-1}$ if $k<n$.

Note that, like in the preceding lemma, the lower bound $1-1 / 2^{n-1}$ imposed in condition (iii) is larger than the lower bound $1-1 / 2^{n-2}$ which follows from condition (i) if $k<n$.

Proof. Let $\left(\mathbf{e}_{1}, \ldots, \mathbf{e}_{n}\right)$ denote the canonical basis of $\mathbb{Z}^{n}$. Lemma 5.1 allows us to construct recursively a sequence of integer points $\mathbf{x}_{1}, \ldots, \mathbf{x}_{n} \in \mathbb{Z}^{n}$ starting with $\mathbf{x}_{1}=\mathbf{e}_{1}$ which, for each $j=2, \ldots, n$, satisfy the following properties:

1) $\left(\mathbf{x}_{1}, \ldots, \mathbf{x}_{j-1}, \mathbf{e}_{j}, \ldots, \mathbf{e}_{n}\right)$ is a basis of $\mathbb{Z}^{n}$,

2) $\mathbf{x}_{j} \in \mathbf{e}_{j}+\left\langle\mathbf{x}_{1}, \ldots, \mathbf{x}_{j-1}\right\rangle_{\mathbb{Z}}$

3) $A_{j-1} \leq\left\|\mathbf{x}_{j}\right\| \leq 2 A_{j-1}$,

4) $\operatorname{dist}\left(\mathbf{x}_{j},\left\langle\mathbf{x}_{2}, \ldots, \mathbf{x}_{j-1}\right\rangle_{\mathbb{R}}\right) \geq 1-1 / 2^{j-1}$ if $j \geq 3$.

Indeed, the condition 1) holds for $j=2$ because $\mathbf{x}_{1}=\mathbf{e}_{1}$. Suppose that we have constructed $\mathbf{x}_{1}, \ldots, \mathbf{x}_{\ell-1}$ for some $\ell$ with $2 \leq \ell \leq n$ so that 1 ) holds when $2 \leq j \leq \ell$ while 2), 3) and 4) hold when $2 \leq j \leq \ell-1$. We apply Lemma 5.1 to the basis $\left(\mathbf{x}_{1}, \ldots, \mathbf{x}_{\ell-1}, \mathbf{e}_{\ell}, \ldots, \mathbf{e}_{n}\right)$ of $\mathbb{Z}^{n}$ with the choice of $h=\ell, k=1$ and $A=A_{\ell-1}$. Since

$$
2^{\ell}\left(\left\|\mathbf{x}_{1}\right\|+\cdots+\left\|\mathbf{x}_{\ell-1}\right\|+\left\|\mathbf{e}_{\ell}\right\|\right) \leq 2^{\ell+1}\left(1+\sum_{2 \leq j \leq \ell-1} A_{j-1}\right) \leq A_{\ell-1},
$$

it provides a new point $\mathbf{x}_{\ell}$ of $\mathbb{Z}^{n}$ which satisfies the condition 1) for $j=\ell+1$ as well as the conditions 2),3) and 4) for $j=\ell$. This proves our claim and, in the case $\ell=n$, shows that $\left(\mathbf{x}_{1}, \ldots, \mathbf{x}_{n}\right)$ is a basis of $\mathbb{Z}^{n}$. Moreover, since 4$)$ holds for $j=3, \ldots, n$, we also note that $\left(\mathbf{x}_{2}, \ldots, \mathbf{x}_{n}\right)$ is almost orthogonal.

If $k<n$, we apply once again Lemma [5.1, this time to the basis $\left(\mathbf{x}_{1}, \ldots, \mathbf{x}_{n}\right)$ with the choice of $h=1, \ell=n$ and the given value of $k$. Since

$$
2^{n}\left(\left\|\mathbf{x}_{1}\right\|+\cdots+\left\|\mathbf{x}_{n}\right\|\right) \leq 2^{n+1}\left(1+\sum_{2 \leq j \leq n} A_{j-1}\right) \leq A_{n}
$$


it provides a new basis of $\mathbb{Z}^{n}$ of the form $\left(\mathbf{y}_{1}, \ldots, \mathbf{y}_{n}\right)=\left(\mathbf{x}_{2}, \ldots, \mathbf{x}_{n}, \mathbf{y}_{n}\right)$ with all the requested properties. Otherwise, it suffices to choose $\left(\mathbf{y}_{1}, \ldots, \mathbf{y}_{n}\right)=\left(\mathbf{x}_{2}, \ldots, \mathbf{x}_{n}, \mathbf{x}_{1}+B \mathbf{x}_{2}\right)$ with $B=$ $\left\lceil A_{n} /\left\|\mathbf{x}_{2}\right\|\right\rceil+1$.

From now on, we fix a choice of $s \in \mathbb{N}^{*} \cup\{\infty\}$ and put

$$
C=2^{n+3} e^{4} \text {. }
$$

We suppose that, for each integer $i$ with $0 \leq i<s$, we are given a point $\mathbf{A}^{(i)}=\left(A_{1}^{(i)}, \ldots, A_{n}^{(i)}\right)$ in $\mathbb{R}^{n}$ and integers $k_{i}$ and $\ell_{i}$ satisfying the conditions

$$
\begin{aligned}
& 1 \leq k \leq \ell_{0}=n \quad \text { and } 1 \leq k_{i}<\ell_{i} \leq n \quad \text { if } i \geq 1 \\
& A_{1}^{(i)} \geq C, \quad A_{j}^{(i)} \geq A_{j-1}^{(i)} C \text { for } j=2, \ldots, n \\
& k_{i-1} \leq \ell_{i} \text { and } A_{\ell_{i}}^{(i)} \geq A_{\ell_{i}}^{(i-1)} C \quad \text { if } i \geq 1 \\
& \left(A_{1}^{(i)}, \ldots, \widehat{A_{\ell_{i}}^{(i)}}, \ldots, A_{n}^{(i)}\right)=\left(A_{1}^{(i-1)}, \ldots, \widehat{A_{k_{i-1}}^{(i-1)}}, \ldots, A_{n}^{(i-1)}\right) \quad \text { if } i \geq 1 .
\end{aligned}
$$

We now combine the previous lemmas to establish the following result.

Proposition 5.3. For each integer $i$ with $0 \leq i<s$, there exists a basis $\left(\mathbf{x}_{1}^{(i)}, \ldots, \mathbf{x}_{n}^{(i)}\right)$ of $\mathbb{Z}^{n}$ which satisfies the following properties:
1) $\left(\mathbf{x}_{1}^{(0)}, \ldots, \mathbf{x}_{n-1}^{(0)}\right)$ is almost orthogonal,
2) $\left(\mathbf{x}_{1}^{(i)}, \ldots, \widehat{\mathbf{x}_{k_{i}}^{(i)}}, \ldots, \mathbf{x}_{n}^{(i)}\right)$ is almost orthogonal,
3) $A_{j}^{(i)} \leq\left\|\mathbf{x}_{j}^{(i)}\right\| \leq 2 A_{j}^{(i)}$ for $j=1, \ldots, n$,
4) $\operatorname{dist}\left(\mathbf{x}_{\ell_{i}}^{(i)},\left\langle\mathbf{x}_{1}^{(i)}, \ldots, \widehat{\mathbf{x}_{k_{i}}^{(i)}}, \ldots, \mathbf{x}_{\ell_{i}-1}^{(i)}\right\rangle_{\mathbb{R}}\right) \geq 1-\frac{1}{2^{\ell_{i}-1}}$ if $k_{i}<\ell_{i}$,
5) $\mathbf{x}_{\ell_{i}}^{(i)} \in \mathbf{x}_{k_{i-1}}^{(i-1)}+\left\langle\mathbf{x}_{1}^{(i-1)}, \ldots, \widehat{\mathbf{x}_{k_{i-1}}^{(i-1)}}, \ldots, \mathbf{x}_{\ell_{i}}^{(i-1)}\right\rangle_{\mathbb{Z}}$ if $i \geq 1$,
6) $\left(\mathbf{x}_{1}^{(i)}, \ldots, \widehat{\mathbf{x}_{\ell_{i}}^{(i)}}, \ldots, \mathbf{x}_{n}^{(i)}\right)=\left(\mathbf{x}_{1}^{(i-1)}, \ldots, \widehat{\mathbf{x}_{k_{i-1}}^{(i-1)}}, \ldots, \mathbf{x}_{n}^{(i-1)}\right)$ if $i \geq 1$.

Moreover, if $\mathbf{u}_{i}$ denotes a unit vector orthogonal to $\left\langle\mathbf{x}_{1}^{(i)}, \ldots, \widehat{\mathbf{x}_{k_{i}}^{(i)}}, \ldots, \mathbf{x}_{n}^{(i)}\right\rangle_{\mathbb{R}}$ then, for each pair of integers $i$ and $j$ with $0 \leq i<j<s$, we have

$$
\operatorname{dist}\left(\mathbf{u}_{i}, \mathbf{u}_{j}\right) \leq \frac{2 e^{4}}{\left\|\mathbf{x}_{1}^{(i+1)}\right\| \cdots\left\|\mathbf{x}_{n}^{(i+1)}\right\|}
$$

Proof. We prove the first assertion by induction on $i$. To construct $\left(\mathbf{x}_{1}^{(0)}, \ldots, \mathbf{x}_{n}^{(0)}\right)$, we apply Lemma 5.2 with the choice of $k=k_{0}$ and $A_{j}=A_{j}^{(0)}$ for $j=1, \ldots, n$. Thanks to (5.2), the hypotheses of the lemma are satisfied and, since $\ell_{0}=n$, the basis that we obtain satisfies the conditions 1 ) to 4 ) of the proposition for $i=0$. The conditions 5) and 6) are vacuous.

Now, let $t$ be an integer with $1 \leq t<s$. Suppose that, for $i=0, \ldots, t-1$, we have constructed a basis $\left(\mathbf{x}_{1}^{(i)}, \ldots, \mathbf{x}_{n}^{(i)}\right)$ of $\mathbb{Z}^{n}$ which satisfies all conditions 1$)$ to 6$)$. To construct the next basis, we apply Lemma 5.1 to $\left(\mathbf{x}_{1}^{(t-1)}, \ldots, \mathbf{x}_{n}^{(t-1)}\right)$ for the choice of $h=k_{t-1}, k=k_{t}$, $\ell=\ell_{t}$ and $A=A_{\ell_{t}}^{(t)}$. Since 3) holds for $i=t-1$, we find with the help of (5.2) and (5.3) that

$$
2^{\ell_{t}}\left(\left\|\mathbf{x}_{1}^{(t-1)}\right\|+\cdots+\left\|\mathbf{x}_{\ell_{t}}^{(t-1)}\right\|\right) \leq 2^{\ell_{t}+1}\left(A_{1}^{(t-1)}+\cdots+A_{\ell_{t}}^{(t-1)}\right) \leq 2^{\ell_{t}+2} A_{\ell_{t}}^{(t-1)} \leq A_{\ell_{t}}^{(t)}
$$


So, the lemma produces a new basis $\left(\mathbf{x}_{1}^{(t)}, \ldots, \mathbf{x}_{n}^{(t)}\right)$ of $\mathbb{Z}^{n}$ which satisfies the conditions 4$)$ to 6) for $i=t$, as well as $A_{\ell_{t}}^{(t)} \leq\left\|\mathbf{x}_{\ell_{t}}^{(t)}\right\| \leq 2 A_{\ell_{t}}^{(t)}$. Then, combining our hypothesis that 3 ) holds for $i=t-1$ with the equalities 6) and (5.4) for $i=t$, we conclude that 3) holds for $i=t$.

To complete the inductive step, it remains to show that $\left(\mathbf{x}_{1}^{(t)}, \ldots, \widehat{\mathbf{x}_{k t}^{(t)}}, \ldots, \mathbf{x}_{n}^{(t)}\right)$ is almost orthogonal. This is the most delicate part of the argument. To prove this, we use induction on $m$ to show that $\left(\mathbf{x}_{1}^{(t)}, \ldots, \widehat{\mathbf{x}_{k t}^{(t)}}, \ldots, \mathbf{x}_{m}^{(t)}\right)$ is almost orthogonal for $m=\ell_{t}, \ldots, n$. When $m=\ell_{t}$, this follows from our construction, thanks to the last assertion of Lemma 5.1, because $\left(\mathbf{x}_{1}^{(t-1)}, \ldots, \widehat{\mathbf{x}_{k_{t-1}}^{(t-1)}}, \ldots, \mathbf{x}_{\ell_{t}}^{(t-1)}\right)$ is almost orthogonal. If $\ell_{t}=n$, we are done. Otherwise, fix an integer $m$ with $\ell_{t} \leq m<n$ and assume that our claim is true for that value of $m$. Since $\ell_{0}=n>m$, there exists an index $r$ with $0 \leq r<t$ such that

$$
\ell_{r}>m \text { and } \ell_{r+1}, \ldots, \ell_{t} \leq m \text {. }
$$

In particular, we have $k_{r}, \ldots, k_{t} \leq m$. Thus $\left(\mathbf{x}_{1}^{(i)}, \ldots, \widehat{\mathbf{x}_{k_{i}}^{(i)}}, \ldots, \mathbf{x}_{m}^{(i)}\right)$ is almost orthogonal for $i=r, \ldots, t$, while

$$
\left(\mathbf{x}_{1}^{(i)}, \ldots, \widehat{\mathbf{x}_{\ell_{i}}^{(i)}}, \ldots, \mathbf{x}_{m}^{(i)}\right)=\left(\mathbf{x}_{1}^{(i-1)}, \ldots, \widehat{\mathbf{x}_{k_{i-1}}^{(i-1)}}, \ldots, \mathbf{x}_{m}^{(i-1)}\right)
$$

is also almost orthogonal for $i=r+1, \ldots, t$. Define

$$
U^{(i)}=\left\langle\mathbf{x}_{1}^{(i)}, \ldots, \mathbf{x}_{m}^{(i)}\right\rangle_{\mathbb{R}} \quad \text { and } \quad V^{(i)}=\left\langle\mathbf{x}_{1}^{(i)}, \ldots, \widehat{\mathbf{x}_{k_{i}}^{(i)}}, \ldots, \mathbf{x}_{m}^{(i)}\right\rangle_{\mathbb{R}} \quad(r \leq i \leq t) .
$$

As $V^{(i-1)}=\left\langle\mathbf{x}_{1}^{(i)}, \ldots, \widehat{\mathbf{x}_{\ell_{i}}^{(i)}}, \ldots, \mathbf{x}_{m}^{(i)}\right\rangle_{\mathbb{R}}$ for $i=r+1, \ldots, t$, Lemma 4.7 gives

$$
\operatorname{dist}\left(V^{(i-1)}, V^{(i)}\right) \leq e^{4} \frac{H\left(U^{(i)}\right)}{\left\|\mathbf{x}_{1}^{(i)}\right\| \cdots\left\|\mathbf{x}_{m}^{(i)}\right\|} \quad(r+1 \leq i \leq t)
$$

By 5), we also have $U^{(i-1)}=V^{(i-1)}+\left\langle\mathbf{x}_{k_{i-1}}^{(i-1)}\right\rangle_{\mathbb{R}}=V^{(i-1)}+\left\langle\mathbf{x}_{\ell_{i}}^{(i)}\right\rangle_{\mathbb{R}}=U^{(i)}$ for $i=r+1, \ldots, t$. Thus $U^{(r)}=U^{(r+1)}=\cdots=U^{(t)}$ and therefore, using Lemma 4.3 , we obtain

$$
\operatorname{dist}\left(V^{(r)}, V^{(t)}\right) \leq \sum_{i=r+1}^{t} \operatorname{dist}\left(V^{(i-1)}, V^{(i)}\right) \leq e^{4} \sum_{i=r+1}^{t} \frac{H\left(U^{(r)}\right)}{\left\|\mathbf{x}_{1}^{(i)}\right\| \cdots\left\|\mathbf{x}_{m}^{(i)}\right\|} .
$$

With the help of 6$), 3)$, (5.2) and (5.3), we also note that

$$
\frac{\left\|\mathbf{x}_{1}^{(i-1)}\right\| \cdots\left\|\mathbf{x}_{m}^{(i-1)}\right\|}{\left\|\mathbf{x}_{1}^{(i)}\right\| \cdots\left\|\mathbf{x}_{m}^{(i)}\right\|}=\frac{\left\|\mathbf{x}_{k_{i-1}}^{(i-1)}\right\|}{\left\|\mathbf{x}_{\ell_{i}}^{(i)}\right\|} \leq \frac{2 A_{k_{i-1}}^{(i-1)}}{A_{\ell_{i}}^{(i)}} \leq \frac{2 A_{\ell_{i}}^{(i-1)}}{A_{\ell_{i}}^{(i)}} \leq \frac{2}{C}
$$

for $i=r+1, \ldots, t$. This yields

$$
\operatorname{dist}\left(V^{(r)}, V^{(t)}\right) \leq 2 e^{4} \frac{H\left(U^{(r)}\right)}{\left\|\mathbf{x}_{1}^{(r+1)}\right\| \cdots\left\|\mathbf{x}_{m}^{(r+1)}\right\|} \leq 2 e^{4} \frac{\left\|\mathbf{x}_{1}^{(r)}\right\| \cdots\left\|\mathbf{x}_{m}^{(r)}\right\|}{\left\|\mathbf{x}_{1}^{(r+1)}\right\| \cdots\left\|\mathbf{x}_{m}^{(r+1)}\right\|} \leq \frac{4 e^{4}}{C} \leq \frac{1}{2^{n}} .
$$

According to 6) and (5.5), we also have $\mathbf{x}_{m+1}^{(r)}=\mathbf{x}_{m+1}^{(r+1)}=\cdots=\mathbf{x}_{m+1}^{(t)}$. Therefore, we conclude, by Lemma 4.3, that

$$
\operatorname{dist}\left(\mathbf{x}_{m+1}^{(t)}, V^{(t)}\right) \geq \operatorname{dist}\left(\mathbf{x}_{m+1}^{(t)}, V^{(r)}\right)-\operatorname{dist}\left(V^{(r)}, V^{(t)}\right) \geq \operatorname{dist}\left(\mathbf{x}_{m+1}^{(r)}, V^{(r)}\right)-\frac{1}{2^{n}}
$$


We recall at this point that $\ell_{r}>m$. If $r>0$ and $\ell_{r}>m+1$, then $\left(\mathbf{x}_{1}^{(r)}, \ldots, \mathbf{x}_{m+1}^{(r)}\right)$ is almost orthogonal because it is a subsequence of

$$
\left(\mathbf{x}_{1}^{(r)}, \ldots, \widehat{\mathbf{x}_{\ell_{r}}^{(r)}}, \ldots, \mathbf{x}_{n}^{(r)}\right)=\left(\mathbf{x}_{1}^{(r-1)}, \ldots, \widehat{\mathbf{x}_{k_{r-1}}^{(r-1)}}, \ldots, \mathbf{x}_{n}^{(r-1)}\right)
$$

which is almost orthogonal. The same is true if $r=0$ and $n=\ell_{0}>m+1$ because, in that case, it is a subsequence of $\left(\mathbf{x}_{1}^{(0)}, \ldots, \mathbf{x}_{n-1}^{(0)}\right)$ which, by condition 1$)$, is almost orthogonal. So, if $\ell_{r}>m+1$, we obtain

$$
\operatorname{dist}\left(\mathbf{x}_{m+1}^{(r)}, V^{(r)}\right) \geq \operatorname{dist}\left(\mathbf{x}_{m+1}^{(r)}, U^{(r)}\right)=\operatorname{dist}\left(\mathbf{x}_{m+1}^{(r)},\left\langle\mathbf{x}_{1}^{(r)}, \ldots, \mathbf{x}_{m}^{(r)}\right\rangle_{\mathbb{R}}\right) \geq 1-\frac{1}{2^{m}}
$$

If $\ell_{r}=m+1$, the inequality $\operatorname{dist}\left(\mathbf{x}_{m+1}^{(r)}, V^{(r)}\right) \geq 1-1 / 2^{m}$ also holds as a direct consequence of 4) for $i=r$ because $k_{r} \leq m<\ell_{r}$ (in fact, this is precisely the reason why we need this condition 4)). So, in all cases, we conclude that

$$
\operatorname{dist}\left(\mathbf{x}_{m+1}^{(t)}, V^{(t)}\right) \geq \operatorname{dist}\left(\mathbf{x}_{m+1}^{(r)}, V^{(r)}\right)-\frac{1}{2^{n}} \geq 1-\frac{1}{2^{m}}-\frac{1}{2^{n}} \geq 1-\frac{1}{2^{m-1}} .
$$

Thus $\left(\mathbf{x}_{1}^{(t)}, \ldots, \widehat{\mathbf{x}_{k_{t}}^{(t)}}, \ldots, \mathbf{x}_{m+1}^{(t)}\right)$ is almost orthogonal, and our claim follows by the induction principle.

Having proved the first part of the proposition, we know that $\left(\mathbf{x}_{1}^{(i)}, \ldots, \widehat{\mathbf{x}_{k_{i}}^{(i)}}, \ldots, \mathbf{x}_{n}^{(i)}\right)$ is almost orthogonal for each $i$ with $0 \leq i<s$. Define

$$
V^{(i)}=\left\langle\mathbf{x}_{1}^{(i)}, \ldots, \widehat{\mathbf{x}_{k_{i}}^{(i)}}, \ldots, \mathbf{x}_{n}^{(i)}\right\rangle_{\mathbb{R}} \quad(0 \leq i<s) .
$$

When $i \geq 1$, the condition 6) gives $V^{(i-1)}=\left\langle\mathbf{x}_{1}^{(i)}, \ldots, \widehat{\mathbf{x}_{\ell_{i}}^{(i)}}, \ldots, \mathbf{x}_{n}^{(i)}\right\rangle_{\mathbb{R}}$ and so $V^{(i-1)}+V^{(i)}=$ $\left\langle\mathbf{x}_{1}^{(i)}, \ldots, \mathbf{x}_{n}^{(i)}\right\rangle_{\mathbb{R}}=\mathbb{R}^{n}$. Since $H\left(\mathbb{R}^{n}\right)=1$, we deduce from Lemmas 4.4 and 4.7 that

$$
\operatorname{dist}\left(\mathbf{u}_{i-1}, \mathbf{u}_{i}\right)=\operatorname{dist}\left(V^{(i-1)}, V^{(i)}\right) \leq \frac{e^{4}}{\left\|\mathbf{x}_{1}^{(i)}\right\| \cdots\left\|\mathbf{x}_{n}^{(i)}\right\|} .
$$

We also note that (5.6) holds with $m=n$ for any integer $i$ with $1 \leq i<s$. So, for integers $r, t$ with $0 \leq r<t<s$, we conclude that

$$
\operatorname{dist}\left(\mathbf{u}_{r}, \mathbf{u}_{t}\right) \leq \sum_{i=r+1}^{t} \operatorname{dist}\left(\mathbf{u}_{i-1}, \mathbf{u}_{i}\right) \leq \sum_{i=r+1}^{t} \frac{e^{4}}{\left\|\mathbf{x}_{1}^{(i)}\right\| \cdots\left\|\mathbf{x}_{n}^{(i)}\right\|} \leq \frac{2 e^{4}}{\left\|\mathbf{x}_{1}^{(r+1)}\right\| \cdots\left\|\mathbf{x}_{n}^{(r+1)}\right\|}
$$

The next result completes the above proposition by constructing a unit vector $\mathbf{u}$ and by estimating the distance function of the points $\mathbf{x}_{1}^{(i)}, \ldots, \mathbf{x}_{n}^{(i)}$ with respect to the convex body $\mathcal{C}_{\mathbf{u}}(Q)$ in appropriate ranges for $Q$.

Proposition 5.4. Let the notation be as in the previous proposition. Define

$$
Q_{i}=A_{1}^{(i)} \cdots A_{n}^{(i)} \quad(0 \leq i<s)
$$

and set $Q_{s}=\infty$ if $s \neq \infty$. Then there exists a unit vector $\mathbf{u} \in \mathbb{R}^{n}$ with the property that, for each $i$ with $0 \leq i<s$ and each $Q \in\left[Q_{i}, Q_{i+1}\right)$, we have

1) $A_{j}^{(i)} \leq \lambda\left(\mathbf{x}_{j}^{(i)}, \mathcal{C}_{\mathbf{u}}(Q)\right) \leq 8 e^{4} A_{j}^{(i)} \quad$ for any $j \in\{1, \ldots, n\}$ with $j \neq k_{i}$, 
2) $\frac{A_{k_{i}}^{(i)} Q}{2^{n} Q_{i}} \leq \lambda\left(\mathbf{x}_{k_{i}}^{(i)}, \mathcal{C}_{\mathbf{u}}(Q)\right) \leq \frac{8 A_{k_{i}}^{(i)} Q}{Q_{i}}$

Proof. We will use freely the inequalities $A_{j}^{(i)} \leq\left\|\mathbf{x}_{j}^{(i)}\right\| \leq 2 A_{j}^{(i)}(1 \leq j \leq n, 0 \leq i<s)$ coming from the condition 3) of Proposition [5.3, as well as the inequalities (5.2) to (5.4) involving the numbers $A_{j}^{(i)}$. The construction of the point $\mathbf{u}$ is based on the second assertion of Proposition 5.3. When $s=\infty$, this statement implies that the image of the sequence $\left(\mathbf{u}_{i}\right)_{i \geq 1}$ in $\mathbb{P}^{n-1}(\mathbb{R})$ converges to the class of a unit vector $\mathbf{u} \in \mathbb{R}^{n}$ such that

$$
\operatorname{dist}\left(\mathbf{u}_{i}, \mathbf{u}\right) \leq \frac{2 e^{4}}{\left\|\mathbf{x}_{1}^{(i+1)}\right\| \cdots\left\|\mathbf{x}_{n}^{(i+1)}\right\|} \quad(0 \leq i<s)
$$

When $s \neq \infty$, these inequalities remain true for the choice of $\mathbf{u}=\mathbf{u}_{s-1}$, provided that we interpret the right end side as 0 when $i=s-1$. We now replace each $\mathbf{u}_{i}$ by $\pm \mathbf{u}_{i}$ so that $\mathbf{u}_{i} \cdot \mathbf{u} \geq 0$. Then, we obtain

$$
\left\|\mathbf{u}_{i}-\mathbf{u}\right\| \leq 2 \operatorname{dist}\left(\mathbf{u}_{i}, \mathbf{u}\right) \leq \frac{4 e^{4}}{Q_{i+1}} \quad(0 \leq i<s) .
$$

For the rest of the argument, we fix an integer $i$ with $0 \leq i<s$ and a number $Q \in\left[Q_{i}, Q_{i+1}\right)$. Our goal is to estimate

$$
\lambda\left(\mathbf{x}_{j}^{(i)}, \mathcal{C}_{\mathbf{u}}(Q)\right)=\max \left\{\left\|\mathbf{x}_{j}^{(i)}\right\|,\left|\mathbf{x}_{j}^{(i)} \cdot \mathbf{u}\right| Q\right\} \quad(1 \leq j \leq n) .
$$

For each $j \in\{1, \ldots, n\}$, we have

$$
\left|\mathbf{x}_{j}^{(i)} \cdot\left(\mathbf{u}-\mathbf{u}_{i}\right)\right| \leq\left\|\mathbf{x}_{j}^{(i)}\right\|\left\|\mathbf{u}-\mathbf{u}_{i}\right\| \leq \frac{8 e^{4} A_{j}^{(i)}}{Q_{i+1}} .
$$

When $j \neq k_{i}$, the vector $\mathbf{u}_{i}$ is orthogonal to $\mathbf{x}_{j}^{(i)}$. Then, since $Q<Q_{i+1}$, we obtain

$$
\left|\mathbf{x}_{j}^{(i)} \cdot \mathbf{u}\right| Q \leq 8 e^{4} A_{j}^{(i)}
$$

and the inequalities 1) follow from (5.7).

Since $\left(\mathbf{x}_{1}^{(i)}, \ldots, \mathbf{x}_{n}^{(i)}\right)$ is a basis of $\mathbb{Z}^{n}$ and since its subsequence obtained by deleting $\mathbf{x}_{k_{i}}^{(i)}$ is almost orthogonal (and orthogonal to $\mathbf{u}_{i}$ ), Lemmas 4.1 and 4.6 give

$$
\frac{\left\|\mathbf{x}_{k_{i}}^{(i)}\right\|}{\left\|\mathbf{x}_{1}^{(i)}\right\| \cdots\left\|\mathbf{x}_{n}^{(i)}\right\|} \leq\left|\mathbf{x}_{k_{i}}^{(i)} \cdot \mathbf{u}_{i}\right|=\frac{1}{H\left(\left\langle\mathbf{x}_{1}^{(i)}, \ldots, \widehat{\mathbf{x}_{k_{i}}^{(i)}}, \ldots, \mathbf{x}_{n}^{(i)}\right\rangle_{\mathbb{R}}\right)} \leq \frac{e^{2}\left\|\mathbf{x}_{k_{i}}^{(i)}\right\|}{\left\|\mathbf{x}_{1}^{(i)}\right\| \cdots\left\|\mathbf{x}_{n}^{(i)}\right\|}
$$

and therefore

$$
\frac{A_{k_{i}}^{(i)}}{2^{n-1} Q_{i}} \leq\left|\mathbf{x}_{k_{i}}^{(i)} \cdot \mathbf{u}_{i}\right| \leq \frac{e^{2} A_{k_{i}}^{(i)}}{Q_{i}}
$$

If $i+1<s$, we also note that

$$
\frac{Q_{i+1}}{Q_{i}}=\frac{A_{\ell_{i+1}}^{(i+1)}}{A_{k_{i}}^{(i)}} \geq \frac{A_{\ell_{i+1}}^{(i+1)}}{A_{\ell_{i+1}}^{(i)}} \geq C=2^{n+3} e^{4},
$$


and so (5.8) yields

$$
\left|\mathbf{x}_{k_{i}}^{(i)} \cdot \mathbf{u}-\mathbf{x}_{k_{i}}^{(i)} \cdot \mathbf{u}_{i}\right|=\left|\mathbf{x}_{k_{i}}^{(i)} \cdot\left(\mathbf{u}-\mathbf{u}_{i}\right)\right| \leq \frac{A_{k_{i}}^{(i)}}{2^{n} Q_{i}} .
$$

This inequality also holds if $i+1=s$ because in that case $\mathbf{u}=\mathbf{u}_{i}$. So, using (5.9), we deduce that

$$
\frac{A_{k_{i}}^{(i)}}{2^{n} Q_{i}} \leq\left|\mathbf{x}_{k_{i}}^{(i)} \cdot \mathbf{u}\right| \leq \frac{8 A_{k_{i}}^{(i)}}{Q_{i}}
$$

The estimates 2) then follow from (5.7) with $j=k_{i}$ because $Q \geq Q_{i}$.

We conclude this section with the following result which establishes, in quantitative form, the second assertion of Theorem 1.3 for rigid $n$-systems with sufficiently large mesh.

Theorem 5.5. Let $\delta \geq 4+(n+3) \log 2$ and let $\mathbf{P}:\left[q_{0}, \infty\right) \rightarrow \mathbb{R}^{n}$ be a rigid $n$-system with mesh $\delta$. Then, there exists a unit vector $\mathbf{u} \in \mathbb{R}^{n}$ such that

$$
\sup _{q \geq q_{0}}\left\|\mathbf{P}(q)-\mathbf{L}_{\mathbf{u}}(q)\right\|_{\infty} \leq n \log \left(8 e^{4} n\right) .
$$

Proof. Consider the sequence of points $\left(\mathbf{a}^{(i)}\right)_{0 \leq i<s}$ and the sequences of integers $\left(k_{i}\right)_{0 \leq i<s}$ and $\left(\ell_{i}\right)_{0 \leq i<s}$ which form the canvas attached to $\mathbf{P}$ as in Definitions 1.1 and 1.2. For each integer $i$ with $0 \leq i<s$, we write $\mathbf{a}^{(i)}=\left(a_{1}^{(i)}, \ldots, a_{n}^{(i)}\right)$ and set

$$
A_{j}^{(i)}:=\exp \left(a_{j}^{(i)}\right) \quad(1 \leq j \leq n)
$$

Then, the conditions (C1)-(C3) of Definition 1.1 yield (5.1)-(5.4) and thus Propositions 5.3 and 5.4 apply. Consider the bases $\left(\mathbf{x}_{1}^{(i)}, \ldots, \mathbf{x}_{n}^{(i)}\right)$ of $\mathbb{Z}^{n}$ provided by the first proposition for $0 \leq i<s$ and the unit vector $\mathbf{u}$ of $\mathbb{R}^{n}$ provided by the second one. We claim that this unit vector has the required property. To show this, we first note that, in agreement with Definition 1.2 and Proposition 5.4, we have

$$
q_{i}=a_{1}^{(i)}+\cdots+a_{n}^{(i)}=\log Q_{i} \quad(0 \leq i<s)
$$

and $q_{s}=\log Q_{s}=\infty$ if $s \neq \infty$. Then, we fix an arbitrary integer $i$ with $0 \leq i<s$ and a real number $q \in\left[q_{i}, q_{i+1}\right)$. We also set $Q=e^{q}$, so that $Q \in\left[Q_{i}, Q_{i+1}\right)$. Since $\left(\mathbf{x}_{1}^{(i)}, \ldots, \mathbf{x}_{n}^{(i)}\right)$ is a basis of $\mathbb{Z}^{n}$, the successive minima of $\mathcal{C}_{\mathbf{u}}(Q)$ are bounded above by a permutation of the numbers $\lambda\left(\mathbf{x}_{j}^{(i)}, \mathcal{C}_{\mathbf{u}}(Q)\right)(1 \leq j \leq n)$, and so, in view of Proposition 5.4, they are bounded above by a permutation of the $n$ numbers $8 A_{k_{i}}^{(i)} Q / Q_{i}$ and $8 e^{4} A_{j}^{(i)}\left(1 \leq j \leq n, j \neq k_{i}\right)$. Taking logarithms, we deduce that the numbers $L_{\mathbf{u}, j}(q):=\log \lambda_{j}\left(\mathcal{C}_{\mathbf{u}}\left(e^{q}\right)\right)(1 \leq j \leq n)$ are bounded above by a permutation of $a_{k_{i}}^{(i)}+q-q_{i}+c$ and $a_{j}^{(i)}+c\left(1 \leq j \leq n, j \neq k_{i}\right)$, where $c=\log \left(8 e^{4}\right)$. In view of the formula for $\mathbf{P}(q)$ in Definition 1.2 , this means that

$$
L_{\mathbf{u}, j}(q) \leq P_{j}(q)+c \quad(1 \leq j \leq n) .
$$

where $P_{j}$ denotes the $j$-th component of $\mathbf{P}$. By (2.2) and (2.7), we also have

$$
q-n \log (n) \leq \sum_{j=1}^{n} L_{\mathbf{u}, j}(q) \quad \text { and } \quad \sum_{j=1}^{n}\left(P_{j}(q)+c\right)=q+n c .
$$


We conclude that $0 \leq\left(P_{j}(q)+c\right)-L_{\mathbf{u}, j}(q) \leq n(\log (n)+c)$ for each $j=1, \ldots, n$ and so

$$
\left\|\mathbf{L}_{\mathbf{u}}(q)-\mathbf{P}(q)\right\|_{\infty} \leq n(\log (n)+c)=n \log \left(8 e^{4} n\right) .
$$

\section{REDUCED SYSTEMS}

In this section and the next one, we now turn to the problem of approximating a given $(n, \gamma)$-system on $[0, \infty)$ by rigid $n$-systems of sufficiently large mesh, for $n \geq 2$. In the current section, we simply narrow the class of $(n, \gamma)$-systems that we need to consider.

Definition 6.1. Let $q_{0} \geq 0$. An $(n, \gamma)$-reduced system on $\left[q_{0}, \infty\right)$ is an $(n, \gamma)$-system $\mathbf{P}=$ $\left(P_{1}, \ldots, P_{n}\right):\left[q_{0}, \infty\right) \rightarrow \mathbb{R}^{n}$ with the property that, for any $j=1, \ldots, n-1$, any $a \geq q_{0}$ and any $b \geq a+n \gamma$ such that $P_{1}+\cdots+P_{j}$ is constant on $[a, b]$, the functions $P_{1}, \ldots, P_{j}$ are constant on $[a, b-n \gamma]$.

For example, any $(n, 0)$-system is already an $(n, 0)$-reduced system. The goal of this section is to prove the following result.

Proposition 6.2. Let $\mathbf{P}:[0, \infty) \rightarrow \mathbb{R}^{n}$ be an $(n, \gamma)$-system on $[0, \infty)$. There exists an $(n, 2 n \gamma)$-reduced system $\tilde{\mathbf{P}}:[0, \infty) \rightarrow \mathbb{R}^{n}$ such that $\|\mathbf{P}-\tilde{\mathbf{P}}\|_{\infty} \leq n \gamma$.

The proof goes through several steps, based on the following observation whose proof is left to the reader.

Lemma 6.3. Let $a, b \in \mathbb{R}$ with $a<b$, and let $M:[a, b] \rightarrow \mathbb{R}$ be a continuous piecewise linear function with slopes 0 and 1 . Then the map $\tilde{M}:[a, b] \rightarrow \mathbb{R}$ given by

$$
\tilde{M}(q)=\max \{M(a), M(b)+q-b\} \quad \text { for any } q \in[a, b]
$$

is continuous and piecewise linear with slopes 0 and 1 . Moreover, it satisfies

$$
M(a)=\tilde{M}(a) \leq \tilde{M}(q) \leq M(q) \leq M(b)=\tilde{M}(b)
$$

for each $q \in[a, b]$.

Write $\mathbf{P}=\left(P_{1}, \ldots, P_{n}\right)$ and set $M_{j}=P_{1}+\cdots+P_{j}$ for each $j=0, \ldots, n$. We define recursively a sequence of continuous piecewise linear functions $\tilde{M}_{n}, \ldots, \tilde{M}_{1}$ from $[0, \infty)$ to $\mathbb{R}$ with slopes 0 and 1 in the following way. We first set $\tilde{M}_{n}=M_{n}$ and $\tilde{M}_{n-1}=M_{n-1}$. Then, assuming that $\tilde{M}_{j}$ has been constructed for some index $j$ with $2 \leq j \leq n-1$, we form the set $\mathcal{E}_{j}$ of all maximal sub-intervals of $[0, \infty)$ with non-empty interior on which $\tilde{M}_{j}$ is constant. The end-points of these intervals are the points of $(0, \infty)$ where $\tilde{M}_{j}$ is not differentiable, and possibly the point 0 . So, they form a discrete subset of $[0, \infty)$. We define $\tilde{M}_{j-1}:[0, \infty) \rightarrow \mathbb{R}$ by

$$
\tilde{M}_{j-1}(q)= \begin{cases}\max \left\{M_{j-1}(a), M_{j-1}(b)+q-b\right\} & \text { if } q \in[a, b] \in \mathcal{E}_{j}, \\ M_{j-1}(a) & \text { if } q \in[a, \infty) \in \mathcal{E}_{j}, \\ M_{j-1}(q) & \text { if } q \notin I \text { for any } I \in \mathcal{E}_{j},\end{cases}
$$

Lemma 6.3 applied to the restriction of $M_{j-1}$ to any bounded interval $[a, b] \in \mathcal{E}_{j}$ shows that $\tilde{M}_{j-1}$ and $M_{j-1}$ agree at the end-points of such an interval. Moreover $\tilde{M}_{j-1}$ and $M_{j-1}$ agree at the point $a$ if $[a, \infty) \in \mathcal{E}_{j}$. Therefore, $\tilde{M}_{j-1}$ is continuous. Clearly it is piecewise linear with slopes 0 and 1 . Once $\tilde{M}_{n}, \ldots, \tilde{M}_{1}$ have been constructed, we set $\tilde{M}_{0}=0$. 
Lemma 6.4. Let $j \in\{1, \ldots, n-1\}$.

(i) For any $q \geq 0$, we have $\tilde{M}_{j}(q) \leq M_{j}(q) \leq \tilde{M}_{j}(q)+(n-j-1) \gamma$.

(ii) Suppose $j \geq 2$. Then, for any compact sub-interval $[c, d]$ of $[0, \infty)$ on which $\tilde{M}_{j}$ is constant, we have $0 \leq M_{j-1}(d)-\tilde{M}_{j-1}(c) \leq(n-j) \gamma$.

Proof. We proceed by descending induction on $j$. For $j=n-1$, the assertion (i) is clear because $\tilde{M}_{n-1}=M_{n-1}$. If $n=2$, there is nothing more to prove. Suppose that $n \geq 3$ and that (i) holds for some integer $j \in\{2, \ldots, n-1\}$. Let $[c, d]$ be a compact subinterval of $[0, \infty)$ on which $\tilde{M}_{j}$ is constant. If $[c, d]$ is not contained in any interval of $\mathcal{E}_{j}$, we must have $c=d$ and $\tilde{M}_{j-1}(c)=M_{j-1}(c)$, so (ii) holds. Otherwise $[c, d]$ is contained in an interval $I$ of $\mathcal{E}_{j}$ of the form $[a, b]$ or $[a, \infty)$. In the first case, Lemma 6.3 shows that $\tilde{M}_{j-1}(a)=M_{j-1}(a)$ and $\tilde{M}_{j-1}(c) \leq M_{j-1}(c)$. This is also true in the second case. Then, combining this with the fact that $M_{j-1}$ and $\tilde{M}_{j-1}$ are monotone increasing, we deduce that

$$
M_{j-1}(d)-\tilde{M}_{j-1}(c) \geq M_{j-1}(c)-\tilde{M}_{j-1}(c) \geq 0
$$

and

$$
\begin{aligned}
M_{j-1}(d)-\tilde{M}_{j-1}(c) & \leq M_{j-1}(d)-\tilde{M}_{j-1}(a) \\
& =M_{j-1}(d)-M_{j-1}(a) \\
& =\left(M_{j}(d)-M_{j}(a)\right)+\left(P_{j}(a)-P_{j}(d)\right) .
\end{aligned}
$$

Since $\mathbf{P}$ is an $(n, \gamma)$-system and since $a \leq d$ we also have $P_{j}(a)-P_{j}(d) \leq \gamma$. Moreover, the induction hypothesis gives $M_{j}(d) \leq \tilde{M}_{j}(d)+(n-j-1) \gamma$. As $\tilde{M}_{j}$ is constant on $I \supseteq[a, d]$, we further have $\tilde{M}_{j}(d)=\tilde{M}_{j}(a)=M_{j}(a)$. This means that $M_{j}(d)-M_{j}(a) \leq(n-j-1) \gamma$. So, we conclude that

$$
0 \leq M_{j-1}(d)-\tilde{M}_{j-1}(c) \leq(n-j) \gamma
$$

and therefore (ii) holds for that value of $j$. Applying this result with $c=d=q$ for an arbitrary $q \geq 0$, we obtain $\tilde{M}_{j-1}(q) \leq M_{j-1}(q) \leq \tilde{M}_{j-1}(q)+(n-j) \gamma$. Thus (i) holds with $j$ replaced by $j-1$. This completes the induction step and proves the lemma.

Lemma 6.5. Let $j \in\{1, \ldots, n-1\}$. Suppose that $\tilde{M}_{j}$ is constant on some compact subinterval $[c, d]$ of $[0, \infty)$ with $d \geq c+n \gamma$. Then $\tilde{M}_{j-1}$ is constant on $[c, d-n \gamma]$.

Proof. If $j=1$, this is true because $\tilde{M}_{0}=0$. Suppose that $j \geq 2$. Then, $[c, d]$ is contained in some interval of $\mathcal{E}_{j}$. If this interval is of the form $[a, \infty)$, then $\tilde{M}_{j-1}$ is constant on $[a, \infty)$ and so it is constant on $[c, d]$. Otherwise this interval is of the form $[a, b]$ and, on this interval, $\tilde{M}_{j-1}$ has slope 0 and then slope 1 . If $\tilde{M}_{j-1}$ is not constant on $[c, d-n \gamma]$, this means that it has slope 1 on $[d-n \gamma, b]$ and so we obtain

$$
n \gamma=\tilde{M}_{j-1}(d)-\tilde{M}_{j-1}(d-n \gamma) \leq \tilde{M}_{j-1}(d)-\tilde{M}_{j-1}(c) .
$$

This is impossible because Lemma 6.4 gives

$$
\tilde{M}_{j-1}(d)-\tilde{M}_{j-1}(c) \leq M_{j-1}(d)-\tilde{M}_{j-1}(c) \leq(n-j) \gamma .
$$

For each $j=1, \ldots, n$, we define $\tilde{P}_{j}=\tilde{M}_{j}-\tilde{M}_{j-1}$ so that

$$
\tilde{M}_{j}=\tilde{P}_{1}+\cdots+\tilde{P}_{j} \quad \text { for } j=1, \ldots, n .
$$


We claim that the resulting map $\tilde{\mathbf{P}}=\left(\tilde{P}_{1}, \ldots, \tilde{P}_{n}\right):[0, \infty) \rightarrow \mathbb{R}^{n}$ satisfies all the conditions of Proposition 6.2. It clearly satisfies the conditions (S3) and (S4) of Definition 2.8. The next two lemmas show that it satisfies all the other requirements.

Lemma 6.6. We have

(i) $\left|P_{j}(q)-\tilde{P}_{j}(q)\right| \leq(n-1) \gamma \quad(1 \leq j \leq n, 0 \leq q)$,

(ii) $-n \gamma \leq \tilde{P}_{j}(q) \leq \tilde{P}_{j+1}(q)+2 n \gamma \quad(1 \leq j<n, 0 \leq q)$,

(iii) $\tilde{P}_{j}\left(q_{1}\right) \leq \tilde{P}_{j}\left(q_{2}\right)+2 n \gamma \quad\left(1 \leq j \leq n, 0 \leq q_{1} \leq q_{2}\right)$.

Moreover, if, for some $j \in\{1, \ldots, n-1\}$, the function $\tilde{M}_{j}$ is constant on some compact subinterval $[c, d]$ of $[0, \infty)$ with $d \geq c+n^{2} \gamma$, then $\tilde{P}_{1}, \ldots, \tilde{P}_{j}$ are constant on $\left[c, d-n^{2} \gamma\right]$.

Proof. Let $q \geq 0$. Lemma 6.4(i) shows that $0 \leq M_{j}(q)-\tilde{M}_{j}(q) \leq(n-1) \gamma$ for $j=1, \ldots, n-1$. This is also true for $j=0$ and $j=n$ because in those cases we have $M_{j}=\tilde{M}_{j}$. Then Part (i) follows from the equalities

$$
P_{j}(q)-\tilde{P}_{j}(q)=\left(M_{j}(q)-\tilde{M}_{j}(q)\right)-\left(M_{j-1}(q)-\tilde{M}_{j-1}(q)\right) \quad(1 \leq j \leq n) .
$$

Let $j \in\{1, \ldots, n\}$. If $j<n$, the inequality $-\gamma \leq P_{j}(q) \leq P_{j+1}(q)+\gamma$ combined with the estimates of Part (i) yields

$$
\begin{aligned}
& \tilde{P}_{j}(q) \geq P_{j}(q)-(n-1) \gamma \geq-n \gamma \\
& \tilde{P}_{j}(q) \leq P_{j}(q)+(n-1) \gamma \leq P_{j+1}(q)+n \gamma \leq \tilde{P}_{j+1}(q)+(2 n-1) \gamma .
\end{aligned}
$$

Similarly, if $0 \leq q_{1} \leq q_{2}$, the inequality $P_{j}\left(q_{1}\right) \leq P_{j}\left(q_{2}\right)+\gamma$ yields

$$
\tilde{P}_{j}\left(q_{1}\right) \leq P_{j}\left(q_{1}\right)+(n-1) \gamma \leq P_{j}\left(q_{2}\right)+n \gamma \leq \tilde{P}_{j}\left(q_{2}\right)+(2 n-1) \gamma .
$$

This proves (ii) and (iii). Finally, if $\tilde{M}_{j}$ is constant on $[c, d]$ for some $j \in\{1, \ldots, n-1\}$ and some compact subinterval $[c, d]$ of $[0, \infty)$ of length at least $n^{2} \gamma$, then Lemma 6.5 shows that $\tilde{M}_{j}, \ldots, \tilde{M}_{1}$ are constant on $[c, d-n j \gamma]$ and so $\tilde{P}_{1}, \ldots, \tilde{P}_{j}$ are also constant on the latter interval.

Lemma 6.7. Let $j \in\{2, \ldots, n\}$. Suppose that $\tilde{M}_{j-1}$ changes slope from 1 to 0 at a point $q>0$. Then we have $\tilde{P}_{j}(q) \leq \tilde{P}_{j-1}(q)+2 n \gamma$.

Proof. Suppose first that $\tilde{M}_{j-1}$ coincides with $M_{j-1}$ on some open neighborhood of $q$. Then, as $M_{j-1}$ changes slope from 1 to 0 at $q$, we have $P_{j}(q) \leq P_{j-1}(q)+\gamma$ and the conclusion follows from Lemma 6.6 (i).

Suppose now that such a neighborhood does not exist. Then $q$ belongs to an interval $I$ of $\mathcal{E}_{j}$ and, since $\tilde{M}_{j-1}$ changes slope at most once on $I$, going from slope 0 to slope 1 , the point $q$ lies on the boundary of $I$. We claim that there is an interior point $p$ of $I$ at which $M_{j-1}$ changes slope from 1 to 0 . If $I=[a, b]$ is a compact interval, this is clear because otherwise $M_{j-1}$ would agree with $\tilde{M}_{j-1}$ on the whole interval $[a, b]$ and therefore would agree on an open interval containing $[a, b]$. If instead $I=[a, \infty)$ is unbounded, then $q=a$ and, since $M_{j-1}$ is constant on $[a, \infty)$, the function $M_{j-1}$, by differing locally from $\tilde{M}_{j-1}$, should have slope 1 in a right neighborhood $[a, a+\epsilon)$ of $a$, for some $\epsilon>0$. However, $M_{j-1}$ cannot have constant slope 1 on $[a, \infty)$ because $\tilde{M}_{j-1}$ is constant on $[a, \infty)$ and, by Lemma 6.4 (i), 
the difference $M_{j-1}-\tilde{M}_{j-1}$ is bounded. Thus the claim also holds in that case as well. By definition of an $(n, \gamma)$-system, we have

$$
P_{j}(p) \leq P_{j-1}(p)+\gamma .
$$

Put $c=\min \{p, q\}$ and $d=\max \{p, q\}$. Since $\tilde{M}_{j}$ is constant on $I \supseteq[c, d]$, we find, with the help of Lemma 6.4,

$$
\begin{aligned}
\tilde{P}_{j}(q)-P_{j}(p) & =\left(\tilde{M}_{j}(q)-M_{j}(p)\right)+\left(M_{j-1}(p)-\tilde{M}_{j-1}(q)\right) \\
& \leq\left(\tilde{M}_{j}(p)-M_{j}(p)\right)+\left(M_{j-1}(d)-\tilde{M}_{j-1}(c)\right) \leq(n-j) \gamma .
\end{aligned}
$$

Since $p \leq d$, we also have $P_{j-1}(p) \leq P_{j-1}(d)+\gamma$, and thus

$$
\begin{aligned}
P_{j-1}(p)-\tilde{P}_{j-1}(q) & \leq P_{j-1}(d)-\tilde{P}_{j-1}(q)+\gamma \\
& =\left(M_{j-1}(d)-\tilde{M}_{j-1}(q)\right)+\left(\tilde{M}_{j-2}(q)-M_{j-2}(d)\right)+\gamma \\
& \leq\left(M_{j-1}(d)-\tilde{M}_{j-1}(c)\right)+\left(\tilde{M}_{j-2}(d)-M_{j-2}(d)\right)+\gamma \\
& \leq(n-j+1) \gamma .
\end{aligned}
$$

Combining the three displayed estimates, we get $\tilde{P}_{j}(q)-\tilde{P}_{j-1}(q) \leq 2(n-j+1) \gamma \leq 2 n \gamma$.

\section{Approximation By RIGID n-Systems}

We shall now prove the following result.

Proposition 7.1. Let $\gamma, \delta \in \mathbb{R}$ with $0 \leq \gamma<\delta /\left(2 n^{2}\right)$ and let $\mathbf{P}:[0, \infty) \rightarrow \mathbb{R}^{n}$ be an $(n, \gamma)$ reduced system. Put $q_{0}=n(n+1) \delta / 2$. Then there exists a rigid $n$-system $\mathbf{R}:\left[q_{0}, \infty\right) \rightarrow \mathbb{R}^{n}$ of mesh $\delta$ such that $\|\mathbf{P}(q)-\mathbf{R}(q)\|_{\infty} \leq 3 n^{2} \delta$ for each $q \geq q_{0}$.

We first note that a change of variables reduces the proof to the case where $\delta=1$. Indeed, suppose for the moment that the proposition holds in that case and let us use the more suggestive terminology rigid integral $n$-system to denote a rigid $n$-system of mesh 1. Under the hypotheses of the proposition, we form the map $\tilde{\mathbf{P}}:[0, \infty) \rightarrow \mathbb{R}^{n}$ given by $\tilde{\mathbf{P}}(q)=\delta^{-1} \mathbf{P}(q \delta)$ for each $q \geq 0$. Then $\tilde{\mathbf{P}}$ is an $(n, \gamma / \delta)$-reduced system on $[0, \infty)$. As $\gamma / \delta<1 /\left(2 n^{2}\right)$, there exists a rigid integral $n$-system $\tilde{\mathbf{R}}:\left[q_{0}, \infty\right) \rightarrow \mathbb{R}^{n}$ with $q_{0}=n(n+1) / 2$ such that $\|\tilde{\mathbf{P}}(q)-\tilde{\mathbf{R}}(q)\|_{\infty} \leq 3 n^{2}$ for each $q \geq q_{0}$. The map $\mathbf{R}:\left[q_{0} \delta, \infty\right) \rightarrow \mathbb{R}^{n}$ given by $\mathbf{R}(q)=\delta \tilde{\mathbf{R}}(q / \delta)$ for each $q \geq q_{0} \delta$ is then a rigid $n$-system of mesh $\delta$ which satisfies $\|\mathbf{P}(q)-\mathbf{R}(q)\|_{\infty} \leq 3 n^{2} \delta$ for each $q \geq q_{0} \delta$ as requested.

So, from now on, we assume that $\delta=1$. We fix a real number $\gamma$ with $0 \leq \gamma<1 /\left(2 n^{2}\right)$ and an $(n, \gamma)$-reduced system $\mathbf{P}=\left(P_{1}, \ldots, P_{n}\right)$ on $[0, \infty)$. For each $j \in\{1, \ldots, n\}$, we define a new function $\bar{P}_{j}:[0, \infty) \rightarrow \mathbb{R}$ by putting

$$
\bar{P}_{j}(q)=\gamma+\sup \left\{P_{j}(t) ; 0 \leq t \leq q\right\} \quad(q \geq 0) .
$$

Then $\bar{P}_{1}, \ldots, \bar{P}_{n}$ are continuous piecewise linear functions with slopes 0 and 1 . The conditions (S1) and (S2) of Definition 2.8 respectively imply that, for each $q \geq 0$, they satisfy

$$
\begin{aligned}
0 & \leq \bar{P}_{j}(q) \leq \bar{P}_{j+1}(q)+\gamma & & (1 \leq j<n), \\
P_{j}(q)+\gamma & \leq \bar{P}_{j}(q) \leq P_{j}(q)+2 \gamma & & (1 \leq j \leq n) .
\end{aligned}
$$


Moreover, we note that $\bar{P}_{1}=\gamma+P_{1}$ and $\bar{P}_{n}=\gamma+P_{n}$ since $P_{1}$ and $P_{n}$ do not take negative slope.

We also define recursively a sequence of functions $E_{1}, \ldots, E_{n}$ from $[0, \infty)$ to $\mathbb{N}^{*}$ by putting, for each $q \geq 0$,

$$
\begin{aligned}
& E_{1}(q)=\left\lfloor\bar{P}_{1}(q)\right\rfloor+1, \\
& E_{j}(q)=\max \left\{E_{j-1}(q)+1,\left\lfloor\bar{P}_{j}(q)-2(j-1) \gamma\right\rfloor+1\right\} \text { for } j=2, \ldots, n,
\end{aligned}
$$

where $\lfloor x\rfloor$ stands for the integral part of a real number $x$. Then each $E_{j}$ is monotone increasing and right continuous. Moreover, the set of points of discontinuity of $E_{j}$ is discrete and, at such a point $q$, we have $E_{j}(q)=E_{j}\left(q^{-}\right)+1$, where $E_{j}\left(q^{-}\right)$is a shorthand for $\lim _{t \rightarrow q^{-}} E_{j}(t)$. These functions make up a map

$$
\mathbf{E}=\left(E_{1}, \ldots, E_{n}\right):[0, \infty) \rightarrow\left(\mathbb{N}^{*}\right)^{n}
$$

whose values form strictly increasing sequences of positive integers $0<E_{1}(q)<\cdots<E_{n}(q)$. Condition (S4) of Definition 2.8 together with (17.2) and the fact that each $\bar{P}_{j}$ has slopes 0 and 1 implies that

$$
\bar{P}_{j}(q) \leq q+\bar{P}_{j}(0) \leq q+\sum_{k=1}^{n}\left(P_{k}(0)+2 \gamma\right)=q+2 n \gamma<1 \quad(1 \leq j \leq n, 0 \leq q \leq 1 / 2),
$$

and so we obtain

$$
\mathbf{E}(q)=(1,2, \ldots, n) \quad(0 \leq q \leq 1 / 2) .
$$

The next lemma compares the functions $E_{j}$ and $\bar{P}_{j}$.

Lemma 7.2. For each $q \geq 0$, we have

(i) $\bar{P}_{j}(q)-2(j-1) \gamma<E_{j}(q) \leq \bar{P}_{j}(q)+j(1+\gamma)$ for $j=1, \ldots, n$,

(ii) $\|\mathbf{E}(q)-\mathbf{P}(q)\|_{\infty} \leq n+1$,

(iii) $\left|\left(E_{1}(q)+\cdots+E_{n}(q)\right)-q\right| \leq n(n+1)$.

Proof. Fix $q \geq 0$. For $j=1$, the inequality (i) is clear because $E_{1}(q)$ is the smallest integer which is greater than $\bar{P}_{1}(q)$. Suppose that this inequality holds for some integer $j$ with $1 \leq j<n$. We find

$$
\bar{P}_{j+1}(q)-2 j \gamma<\left\lfloor\bar{P}_{j+1}(q)-2 j \gamma\right\rfloor+1 \leq E_{j+1}(q)
$$

and, using the induction hypothesis together with (7.1), we obtain

$$
\begin{aligned}
E_{j+1}(q) & \leq \max \left\{E_{j}(q)+1, \bar{P}_{j+1}(q)-2 j \gamma+1\right\} \\
& \leq \max \left\{\bar{P}_{j}(q)+j(1+\gamma)+1, \bar{P}_{j+1}(q)+1\right\} \\
& \leq \bar{P}_{j+1}(q)+(j+1)(1+\gamma) .
\end{aligned}
$$

So, the inequality (i) is also satisfied with $j$ replaced by $j+1$. Consequently, it holds for $j=1, \ldots, n$. Using (7.2) and the fact that $\gamma \leq 1 /\left(2 n^{2}\right)$, this implies that

$$
\left|E_{j}(q)-P_{j}(q)\right| \leq \max \{2(j-1) \gamma, j(1+\gamma)+2 \gamma\} \leq n+1 \quad(1 \leq j \leq n),
$$

which proves (ii). Then (iii) follows because $P_{1}(q)+\cdots+P_{n}(q)=q$. 
Lemma 7.3. Let $(a, b)$ be an open sub-interval of $[0, \infty)$ of length $b-a \leq 1$. Each of the functions $E_{1}, \ldots, E_{n}$ admits at most one point of discontinuity on $(a, b)$.

Proof. Suppose on the contrary that, for some $j \in\{1, \ldots, n\}$, the function $E_{j}$ admits at least two points of discontinuity $q_{1}<q_{2}$ on $(a, b)$. Let $j$ be the minimal index with this property and choose $q_{1}, q_{2}$ so that $E_{j}$ is constant on $\left(q_{1}, q_{2}\right)$. Then, we have

$$
E_{j}\left(q_{1}^{-}\right)=H-1, \quad E_{j}\left(q_{1}\right)=E_{j}\left(q_{2}^{-}\right)=H \quad \text { and } \quad E_{j}\left(q_{2}\right)=H+1
$$

for some $H \in \mathbb{Z}$. Since $\bar{P}_{j}$ is continuous, we deduce from Lemma 7.2 that

$$
\bar{P}_{j}\left(q_{1}\right) \leq E_{j}\left(q_{1}^{-}\right)+2(j-1) \gamma=H-1+2(j-1) \gamma .
$$

As $\bar{P}_{j}$ has slope at most 1 and as $q_{2}-q_{1}<b-a \leq 1$, this gives

$$
\bar{P}_{j}\left(q_{2}\right)<\bar{P}_{j}\left(q_{1}\right)+1 \leq H+2(j-1) \gamma,
$$

thus $\left\lfloor\bar{P}_{j}\left(q_{2}\right)-2(j-1) \gamma\right\rfloor+1 \leq H$. Since $E_{j}\left(q_{2}\right)=H+1$, this implies that $j \geq 2$ and that $E_{j-1}$ is discontinuous at the point $q_{2}$, with $E_{j-1}\left(q_{2}\right)=H$. By virtue of the choice of $j$, this point $q_{2}$ must be the only point of discontinuity of $E_{j-1}$ on $(a, b)$. So, $E_{j-1}$ is constant on $\left(a, q_{2}\right)$, equal to $E_{j-1}\left(q_{2}^{-}\right)=H-1$. This means in particular that $E_{j-1}\left(q_{1}^{-}\right)=H-1=E_{j}\left(q_{1}^{-}\right)$, a contradiction.

Let $\Sigma$ denote the set of all points of discontinuity of $\mathbf{E}$ in $[0, \infty)$. Since, by (7.3), $\mathbf{E}$ is constant on $[0,1 / 2]$, the above lemma shows that $\Sigma$ is a discrete subset of $[1 / 2, \infty)$. It is also infinite because, by Lemma 7.2 (iii), the sum $E_{1}+\cdots+E_{n}$ is unbounded while it increases by at most $n$ at each point of discontinuity of $\mathbf{E}$. We now study

$$
\bar{\Sigma}:=\Sigma+[-n \gamma, n \gamma]=\{t \in \mathbb{R} ;|t-q| \leq n \gamma \text { for some } q \in \Sigma\} \subset[0, \infty) .
$$

Lemma 7.4. The set $\bar{\Sigma}$ has infinitely many connected components. Any such component $I$ is a closed interval of length at least $2 n \gamma$ and at most $2 n^{2} \gamma<1$, on which each of the functions $E_{1}, \ldots, E_{n}$ admits at most one point of discontinuity.

Proof. Since $\Sigma$ is a discrete subset of $\mathbb{R}$, the set $\bar{\Sigma}$ is a union of disjoint closed intervals of length at least $2 n \gamma$. Let $I$ be one of these, and let $[a, b]$ be a compact subinterval of $I$. The intersection of $\Sigma$ with $[a-n \gamma, b+n \gamma]$ consists of finitely many points $q_{1}<\cdots<q_{s}$. For these points, we have

$$
[a, b] \subseteq\left[q_{1}-n \gamma, q_{1}+n \gamma\right] \cup \cdots \cup\left[q_{s}-n \gamma, q_{s}+n \gamma\right]
$$

and $q_{i+1} \leq q_{i}+2 n \gamma$ for $i=1, \ldots, s-1$. If $s \geq n+1$, then one of the functions $E_{1}, \ldots, E_{n}$ admits at least two points of discontinuity among $q_{1}, \ldots, q_{n+1}$. By Lemma 7.3 , this is impossible because $\left[q_{1}, q_{n+1}\right]$ has length $q_{n+1}-q_{1} \leq 2 n^{2} \gamma<1$. Thus, we must have $s \leq n$, and the inclusion (7.4) yields $b-a \leq 2 n s \gamma \leq 2 n^{2} \gamma$. This shows that $I$ is bounded of length at most $2 n^{2} \gamma<1$ and so, again by Lemma 7.3, each of the functions $E_{1}, \ldots, E_{n}$ has at most one point of discontinuity in $I$. Finally, $\bar{\Sigma}$ consists of infinitely many disjoint such intervals because it contains $\Sigma$ which is infinite and discrete. 
In view of the above lemma, we can write

$$
\bar{\Sigma}=\left[c_{0}, d_{0}\right] \cup\left[c_{1}, d_{1}\right] \cup \cdots
$$

for an infinite sequence of real numbers $0 \leq c_{0}<d_{0}<c_{1}<d_{1}<\cdots$ with

$$
2 n \gamma \leq d_{i}-c_{i}<1 \quad(i \geq 0)
$$

For each $i \geq 0$, we denote by $\Omega^{(i)}$ the non-empty set of indices $j \in\{1, \ldots, n\}$ for which $E_{j}$ is not constant on $\left[c_{i}, d_{i}\right]$, and we define

$$
\begin{aligned}
k^{(i)} & :=\max \left\{j \in \Omega^{(i)} ; j=1 \text { or } E_{j-1}\left(d_{i}\right)<E_{j}\left(d_{i}\right)-1\right\}, \\
\ell^{(i)} & :=\min \left\{j \in \Omega^{(i)} ; j=n \text { or } E_{j+1}\left(c_{i}\right)>E_{j}\left(c_{i}\right)+1\right\} .
\end{aligned}
$$

Our next goal is to show that $\ell^{(i)} \geq k^{(i+1)}$ for each $i \geq 0$. This is the most delicate point. Once it is settled, the construction of the requested rigid integral $n$-system goes quickly as the reader could see by going directly to Lemma 7.8.

To prove the above inequality, we use freely the estimates (7.1) and (7.2). For each $j \in\{1, \ldots, n\}$, we set, as usual, $M_{j}=P_{1}+\cdots+P_{j}$. We also use the fact that, if $j<n$ and if $P_{j}+\gamma<P_{j+1}$ on a subinterval $[a, b]$ of $[0, \infty)$, then, by Condition (S5) of Definition 2.8, the function $M_{j}$ is concave up on $[a, b]$ : either its slope is constant on $[a, b]$, or it is 0 on $[a, c]$ and 1 on $[c, b]$ for some $c \in(a, b)$. We write $f^{\prime}\left(q^{-}\right)$to denote the left derivative of a function $f$ at a point $q$ and, for each $q \in \Sigma$, we denote by $\Omega(q)$ the non-empty set of indices $j \in\{1, \ldots, n\}$ such that $E_{j}$ is discontinuous at $q$. We first establish two lemmas whose proofs are illustrated on Figure 3.

Lemma 7.5. Let $q \in \Sigma$ and $k \in \Omega(q)$. Suppose that $k>1$ and that $E_{k-1}(q)<E_{k}(q)-1$. Then, we have $P_{k}^{\prime}\left(q^{-}\right)=1$ and $P_{k}(q)=E_{k}\left(q^{-}\right)+(2 k-3) \gamma$. Moreover, the function $M_{k-1}$ is constant on $[q-1, q]$.

Proof. Set $H=E_{k}\left(q^{-}\right)$so that $E_{k}(q)=H+1$ and $E_{k-1}(q) \leq H-1$. Since $E_{k-1}(q)+1<$ $E_{k}(q)$, we must have

$$
\left\lfloor\bar{P}_{k}(q)-2(k-1) \gamma\right\rfloor+1=E_{k}(q)=H+1,
$$

and so $\bar{P}_{k}(q) \geq H+2(k-1) \gamma$. Moreover, for each $t \in[0, q)$, Lemma 7.2 gives

$$
\bar{P}_{k}(t)<E_{k}(t)+2(k-1) \gamma \leq H+2(k-1) \gamma \leq \bar{P}_{k}(q) .
$$

This is incompatible with having either $P_{k}^{\prime}\left(q^{-}\right)=0$ or $P_{k}(q)+\gamma<\bar{P}_{k}(q)$. So, we must have $P_{k}^{\prime}\left(q^{-}\right)=1$ and, by letting $t$ tend to $q$ with $t<q$, we conclude that $P_{k}(q)=\bar{P}_{k}(q)-\gamma=$ $H+(2 k-3) \gamma$. This proves the first assertion of the lemma.

Since $E_{k-1}(q) \leq H-1$, Lemma $[7.2$ shows that, for any $t \in[0, q]$, we have

$$
P_{k-1}(t) \leq \bar{P}_{k-1}(q)<E_{k-1}(q)+2(k-2) \gamma \leq H-1+2(k-2) \gamma .
$$

As $P_{k}$ is continuous and piecewise linear with slope at most 1 , we conclude that, for any $t \in[q-1, q]$, we have

$$
P_{k}(t) \geq P_{k}(q)-1=H-1+(2 k-3) \gamma>P_{k-1}(t)+\gamma .
$$


Thus $M_{k-1}$ is concave up on $[q-1, q]$. However, the inequalities

$$
0 \leq M_{k-1}^{\prime}\left(q^{-}\right)=M_{k}^{\prime}\left(q^{-}\right)-P_{k}^{\prime}\left(q^{-}\right) \leq 1-P_{k}^{\prime}\left(q^{-}\right)=0
$$

show that $M_{k-1}^{\prime}\left(q^{-}\right)=0$ and therefore $M_{k-1}$ must be constant on $[q-1, q]$.
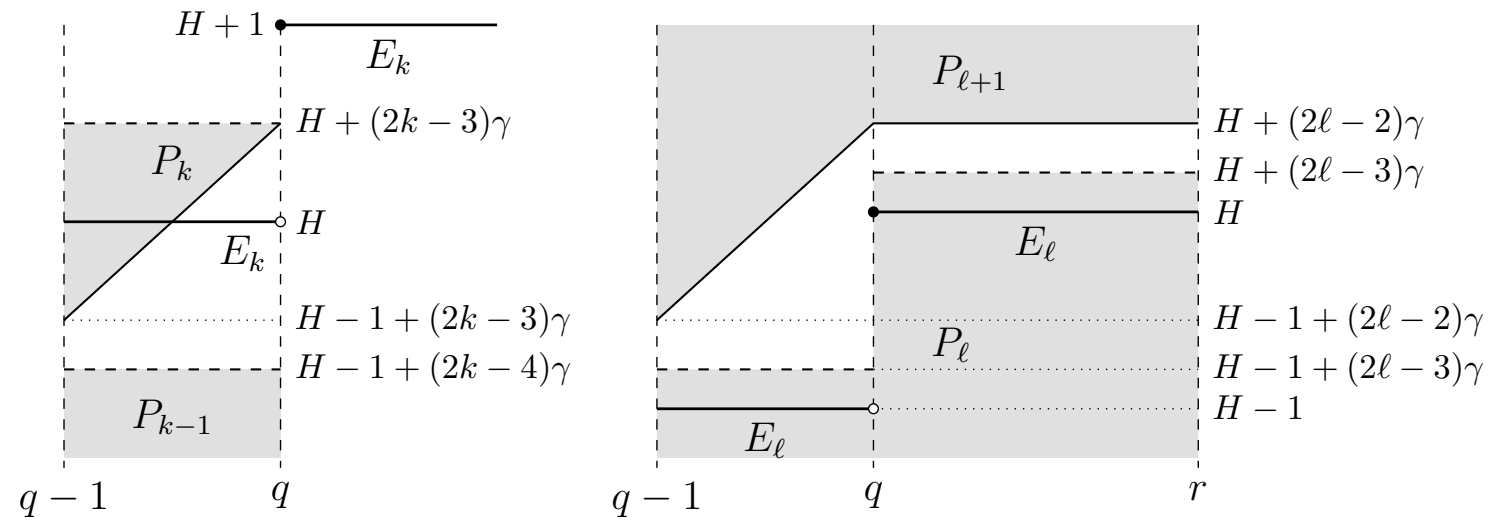

FiguRE 3. Illustrations for the proofs of Lemma 7.5 on the left, and of Lemma 7.6 on the right. The shaded regions contain the graphs of $P_{k-1}$ and $P_{k}$ on the left, and those of $P_{\ell}$ and $P_{\ell+1}$ on the right, showing the gap between them.

Lemma 7.6. Let $q \in \Sigma$ and $\ell \in \Omega(q)$. Suppose that $\ell<n$ and that $E_{\ell+1}\left(q^{-}\right)>E_{\ell}\left(q^{-}\right)+1$. Choose $r>q$ such that $E_{\ell}$ is constant on $[q, r)$. Then, $M_{\ell}$ is concave up on the interval $[q-1, r]$.

Proof. Set $H=E_{\ell}(q)$ so that $E_{\ell}\left(q^{-}\right)=H-1$ and $E_{\ell+1}\left(q^{-}\right) \geq H+1$. Choose $\epsilon>0$ such that $E_{\ell}$ and $E_{\ell+1}$ are constant on $[q-\epsilon, q)$. For each $t \in[q-\epsilon, q)$, we have $E_{\ell}(t)+1=H<E_{\ell+1}(t)$ and therefore

$$
H+1 \leq E_{\ell+1}(t)=\left\lfloor\bar{P}_{\ell+1}(t)-2 \ell \gamma\right\rfloor+1 \leq \bar{P}_{\ell+1}(t)-2 \ell \gamma+1 .
$$

Letting $t$ tend to $q$ and using the fact that $\bar{P}_{\ell+1}$ is continuous, we deduce that

$$
\bar{P}_{\ell+1}(q) \geq H+2 \ell \gamma \text {. }
$$

By Lemma 7.3, the function $E_{\ell}$ has no point of discontinuity on $(q-1, q)$. Since it is right continuous, it is therefore constant equal to $H-1$ on $[q-1, q)$. Then, for any $t \in[q-1, q)$, Lemma 7.2 yields

$$
P_{\ell}(t) \leq \bar{P}_{\ell}(t)-\gamma<H-1+(2 \ell-3) \gamma .
$$

Since $P_{\ell+1}$ has slope at most 1, we deduce from (17.6) and the preceding estimate that, for $t \in[q-1, q)$, we have

$$
P_{\ell+1}(t) \geq P_{\ell+1}(q)-1 \geq \bar{P}_{\ell+1}(q)-2 \gamma-1 \geq H-1+(2 \ell-2) \gamma>P_{\ell}(t)+\gamma .
$$

On the other hand, since $E_{\ell}$ is constant equal to $H$ on $[q, r)$, Lemma 7.2 combined with (7.6) shows that, for any $t \in[q, r)$, we have

$$
P_{\ell}(t)+\gamma \leq \bar{P}_{\ell}(t)<H+2(\ell-1) \gamma \leq \bar{P}_{\ell+1}(q)-2 \gamma \leq \bar{P}_{\ell+1}(t)-2 \gamma \leq P_{\ell+1}(t) .
$$


Thus $P_{\ell+1}(t)>P_{\ell}(t)+\gamma$ for any $t \in[q-1, r)$ and consequently, $M_{\ell}$ is concave up on $[q-1, r]$.

Our next lemma uses in a crucial way the hypothesis that $\mathbf{P}$ is reduced.

Lemma 7.7. We have $\ell^{(i)} \geq k^{(i+1)}$ for each $i \geq 0$.

Proof. Suppose on the contrary that $\ell^{(i)}<k^{(i+1)}$ for some $i \geq 0$. For simplicity, we write $\ell:=\ell^{(i)}$ and $k^{\prime}:=k^{(i+1)}$. Let $q \in\left[c_{i}+n \gamma, d_{i}-n \gamma\right]$ be the single point of discontinuity of $E_{\ell}$ on $\left[c_{i}, d_{i}\right]$ and let $q^{\prime} \in\left[c_{i+1}+n \gamma, d_{i+1}-n \gamma\right]$ be the one of $E_{k^{\prime}}$ on $\left[c_{i+1}, d_{i+1}\right]$. Since $\ell<k^{\prime}$, we have $k^{\prime}>1$ and $\ell<n$. The definition of $k^{\prime}=k^{(i+1)}$ in (7.5) yields

$$
E_{k^{\prime}-1}\left(q^{\prime}\right) \leq E_{k^{\prime}-1}\left(d_{i+1}\right)<E_{k^{\prime}}\left(d_{i+1}\right)-1=E_{k^{\prime}}\left(q^{\prime}\right)-1 .
$$

Thus Lemma 7.5 applies and shows that $M_{k^{\prime}-1}$ is constant on $\left[q^{\prime}-1, q^{\prime}\right]$. As $\mathbf{P}$ is $(n, \gamma)$ reduced, and as $q^{\prime}-n \gamma \geq c_{i+1}$, we deduce that $P_{1}, \ldots, P_{k^{\prime}-1}$ are constant on $\left[q^{\prime}-1, c_{i+1}\right]$. Note that this is an interval of positive length because $c_{i+1}>d_{i+1}-1 \geq q^{\prime}-1$. Since $\ell<k^{\prime}$, we conclude that $M_{\ell}=P_{1}+\cdots+P_{\ell}$ is also constant on $\left[q^{\prime}-1, c_{i+1}\right]$.

Similarly, we find that

$$
E_{\ell+1}\left(q^{-}\right) \geq E_{\ell+1}\left(c_{i}\right)>E_{\ell}\left(c_{i}\right)+1=E_{\ell}\left(q^{-}\right)+1 .
$$

Moreover, the function $E_{\ell}$ is constant on $\left[q, c_{i+1}\right]$ (because $\mathbf{E}$ is constant on $\left[d_{i}, c_{i+1}\right]$ ). Then, Lemma 7.6 shows that $M_{\ell}$ is concave up on $\left[q-1, c_{i+1}\right]$. As it is constant on $\left[q^{\prime}-1, c_{i+1}\right]$, it must therefore be constant on the whole interval $\left[q-1, c_{i+1}\right]$. Using again the fact that $\mathbf{P}$ is $(n, \gamma)$-reduced, this implies that $P_{\ell}$ is constant on $\left[q-1, c_{i+1}-n \gamma\right]$. This is a contradiction because $q$ is an interior point of the latter interval (since $q \leq d_{i}-n \gamma<c_{i+1}-n \gamma$ ) and, by hypothesis, $P_{\ell}$ is discontinuous at the point $q$.

The next two lemmas complete the proof of Proposition 7.1 when $\delta=1$.

Lemma 7.8. Define $t_{i}:=E_{1}\left(c_{i}\right)+\cdots+E_{n}\left(c_{i}\right)$ for each $i \geq 0$. Then, we have $t_{0}=n(n+1) / 2$ and there exists a rigid integer $n$-system $\mathbf{R}:\left[t_{0}, \infty\right) \rightarrow \Delta_{n}$ such that $\mathbf{R}\left(t_{i}\right)=\mathbf{E}\left(c_{i}\right)$ for each $i \geq 0$.

Proof. Since $\mathbf{E}$ is constant on $\left[0, c_{0}\right]$, we have $\mathbf{E}\left(c_{0}\right)=\mathbf{E}(0)=(1, \ldots, n)$ by (7.3) and thus $t_{0}=n(n+1) / 2$. For each $i \geq 0$, we partition $\Omega^{(i)}$ into maximal subsets of consecutive integers $\{k, \ldots, \ell\}$ such that $E_{k}\left(c_{i}\right), \ldots, E_{\ell}\left(c_{i}\right)$ are also consecutive integers and we order these subsets in a sequence $\Omega_{1}^{(i)}, \ldots, \Omega_{r_{i}}^{(i)}$ so that $\min \Omega_{r}^{(i)}>\max \Omega_{r+1}^{(i)}$ if $1 \leq r<r_{i}$. Then we group all of these into a single sequence

$$
\left(\Omega_{i}\right)_{i \geq 0}=\left(\Omega_{1}^{(0)}, \ldots, \Omega_{r_{0}}^{(0)}, \Omega_{1}^{(1)}, \ldots, \Omega_{r_{1}}^{(1)}, \ldots\right)
$$

and we define

$$
k_{i}=\min \Omega_{i}, \quad \ell_{i+1}=\max \Omega_{i} \quad(i \geq 0) .
$$

Clearly, we have $1 \leq k_{i} \leq \ell_{i+1} \leq n$ for each $i \geq 0$. By definition of $k^{(i)}$ and $\ell^{(i)}$ in (7.5), we also note that

$$
k^{(i)}=\min \Omega_{1}^{(i)} \quad \text { and } \quad \ell^{(i)}=\max \Omega_{r_{i}}^{(i)} \quad(i \geq 0) .
$$


Thus the inequality $\ell^{(i)} \geq k^{(i+1)}$ of Lemma 7.7 translates into $\max \Omega_{r_{i}}^{(i)} \geq \min \Omega_{1}^{(i+1)}$ for each $i \geq 0$. Since we also have $\max \Omega_{r}^{(i)}>\min \Omega_{r+1}^{(i)}$ when $1 \leq r<r_{i}$, we conclude that $\max \Omega_{i} \geq \min \Omega_{i+1}$ for each $i \geq 0$, and so $\ell_{i} \geq k_{i}$ for each $i \geq 1$. Upon setting $\ell_{0}=n$, the latter inequality extends to all $i \geq 0$.

For each $i \geq 0$ and each $r=1, \ldots, r_{i}$, we define an integer point $\mathbf{a}_{r}^{(i)}=\left(a_{r, 1}^{(i)}, \ldots, a_{r, n}^{(i)}\right)$ by

$$
a_{r, j}^{(i)}= \begin{cases}E_{j}\left(c_{i}\right) & \text { if } j \in \Omega_{r}^{(i)} \cup \cdots \cup \Omega_{r_{i}}^{(i)}, \\ E_{j}\left(c_{i+1}\right) & \text { otherwise. }\end{cases}
$$

We also set $\mathbf{a}_{r_{i}+1}^{(i)}=\mathbf{a}_{1}^{(i+1)}$. Then we form the sequence

$$
\left(\mathbf{a}^{(i)}\right)_{i \geq 0}=\left(\mathbf{a}_{1}^{(0)}, \ldots, \mathbf{a}_{r_{0}}^{(0)}, \mathbf{a}_{1}^{(1)}, \ldots, \mathbf{a}_{r_{1}}^{(1)}, \ldots\right) .
$$

For fixed $i \geq 0$, we note that

$$
E_{j}\left(c_{i+1}\right)=\left\{\begin{array}{ll}
E_{j}\left(c_{i}\right)+1 & \text { if } j \in \Omega^{(i)}, \\
E_{j}\left(c_{i}\right) & \text { otherwise }
\end{array} \quad(1 \leq j \leq n),\right.
$$

because the map $\mathbf{E}$ is constant on $\left[d_{i}, c_{i+1}\right]$ and, by Lemma 7.4, each of its component $E_{j}$ with $j \in \Omega^{(i)}$ admits exactly one point of discontinuity in $\left[c_{i}, d_{i}\right]$ while the other components are constant on $\left[c_{i}, d_{i}\right]$. This means that

$$
\mathbf{a}_{1}^{(i)}=\mathbf{E}\left(c_{i}\right) \quad(i \geq 0)
$$

and that, for each $r=1, \ldots, r_{i}$, the point $\mathbf{a}_{r+1}^{(i)}$ is obtained from $\mathbf{a}_{r}^{(i)}$ by adding 1 to each of its coordinates with index in $\Omega_{r}^{(i)}$. Since these coordinates are consecutive integers and since $\mathbf{a}_{r_{i}+1}^{(i)}=\mathbf{a}_{1}^{(i+1)}$, we conclude that, in general, for each $i \geq 0$, the coordinates of $\mathbf{a}^{(i)}$ with index in $\Omega_{i}$ are consecutive integers and that $\mathbf{a}^{(i+1)}$ is obtained from $\mathbf{a}^{(i)}$ by adding 1 to each of them. As $\Omega_{i}=\left\{k_{i}, \ldots, \ell_{i+1}\right\}$, this yields

$$
\left(a_{1}^{(i)}, \ldots, \widehat{a_{k_{i}}^{(i)}}, \ldots, a_{n}^{(i)}\right)=\left(a_{1}^{(i+1)}, \ldots, \widehat{a_{\ell_{i+1}}^{(i+1)}}, \ldots, a_{n}^{(i+1)}\right) \quad \text { and } \quad a_{\ell_{i+1}}^{(i+1)}=a_{\ell_{i+1}}^{(i)}+1
$$

for each $i \geq 0$.

The triple consisting of $\left(\mathbf{a}^{(i)}\right)_{i \geq 0},\left(k_{i}\right)_{i \geq 0}$ and $\left(\ell_{i}\right)_{i \geq 0}$ is almost a canvas. In view of the above, it satisfies the conditions $(\mathrm{C} 1)$ and (C3) of Definition 1.1 and the slightly weaker condition $1 \leq k_{i} \leq \ell_{i} \leq n(i \geq 0)$ instead of (C2). Nevertheless, we can associate to it a map $\mathbf{R}:\left[t_{0}, \infty\right) \rightarrow \Delta_{n}$ as in Definition [1.2. By construction this map satisfies $\mathbf{R}\left(t_{i}\right)=\mathbf{E}\left(c_{i}\right)$ for each $i \geq 0$ because each point $\mathbf{E}\left(c_{i}\right)$ belongs to the sequence $\left(\mathbf{a}^{(i)}\right)_{i \geq 0}$. Finally, let $\left(i_{m}\right)_{0 \leq m<s}$ denote the sequence of integers $i \geq 0$ with $i=0$ or $k_{i}<\ell_{i}$, listed in increasing order. We leave to the reader to check that the triple $\left(\mathbf{a}^{\left(i_{m}\right)}\right)_{0 \leq m<s},\left(k_{i_{m}}\right)_{0 \leq m<s},\left(\ell_{i_{m}}\right)_{0 \leq m<s}$ is a canvas and that $\mathbf{R}$ is the rigid system attached to it.

Lemma 7.9. With the notation of the preceding lemma, we have $\|\mathbf{R}(t)-\mathbf{P}(t)\|_{\infty} \leq 3 n^{2}$ for each $t \in\left[t_{0}, \infty\right)$. 
Proof. Fix a choice of $t \geq t_{0}$ and let $i \geq 0$ denote the index for which $t \in\left[t_{i}, t_{i+1}\right)$. Using Lemma 7.2 (iii), we find

$$
\left|c_{i}-t\right| \leq\left|c_{i}-t_{i}\right|+t_{i+1}-t_{i}=\left|c_{i}-\sum_{j=1}^{n} E_{j}\left(c_{i}\right)\right|+\sum_{j=1}^{n}\left(E_{j}\left(c_{i+1}\right)-E_{j}\left(c_{i}\right)\right) \leq n(n+1)+n .
$$

As the components of $\mathbf{P}$ are continuous and piecewise linear with slopes 0 and 1 , we deduce that

$$
\left\|\mathbf{P}\left(c_{i}\right)-\mathbf{P}(t)\right\|_{\infty} \leq\left|c_{i}-t\right| \leq n(n+2) .
$$

By Lemma 7.2 (ii), we also have

$$
\left\|\mathbf{R}\left(t_{i}\right)-\mathbf{P}\left(c_{i}\right)\right\|_{\infty}=\left\|\mathbf{E}\left(c_{i}\right)-\mathbf{P}\left(c_{i}\right)\right\|_{\infty} \leq n+1 .
$$

Finally, since the components of $\mathbf{R}$ are monotone increasing on $\left[t_{i}, t_{i+1}\right]$, we find

$$
\left\|\mathbf{R}(t)-\mathbf{R}\left(t_{i}\right)\right\|_{\infty} \leq\left\|\mathbf{R}\left(t_{i+1}\right)-\mathbf{R}\left(t_{i}\right)\right\|_{\infty}=\left\|\mathbf{E}\left(c_{i+1}\right)-\mathbf{E}\left(c_{i}\right)\right\|_{\infty}=1 .
$$

Combining the three preceding displayed inequalities, we conclude that $\|\mathbf{R}(t)-\mathbf{P}(t)\|_{\infty} \leq$ $n(n+2)+(n+1)+1 \leq 3 n^{2}$.

\section{Proof of Theorem 1.3}

Since any rigid $n$-system is an $(n, 0)$-system, the first result below proves the second assertion of our main Theorem 1.3 in a quantitative form.

Theorem 8.1. Let $q_{0} \geq 0$ and let $\mathbf{P}:\left[q_{0}, \infty\right) \rightarrow \mathbb{R}^{n}$ be an $(n, 0)$-system. Then there exists $a$ unit vector $\mathbf{u}$ of $\mathbb{R}^{n}$ such that $\left\|\mathbf{P}(q)-\mathbf{L}_{\mathbf{u}}(q)\right\|_{\infty} \leq 3 n^{2}(n+9)$ for each $q \geq q_{0}$.

Proof. We first note that $\mathbf{P}$ can be extended to an $(n, 0)$-system on $[0, \infty)$ in the following way. Put $t_{0}=0$ and $t_{i}=P_{1}\left(q_{0}\right)+\cdots+P_{i}\left(q_{0}\right)$ for $i=1, \ldots, n$. Then we have $t_{n}=q_{0}$ and we define

$$
\mathbf{P}(q)=\Phi_{n}\left(0, \ldots, 0, P_{1}\left(q_{0}\right), \ldots, P_{i-1}\left(q_{0}\right), q-t_{i-1}\right) \quad\left(t_{i-1} \leq q \leq t_{i}, 1 \leq i \leq n\right),
$$

so that, for $i=1, \ldots, n$ the combined graph of $\mathbf{P}$ over $\left[t_{i-1}, t_{i}\right]$ consists of $n-1$ horizontal line segments, not necessarily distinct, with ordinates $0, \ldots, 0, P_{1}\left(q_{0}\right), \ldots, P_{i-1}\left(q_{0}\right)$ and one line segment of slope 1 joining the points $\left(t_{i-1}, 0\right)$ and $\left(t_{i}, P_{i}\left(q_{0}\right)\right)$.

In view of the above observation, we may assume that $q_{0}=0$. Put $\delta=n+7$ and $\tilde{q}_{0}=n(n+1) \delta / 2$. Since all $(n, 0)$-systems are reduced, Proposition 7.1 shows the existence of a rigid $n$-system $\mathbf{R}:\left[\tilde{q}_{0}, \infty\right) \rightarrow \mathbb{R}^{n}$ of mesh $\delta$ such that $\|\mathbf{P}(q)-\mathbf{R}(q)\|_{\infty} \leq 3 n^{2} \delta$ for each $q \geq \tilde{q}_{0}$. For this rigid system, Theorem 5.5] shows in turn the existence of a unit vector $\mathbf{u}$ in $\mathbb{R}^{n}$ such that $\left\|\mathbf{R}(q)-\mathbf{L}_{\mathbf{u}}(q)\right\|_{\infty} \leq n \log \left(8 e^{4} n\right)$ for each $q \geq \tilde{q}_{0}$. Then, we have

$$
\left\|\mathbf{P}(q)-\mathbf{L}_{\mathbf{u}}(q)\right\|_{\infty} \leq 3 n^{2} \delta+n \log \left(8 e^{4} n\right) \quad\left(q \geq \tilde{q}_{0}\right) .
$$

However, for $q \in\left[0, \tilde{q}_{0}\right]$, the coordinates of $\mathbf{P}(q)$ are non-negative and bounded above by $P_{1}(q)+\cdots+P_{n}(q)=q \leq \tilde{q}_{0}$ while those of $\mathbf{L}_{\mathbf{u}}(q)$ are also non-negative and bounded above by $L_{\mathbf{u}, 1}(q)+\cdots+L_{n, q}(q) \leq \tilde{q}_{0}+n \log (n)$ thanks to (2.2). Thus the estimate (8.1) extends to all $q \geq 0$ and the conclusion follows because $3 n^{2} \delta+n \log \left(8 e^{4} n\right) \leq 3 n^{2}(n+9)$. 
Since any rigid $n$-system of a given mesh $\delta>0$ is also a rigid system of mesh $\delta / N$ for each integer $N \geq 1$, our last result below implies the first assertion of Theorem 1.3, thereby completing the proof of that theorem.

Theorem 8.2. Let $\delta>24 n^{4} 2^{n} \log (n)$ and let $\mathbf{u}$ be a unit vector of $\mathbb{R}^{n}$. Put $q_{0}=n(n+1) \delta / 2$. Then there exists a rigid n-system $\mathbf{R}:\left[q_{0}, \infty\right) \rightarrow \mathbb{R}^{n}$ of mesh $\delta$ such that $\left\|\mathbf{L}_{\mathbf{u}}(q)-\mathbf{R}(q)\right\|_{\infty} \leq$ $4 n^{2} \delta$ for each $q \geq q_{0}$.

Proof. Put $\gamma=6 n 2^{n} \log (n)$. By Theorem 2.9 , there exists an $(n, \gamma)$-system $\mathbf{P}:[0, \infty) \rightarrow \mathbb{R}^{n}$ such that $\left\|\mathbf{L}_{\mathbf{u}}(q)-\mathbf{P}(q)\right\|_{\infty} \leq \gamma$ for each $q \geq 0$. Then, by Proposition 6.2, there exists an $(n, 2 n \gamma)$-reduced system $\tilde{\mathbf{P}}:[0, \infty) \rightarrow \mathbb{R}^{n}$ for which $\|\mathbf{P}(q)-\tilde{\mathbf{P}}(q)\|_{\infty} \leq n \gamma$ for each $q \geq 0$. Finally, since $\delta>4 n^{3} \gamma$, Proposition 7.1 provides a rigid $n$-system $\mathbf{R}:\left[q_{0}, \infty\right) \rightarrow \mathbb{R}^{n}$ with mesh $\delta$ satisfying $\|\tilde{\mathbf{P}}(q)-\mathbf{R}(q)\|_{\infty} \leq 3 n^{2} \delta$ for each $q \geq q_{0}$. Thus, for $q \geq q_{0}$, we get $\left\|\mathbf{L}_{\mathbf{u}}(q)-\mathbf{R}(q)\right\|_{\infty} \leq(n+1) \gamma+3 n^{2} \delta \leq 4 n^{2} \delta$.

\section{REFERENCES}

[1] Y. Bugeaud and M. Laurent, On transfer inequalities in Diophantine approximation II, Math. Z. 265 (2010), 249-262.

[2] O. N. German, Intermediate Diophantine exponents and parametric geometry of numbers, Acta Arith. 154 (2012), 79-101.

[3] P. M. Gruber and C. G. Lekkerkerker, Geometry of numbers, North-Holland, 1987.

[4] V. Jarník, Zum Khintchineschen Übertragungssatz, Trav. Inst. Math. Tbilissi 3 (1938), $193-212$.

[5] A. Y. Khintchine, Zur metrischen Theorie der Diophantischen Approximationen, Math. Z. 24 (1926), $706-714$.

[6] A. Y. Khintchine, Über eine Klasse linearer Diophantischer Approximationen, Rend. Circ. Math. Palermo 50 (1926), 170-195.

[7] M. Laurent, Exponents of Diophantine approximation in dimension two, Can. J. Math. 61 (2009), $165-189$.

[8] M. Laurent, On transfer inequalities in Diophantine Approximation, in: Analytic Number Theory in Honour of Klaus Roth, Cambridge U. Press (2009), 306-314.

[9] K. Mahler, On compound convex bodies I, II, Proc. Lond. Math. Soc. 5 (1955), 358-384.

[10] N. G. Moshchevitin, Exponents for three-dimensional simultaneous Diophantine approximations, Czechoslovak Math. J. 62 (2012), 127-137.

[11] D. Roy, Construction of points realizing the regular systems of Wolfgang Schmidt and Leonard Summerer, J. Théor. Nombres Bordeaux, to appear.

[12] W. M. Schmidt, On heights of algebraic subspaces and diophantine approximations, Ann. of Math. 85 (1967), 430-472.

[13] W. M. Schmidt and L. Summerer, Parametric geometry of numbers and applications, Acta Arith. 140 (2009), 67-91.

[14] W. M. Schmidt and L. Summerer, Diophantine approximation and parametric geometry of numbers, Monatsh. Math. 169 (2013), 51-104.

[15] W. M. Schmidt and L. Summerer, Simultaneous approximation to three numbers, Mosc. J. Comb. Number Theory 3 (2013), 84-107.

Département de Mathématiques, Université d’Ottawa, 585 King Edward, Ottawa, Ontario K1N 6N5, CANADA

E-mail address: droy@uottawa.ca 